\title{
Record statistics and persistence for a random walk with a drift
}

\author{
Satya N. Majumdar ${ }^{1}$, Grégory Schehr ${ }^{1}$ and Gregor Wergen ${ }^{2}$ \\ ${ }^{1}$ Laboratoire de Physique Théorique et Modèles Statistiques, UMR 8626, Université \\ Paris Sud 11 and CNRS, Bât. 100, Orsay F-91405, France \\ ${ }^{2}$ Institut für Theoretische Physik, Universität zu Köln, 50937 Köln, Germany \\ E-mail: \\ satya.majumdar@u-psud.fr,gregory.schehr@u-psud.fr, gw@thp.uni-koeln.de
}

\begin{abstract}
.
We study the statistics of records of a one-dimensional random walk of $n$ steps, starting from the origin, and in presence of a constant bias $c$. At each time-step the walker makes a random jump of length $\eta$ drawn from a continuous distribution $f(\eta)$ which is symmetric around a constant drift $c$. We focus in particular on the case were $f(\eta)$ is a symmetric stable law with a Lévy index $0<\mu \leq 2$. The record statistics depends crucially on the persistence probability which, as we show here, exhibits different behaviors depending on the sign of $c$ and the value of the parameter $\mu$. Hence, in the limit of a large number of steps $n$, the record statistics is sensitive to these parameters $(c$ and $\mu$ ) of the jump distribution. We compute the asymptotic mean record number $\left\langle R_{n}\right\rangle$ after $n$ steps as well as its full distribution $P(R, n)$. We also compute the statistics of the ages of the longest and the shortest lasting record. Our exact computations show the existence of five distinct regions in the $(c, 0<\mu \leq 2)$ strip where these quantities display qualitatively different behaviors. We also present numerical simulation results that verify our analytical predictions.
\end{abstract}

PACS numbers: 02.50.Ga, 05.40.Fb, 05.45.Tp 


\section{Introduction}

The statistical properties of record-breaking events in stochastic processes have been a popular subject of research in recent years. The theory of records has found many interesting applications. Record events are very important in sports [1, 2] and climatology [3, 4, 5, 6], but have also been found relevant in biology [7], in the theory of spin-glasses [8, 9] and in models of growing networks [10]. Also in finance, recordbreaking events, e.g., when the price of a stock breaks its previous records, can lead to increased financial activities [11, 12]. In all of these fields researchers have recently made progress in understanding and modeling the statistics of records by comparing the records in observational data with various kinds of stochastic processes. In this context it has become increasingly important to improve our understanding of the record statistics of elementary stochastic processes. In this paper we focus on one such elementary stochastic process namely a random walk in presence of a constant bias. We show that even for such a simple process, its record statistics is considerably nontrivial and rich.

In general, one is interested in the record events of a discrete-time series of random variables (RV's) $x_{0}, x_{1}, \ldots, x_{n}$. An (upper) record is an entry $x_{k}$, which exceeds all previous entries: $x_{k}>\max \left(x_{0}, x_{1}, \ldots, x_{k-1}\right)$. Until the end of the last century record statistics was fully understood only in the case when the entries of the time series are independent and identically distributed (i.i.d.) RV's (see for instance [13, 14, 15]). For i.i.d. RV's from a continuous distribution $p(x)$ the probability $r_{n}$ of a record in the $n$-th time step is given by 13 ]

$$
r_{n}:=\operatorname{Prob}\left[x_{n}>\max \left(x_{0}, x_{1}, \ldots, x_{n-1}\right)\right]=\frac{1}{n+1},
$$

which is universal, i.e., independent of the parent distribution $p(x)$. This universality follows simply from the isotropy in ordering, i.e., any one of the $(n+1)$ entries is equally probable to be a record. Let $R_{n}$ denote the total number of records up to step $n$. The mean record number is then simply $\left\langle R_{n}\right\rangle=\sum_{m=0}^{n} r_{m}$, which grows asymptotically as $\sim \ln n$ for large $n$.

Due to the numerous applications of the theory of records it became interesting to consider more general models. There has been a lot of interest in the record statistics of RV's which are uncorrelated but not identical anymore. For instance Ballerini et al. considered uncorrelated RV's with a linear drift [16]. More recently Franke et al. studied the same problem as well and found numerous new results [17, 18, 19] by also considering the correlations between individual record events. This model was then successfully applied to the statistics of temperature records in the context of global warming [5]. In 2006 Krug studied the statistics of records of uncorrelated RV's with a time-increasing standard deviation, a model with important biological implications [20].

Another important issue is the study of record statistics for correlated random variables. For weak correlations, with a finite correlation time, one would expect that the record statistics for a large sequence to be asymptotically similar to the uncorrelated case. This is no longer true when there are strong correlations between the entries. 
Perhaps, one of the simplest and most natural time series with strong correlations between its entries corresponds to the positions of a one dimensional random walk [21]. Despite the striking importance and abundance of random walk in various areas of research, the record statistics of a single, discrete-time random walk with a symmetric jump distribution was not computed and understood until only a few years ago. In 2008, Majumdar and Ziff [22] computed exactly the record statistics of a one dimensional symmetric random walk model and showed that the record rate of such a process is completely universal for any continuous and symmetric jump distribution, thanks to the so called Sparre Andersen theorem [23. They considered a time series of RV's $x_{m}$ given by:

$$
x_{m}=x_{m-1}+\eta_{m},
$$

where $\eta_{m}$ 's are i.i.d. RV's drawn from a symmetric and continuous jump distribution $f(\eta)$ (it includes even Lévy flights where $f(\eta) \sim 1 /|\eta|^{\mu+1}$ with $0<\mu<2$ ). Then, the record rate $r_{n}$ for such a process is given by the universal formula [22]

$$
r_{n}=\left(\begin{array}{c}
2 n \\
n
\end{array}\right) 2^{-2 n} \stackrel{n \rightarrow \infty}{\longrightarrow} \frac{1}{\sqrt{\pi n}},
$$

independently of the jump distribution $f(\eta)$. They also computed exactly the mean record number $\left\langle R_{n}\right\rangle$ and even its full distribution [22. In addition, there exists nice connection between the record statistics and the extreme value statistics for the one dimensional symmetric jump processes and many universal results can be subsequently derived using the Sparre Andersen theorem (see 24] for a review).

Following Ref. [22], there has been considerable interests in generalising them to more general set of strongly correlated stochastic processes. For instance, Sabhapandit discussed symmetric random walks with a random, possibly heavy tailed, waiting time between the individual jumps (the so called Continuous Time Random Walk model) 25]. Recently the present authors considered the record statistics of an ensemble of $N$ independent and symmetric random walks [12. There, in contrast to the case of a single random walker, the record statistics of $N$ Lévy flights with a heavy-tailed jump distribution was found to be different from the one of $N$ Gaussian random walkers with a jump distribution that has a finite second moment.

Another important generalization is to consider a single one dimensional random walker but with asymmetric jump distribution, for instance, in presence of a constant bias $c$. First steps towards this generalization were taken by Le Doussal and Wiese in 2009 [26] who derived the exact record statistics for a biased random walker with a Cauchy jump distribution (a special case of Lévy flights with Lévy index $\mu=1$ ). More recently in 2011, Wergen et al. showed that a biased random walk is useful to model record-breaking events in daily stock prices [11]. They were able to obtain results in some special limits of a biased random walker with a Gaussian jump distribution. Apart from these two special cases, namely the Cauchy and the Gaussian jump distribution, there are no other analytical results available, to our knowledge, for other jump distributions for a biased random walker. Recently, the record statistics for a biased random walker 
was also studied numerically in order to quantify the contamination spread in a porous medium via the particle tracking simulations [27].

In this article we present a complete analysis of the record statistics for a biased random walker with arbitrary jump distributions. As we will see, the record statistics depends crucially on the persistence probability $Q(n)$ [see Eq. (17) below], the probability that the biased walker stays to the left of its initial starting position up to $n$ steps. While persistence probability for various stochastic processes have been extensively studied in the recent past [28], it seems that for this simple biased jump process, it has not been systematically studied in the literature to the best of our knowledge. Here we provide exact results for the persistence probability $Q(n)$ for a biased random walk arbitrary jump distributions [see Eq. (67)], which subsequently leads to the exact record statistics for the same process.

The rest of the paper is organized as follows. Since the paper is long with many detailed results, we provide in section 2 a short review on the record statistics for random walks both with and without bias, followed by a summary of the main results of this paper. Readers not interested in the details of the calculations can skip the rest of the paper. In section 3, we will show how to use the renewal property of the random walk and a generalized version of the Sparre Andersen theorem [23] to compute the persistence of random walks in presence of both positive and negative drift. The results for the persistence are interesting on their own and will be discussed in detail in section 4 , but they will also allow us to compute the record statistics. In particular we will show that, in the presence of drift, the complete universality found for the record statistics in the unbiased case [22] breaks down and there are five different types of asymptotic behaviors which emerge depending on the two parameters of the model, namely the drift $c$ and the index $0<\mu \leq 2$ characterizing the tail of the jump distribution. This record statistics will be discussed in detail in section [5, Later, in section 6, we will also discuss the extreme value statistics of the ages of the longest (section 6.1) and the shortest lasting records (section 6.2) in each of the regimes. We will show that the asymptotic behavior of these quantities is also systematically different in the five regimes. Finally in section 7 , we will conclude with some open problems.

\section{Record statistics for random walks: A short review and a summary of new results}

In this section, we provide a short review on the record statistics of a one dimensional random walk model, with and without external drift. This will also serve to set up our notations for the rest of the paper. At the end of this section, we summarize the main new results obtained in this work.

Let us first start with the driftless case. Consider a sequence of random variables

$\left\{x_{0}=0, x_{1}, x_{2}, \ldots, x_{n}\right\}$ where $x_{m}$ represents the position of a discrete-time unbiased random walker at step $m$. The walker starts at the origin and its position evolves via the Markov rule $x_{m}=x_{m-1}+\eta_{m}$, where $\eta_{m}$ represents the stochastic jump at the 
$m$-th step. The jump variables $\eta_{m}$ 's are i.i.d. random variables, each drawn from the common probability distribution function (pdf) $f(\eta)$, normalized to unity. The pdf $f(\eta)$ is continuous and symmetric with zero mean. Let $\hat{f}(k)=\int_{-\infty}^{\infty} f(\eta) e^{i k \eta} d \eta$ denote the Fourier transform of the jump distribution. We will henceforth focus on jump distributions $f(\eta)$ whose Fourier transform has the following small $k$ behavior

$$
\hat{f}(k)=1-\left(l_{\mu}|k|\right)^{\mu}+\ldots
$$

where $0<\mu \leq 2$ and $l_{\mu}$ represents a typical length scale associated with the jump. The exponent $0<\mu \leq 2$ dictates the large $|\eta|$ tail of $f(\eta)$. For jump densities with a finite second moment $\sigma^{2}=\int_{-\infty}^{\infty} \eta^{2} f(\eta) d \eta$, such as Gaussian, exponential, uniform etc, one evidently has $\mu=2$ and $l_{2}=\sigma / \sqrt{2}$. In contrast, $0<\mu<2$ corresponds to jump densities with fat tails $f(\eta) \sim|\eta|^{-1-\mu}$ as $|\eta| \rightarrow \infty$. A typical example is $\hat{f}(k)=\exp \left[-|k|^{\mu}\right]$ where $\mu=2$ corresponds to the Gaussian jump distribution, while $0<\mu<2$ corresponds to Lévy flights (for reviews on these jump processes see [29, 30]).

A quantity that will play a crucial role later is $P_{n}(x)$ which denotes the probability density of the position of the symmetric random walk at step $n$. Using the Markov rule in Eq. (2), it is easy to see that $P_{n}(x)$ satisfies the recursion relation

$$
P_{n}(x)=\int_{-\infty}^{\infty} P_{n-1}\left(x^{\prime}\right) f\left(x-x^{\prime}\right) d x^{\prime},
$$

starting from $P_{0}(x)=\delta(x)$. This recurrence relation can be trivially solved by taking Fourier transform and using the convolution structure. Inverting the Fourier transform, one gets

$$
P_{n}(x)=\int_{-\infty}^{\infty} \frac{d k}{2 \pi}[\hat{f}(k)]^{n} e^{-i k x}
$$

In the limit of large $n$, the small $k$ behavior of $\hat{f}(k)$ dominates the integral on the right hand side (rhs) of Eq. (6). Substituting the small $k$ behavior from Eq. (4), one easily finds that for $0<\mu<2$, typically $x \sim l_{\mu} n^{1 / \mu}$ and $P_{n}(x)$ approaches the scaling form [29]

$$
P_{n}(x) \rightarrow \frac{1}{l_{\mu} n^{1 / \mu}} \mathcal{L}_{\mu}\left(\frac{x}{l_{\mu} n^{1 / \mu}}\right), \quad \text { where } \quad \mathcal{L}_{\mu}(y)=\int_{-\infty}^{\infty} \frac{d k}{2 \pi} e^{-|k|^{\mu}} e^{-i k y} .
$$

For $0<\mu<2$, the scaling function $\mathcal{L}_{\mu}(y)$ decays as a power law for large $|y|[29]$

$$
\mathcal{L}_{\mu}(y) \underset{y \rightarrow \infty}{\longrightarrow} \frac{A_{\mu}}{|y|^{\mu+1}}, \quad \text { where } A_{\mu}=\frac{1}{\pi} \sin (\mu \pi / 2) \Gamma(1+\mu)
$$

In particular, for $\mu=1$, the scaling function $\mathcal{L}_{1}(y)$ is precisely the Cauchy density itself

$$
\mathcal{L}_{1}(y)=\frac{1}{\pi} \frac{1}{1+y^{2}}
$$

In contrast, for $\mu=2$, the central limit theorem holds, $x \sim \sigma n^{1 / 2}$, and $P_{n}(x)$ approaches a Gaussian scaling form

$$
P_{n}(x) \rightarrow \frac{1}{\sigma n^{1 / 2}} \mathcal{L}_{2}\left(\frac{x}{\sigma n^{1 / 2}}\right), \quad \text { where } \quad \mathcal{L}_{2}(y)=\frac{1}{\sqrt{2 \pi}} \exp \left(-y^{2} / 2\right)
$$


From the sequence of symmetric random variables representing the position of a discrete-time unbiased random walker, we next construct a new sequence of random variables $\left\{y_{0}=0, y_{1}, y_{2}, \ldots, y_{n}\right\}$ where

$$
y_{m}=x_{m}+c m \text { implying } y_{m}=y_{m-1}+c+\eta_{m},
$$

where $\eta_{m}$ 's are symmetric i.i.d. jump variables each drawn from the pdf $f(\eta)$. Clearly, $y_{m}$ then represents the position of a discrete-time random walker at step $m$ in presence of a constant bias $c$.

In this paper, we are interested in the record statistics of this biased sequence $\left\{y_{0}=\right.$ $\left.0, y_{1}, y_{2}, \ldots, y_{n}\right\}$. A record happens at step $m$ if $y_{m}>\max \left(y_{0}=0, y_{1}, y_{2}, \ldots, y_{m-1}\right)$, i.e., if the position of the biased walker $y_{m}$ at step $m$ is bigger than all previous positions, with the convention that the initial position $y_{0}=0$ is counted as a record. Let $R_{n}$ denote the number of records up to step $n$. Clearly, $R_{n}$ is a random variable and we denote its distribution by

$$
P(R, n)=\text { Proba. }\left[R_{n}=R\right] .
$$

We would like to compute the asymptotic properties of this record number distribution $P(R, n)$ for large $n$, for arbitrary drift $c$ and for arbitrary symmetric and continuous jump density $f(\eta)$ whose Fourier transform $\hat{f}(k)$ has the small $k$ behavior as in Eq. (4) with the index $0<\mu \leq 2$.

In absence of a drift, i.e., for $c=0$, the distribution $P(R, n)$ was computed exactly in Ref. [22] for all $R$ and $n$, using a renewal property of the record process. Amazingly, the distribution was found to be completely universal, i.e., independent of the jump distribution $f(\eta)$ (as long as it is symmetric and continuous) for all $R$ and $n$ 22. In particular, for large $n$, it was shown that $P(R, n)$ has a scaling form [22]

$$
P(R, n) \approx \frac{1}{\sqrt{n}} g_{0}\left(\frac{R}{\sqrt{n}}\right),
$$

where the universal scaling function

$$
g_{0}(x)=\frac{1}{\sqrt{\pi}} \exp \left(-x^{2} / 4\right), \text { for } x \geq 0
$$

is a half-Gaussian. Consequently, the mean and the variance of the number of records grows asymptotically as 22]

$$
\left\langle R_{n}\right\rangle \approx \frac{2}{\sqrt{\pi}} n^{1 / 2}, \quad\left\langle R_{n}^{2}\right\rangle-\left\langle R_{n}\right\rangle^{2} \approx 2\left(1-\frac{2}{\pi}\right) n .
$$

The renewal property of the record process derived originally for the unbiased random walker in Ref. 22] was then generalized to the case with a nonzero drift $c$ in Ref. [26]. In particular, the authors of Ref. [26] studied in detail the special case of the Cauchy jump distribution $f_{\text {Cauchy }}(\eta)=1 /\left[\pi\left(1+\eta^{2}\right)\right]$ [which belongs to the $\mu=1$ 
family of jump densities in Eq. (44)] and found that the mean number of records $\left\langle R_{n}\right\rangle$ grows algebraically with $n$ for large $n$ with an exponent that depends continuously on $c[26]$

$$
\left\langle R_{n}\right\rangle \approx \frac{1}{\Gamma(1+\theta(c))} n^{\theta(c)}, \quad \text { where } \quad \theta(c)=\frac{1}{2}+\frac{1}{\pi} \arctan (c) .
$$

In addition, the asymptotic distribution $P(R, n)$ for large $n$ was found [26] to have a scaling distribution, $P(R, n) \sim n^{-\theta(c)} g_{c}\left(R n^{-\theta(c)}\right)$ with a nontrivial scaling function $g_{c}(x)$ which reduces, for $c=0$, to the half-Gaussian in Eq. (14).

For jump densities with a finite second moment $\sigma^{2}$ and in presence of a nonzero positive drift $c>0$, the mean number of records $\left\langle R_{n}\right\rangle$ was analysed in Ref. [1] and was found to grow linearly with $n$ for large $n,\left\langle R_{n}\right\rangle \approx a_{2}(c) n$ where the prefactor $a_{2}(c)$ was computed approximately for the Gaussian jump distribution. However, an exact expression of the prefactor for arbitrary jump densities with a finite $\sigma^{2}$ is missing. In addition, these results were then applied [11] to analyse the record statistics of stock prices from the Standard and Poors 500. The distribution of the record number $P(R, n)$ for large $n$ has not been studied for jump densities with a finite second moment.

In this paper, we present detailed exact results for the asymptotic record number distribution $P(R, n)$ for large $n$, for arbitrary drift $c$ (both positive and negative) and for arbitrary symmetric and continuous jump densities $f(\eta)$ with Fourier transform $\hat{f}(k)$ having a small $k$ behavior as in Eq. (4) parametrized by the exponent $0<\mu \leq 2$. We find a variety of rather rich behaviors for $P(R, n)$ depending on the value of $c$ and the exponent $\mu$. On the strip $(c, 0<\mu \leq 2)$ (see Fig. 1), we find five distinct regimes: (I) when $0<\mu<1$ with $c$ arbitrary (II) when $\mu=1$ and $c$ arbitrary (III) when $1<\mu<2$ and $c>0(\mathrm{IV})$ when $\mu=2$ and $c>0$ and $(\mathrm{V})$ when $1<\mu \leq 2$ and $c<0$. In these five regimes the record statistics behave differently, resulting in different asymptotic forms for the record number distribution $P(R, n)$. The line $\mu=1$ (regime II above) is a critical line on which the record statistics exhibits marginal behavior. These five regimes are summarized in the phase diagram in the $(c, 0<\mu \leq 2)$ strip in Fig. 1].

As we will see later, a quantity that plays a crucial role in the study of record statistics is the persistence $Q(n)$ which denotes the probability that the process $y_{m}$ in Eq. (11) stays below its initial value $y_{0}$ up to step $n$, i.e.,

$$
Q(n)=\text { Proba. }\left[y_{i}<y_{0} \text {, for all } i=1,2, \ldots, n\right] \text {. }
$$

Due to the translational invariance of the process, $Q(n)$ does not depend on $y_{0}$. The persistence probability has been studied quite extensively in recent years in a variety of theoretical and experimental systems [28]. We will see that even for the simple stochastic process $y_{m}$ representing the position of a discrete-time random walker in presence of a drift, the persistence $Q(n)$ has a rather rich asymptotic behavior depending on the parameters $\mu$ and $c$. Hence, even though here our main interest is in the record statistics, we include the results for the persistence $Q(n)$ as a byproduct.

We also analyse the statistics of waiting times between individual record events. In particular we are interested in the expected ages of the longest and the shortest lasting 


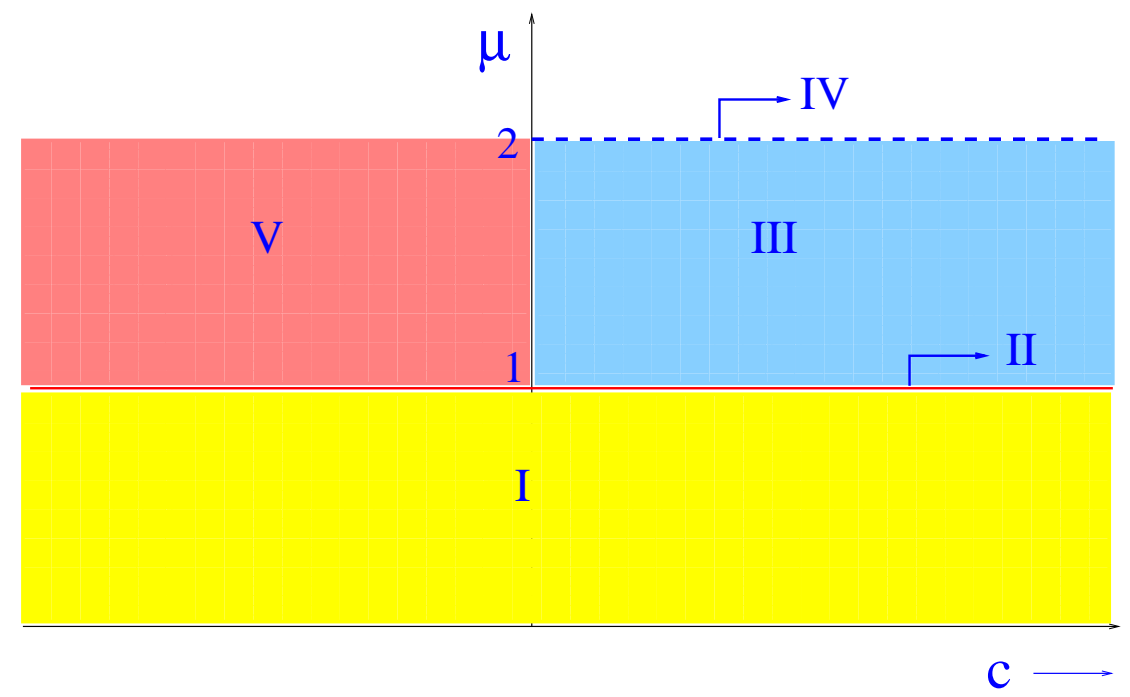

Figure 1. Phase diagram in the $(c, 0<\mu \leq 2)$ strip depicting 5 regimes: (I) $0<\mu<1$ and $c$ arbitrary (II) the line $\mu=1$ and $c$ arbitrary (III) $1<\mu<2$ and $c>0$ (IV) the semi-infinite line $\mu=2$ and $c>0$ and (V) $1<\mu \leq 2$ and $c<0$. The persistence $Q(n)$, the record number distribution $P(R, n)$ and the mean ages of the longest and the shortest lasting record exhibit different asymptotic behaviors in these 5 regimes (see text).

records. The age of the longest lasting record is defined as:

$$
l_{\max , n}=\max \left(l_{1}, l_{2}, \ldots, l_{R}\right),
$$

where $l_{i}$ is the length of the time interval between the $i$-th and the $(i+1)$-th record. Similarly one defines the age of the shortest lasting record as

$$
l_{\min , n}=\min \left(l_{1}, l_{2}, \ldots, l_{R}\right) .
$$

In [22], the mean values of $l_{\max , n}$ and $l_{\min , n}$ were computed exactly for the symmetric random walk with arbitrary jump distribution. It was found that [22] for large $n$

$$
\left\langle l_{\max , \mathrm{n}}\right\rangle \sim C_{0} n,
$$

where $C_{0} \approx 0.626508 \ldots$ is a universal constant independent of the jump distribution. Interestingly, the same constant $C_{0}$ also appears in other related problems [33, 34]. In contrast, the shortest record exhibits different behavior for large $n$ [22]

$$
\left\langle l_{\min , \mathrm{n}}\right\rangle \sim \sqrt{n / \pi} .
$$

In this paper we generalize these results to the case of a biased random walk and as in the case of record number distribution, we find five different asymptotic behaviors depending on $c$ and $\mu$. 
Summary of the new results: Let us then summarize the main new results in this paper for the asymptotic behavior of the persistence $Q(n)$, the record number distribution $P(R, n)$ and the extremal ages of records in the 5 regimes in the $(c, \mu)$ strip mentioned above.

Regime I $(0<\mu<1$ and $c$ arbitrary): In this regime, we find that the persistence $Q(n)$ decays algebraically for large $n$

$$
Q(n) \approx \frac{B_{I}}{\sqrt{n}}
$$

where the prefactor $B_{I}$ depends on the details of the jump distribution $f(\eta)$ and the drift $c$ and can be computed explicitly [see Eq. (78) ]. The mean record number up to $n$ steps grows asymptotically for large $n$ as

$$
\left\langle R_{n}\right\rangle \approx A_{\mathrm{I}} \sqrt{n}
$$

While the growth exponent $1 / 2$ is universal, i.e. independent of $c$ and the precise form of the jump distribution $f(\eta)$, the prefactor $A_{\mathrm{I}}$ depends on $c$ and on the details of the density $f(\eta)$. In addition, the two prefactors $A_{\mathrm{I}}$ and $B_{\mathrm{I}}$ are related simply via $B_{I}=2 /\left(\pi A_{\mathrm{I}}\right)$. We find the following exact expression for the prefactor $A_{\mathrm{I}}$

$$
A_{\mathrm{I}}=\frac{2}{\sqrt{\pi}} \exp \left[\frac{1}{\pi} \int_{0}^{\infty} \frac{d k}{k} \arctan \left(\frac{\hat{f}(k) \sin (k c)}{1-\hat{f}(k) \cos (k c)}\right)\right] .
$$

In the scaling limit when $n \rightarrow \infty$ and $R \rightarrow \infty$, but with the ratio $R / \sqrt{n}$ fixed, we find that the distribution $P(R, n)$ approaches the scaling form

$$
P(R, n) \approx \frac{2}{A_{\mathrm{I}} \sqrt{\pi n}} g_{0}\left(\frac{2 R}{A_{\mathrm{I}} \sqrt{\pi n}}\right), \quad \text { where } \quad g_{0}(x)=\frac{1}{\sqrt{\pi}} \exp \left(-x^{2} / 4\right) .
$$

Averaging over $R$ evidently reproduces the result in Eq. (23). Thus, the record number, rescaled by the nonuniversal scale factor $R \rightarrow R / A_{\mathrm{I}}$, approaches asymptotically the same universal half-Gaussian scaling distribution as in the driftless case $c=0$ in Eq. (14).

The statistics of the longest lasting record is completely unaffected by the drift $c$ in this regime. For the mean value $\left\langle l_{\max , n}\right\rangle$ we find that

$$
\left\langle l_{\max , n}\right\rangle \sim C_{\mathrm{I}} n
$$

where the same constant $C_{I}=C_{0} \approx 0.626508 \ldots$ was also found in the unbiased case [see Eq. (20)]. The age of the shortest lasting record is given by

$$
\left\langle l_{\min , n}\right\rangle \sim D_{\mathrm{I}} \sqrt{n}
$$

with a prefactor $D_{\mathrm{I}}=B_{\mathrm{I}}$. Therefore, in contrast to $\left\langle l_{\max , n}\right\rangle,\left\langle l_{\min , n}\right\rangle$ slightly differs from the unbiased case and has a prefactor that depends non-trivially on $c$. 
Regime II (the line $\mu=1$ and $c$ arbitrary): On this line, we find that the persistence $Q(n)$ decays algebraically for large $n$ but with an exponent that depends continuously on $c$

$$
Q(n) \approx \frac{B_{\mathrm{II}}}{n^{\theta(c)}}
$$

where the exponent $0 \leq \theta(c) \leq 1$ is given in Eq. (16). In this sense the behavior is marginal. The prefactor $B_{\mathrm{II}}$ can be computed exactly [see Eq. (85)]. The mean record number also grows marginally for large $n$

$$
\left\langle R_{n}\right\rangle \approx \frac{A_{\mathrm{II}}}{\Gamma[1+\theta(c)]} n^{\theta(c)},
$$

where the prefactor $A_{\mathrm{II}}=1 /\left[\Gamma[1-\theta(c)] B_{\mathrm{II}}\right]$. The record number distribution exhibits an asymptotic scaling form

$$
P(R, n) \approx \frac{1}{A_{\mathrm{II}} n^{\theta(c)}} g_{c}\left(\frac{R}{A_{\mathrm{II}} n^{\theta(c)}}\right)
$$

where one can obtain a formal exact expression (109) and explicit tails of the scaling function $g_{c}(x)$ which also exhibits marginal behavior, i.e., depends continuously on $c$.

Like in regime $\mathrm{I}$ we find that the mean age of the longest lasting record grows linearly in $n$, but this time with a non-trivial $c$ dependent prefactor. We find that

$$
\left\langle l_{\max , n}\right\rangle \sim C_{\mathrm{II}} n
$$

where $C_{\mathrm{II}}$ is given in Eq. (147). The mean age of the shortest lasting record is more strongly affected by the drift. Here we find that $\left\langle l_{\text {min, } n}\right\rangle$ grows algebraically with $n$ with an exponent which depends continuously on $c$ :

$$
\left\langle l_{\min , n}\right\rangle \sim D_{\mathrm{II}} n^{1-\theta(c)}
$$

with $D_{\mathrm{II}}=B_{\mathrm{II}}$ as in Eq. (28) and $\theta(c)$ as defined in Eq. (16).

Regime III $(1<\mu<2$ and $c>0)$ : In this regime, the persistence $Q(n)$ decays for large $n$ as

$$
Q(n) \approx \frac{B_{\mathrm{III}}}{n^{\mu}}
$$

where the prefactor $B_{\text {III }}$ depends on the details of the jump distribution and can be computed [see Eq. (90)]. The mean number of records grows linearly with increasing $n$

$$
\left\langle R_{n}\right\rangle \approx a_{\mu}(c) n
$$

where the prefactor $a_{\mu}(c)$ can be computed explicitly [see Eq. (115)]. The record number distribution $P(R, n)$, for large $n$, behaves as

$$
P(R, n) \approx \frac{1}{a_{\mu}(c) n^{1 / \mu}} V_{\mu}\left(\frac{R-a_{\mu}(c) n}{a_{\mu}(c) n^{1 / \mu}}\right)
$$


where the scaling function $V_{\mu}(u)$ can be computed exactly and it has a non-Gaussian form with highly asymmetric tails

$$
\begin{aligned}
V_{\mu}(u) & \approx A_{\mu}|u|^{-\mu-1} \quad \text { as } \quad u \rightarrow-\infty \\
& \approx c_{1} u^{(2-\mu) / 2(\mu-1)} \exp \left[-c_{2} u^{\mu /(\mu-1)}\right] \quad \text { as } \quad u \rightarrow \infty,
\end{aligned}
$$

where $A_{\mu}$ is the same constant as in Eq. (8) and the constants $c_{1}$ and $c_{2}$ are given explicitly by

$$
\begin{aligned}
& c_{1}=\left[2 \pi(\mu-1)\left(\mu B_{\mu}\right)^{1 /(\mu-1)}\right]^{-1 / 2}, \\
& c_{2}=(1-1 / \mu)\left(\mu B_{\mu}\right)^{-1 /(\mu-1)},
\end{aligned}
$$

where

$$
B_{\mu}=-\frac{1}{2 \cos (\mu \pi / 2)}>0 \quad \text { for } \quad 1<\mu<2 .
$$

Thus, in this regime, while the mean record number grows linearly with $n$, the fluctuations around the mean are anomalous $\sim n^{1 / \mu}$ and described by a non-Gaussian distribution.

Also the extremal ages of records have an interesting behavior in this regime. In particular we find that the average age of the longest lasting record grows like

$$
\left\langle l_{\max , n}\right\rangle \sim C_{\mathrm{III}} n^{\frac{1}{\mu}},
$$

where the constant $C_{\mathrm{III}}$ can be computed explicitly [see Eq. (150)]. On the other hand and in contrast to the results of regime I and II, the mean age of the shortest lasting record converges to a finite value:

$$
\left\langle l_{\min , n}\right\rangle \sim D_{\mathrm{III}}=1-a_{\mu}(c),
$$

which thus depends continuously on $c$. The strongly different $n$ dependence of $\left\langle l_{\max , n}\right\rangle$ and $\left\langle l_{\min , n}\right\rangle$ in the regime I and in the regime III is a consequence of the fact that while in regime I the asymptotic behavior is dominated by the fluctuations, in regime III the effect of the drift is stronger in the large $n$ limit.

Regime IV ( the semi-infinite line $\mu=2$ and $c>0$ ): On this semi-infinite line the variance $\sigma^{2}$ of the jump pdf is finite. This leads to an exponential tail of the persistence $Q(n)$ for large $n$. More precisely we show that

$$
Q(n) \approx \frac{B_{\mathrm{IV}}}{n^{3 / 2}} \exp \left[-\left(c^{2} / 2 \sigma^{2}\right) n\right],
$$

where the nonuniversal prefactor $B_{\mathrm{IV}}$ can be computed exactly [see Eq. (96)]. We also show that the mean and the variance of the record number both grow linearly for large $n$

$$
\left\langle R_{n}\right\rangle \approx a_{2}(c) n \text { and }\left\langle R_{n}^{2}\right\rangle-\left\langle R_{n}\right\rangle^{2} \approx b_{2}(c) n,
$$

where the amplitudes $a_{2}(c)$ and $b_{2}(c)$ are nonuniversal and depend on the details of the jump distribution $f(\eta)$. We provide exact expressions for these amplitudes respectively 
in Eqs. (125) and (128) as well as in Appendix B. The distribution of the record number $P(R, n)$ approaches a Gaussian form asymptotically for large $n$

$$
P(R, n) \approx \frac{1}{\sqrt{2 \pi b_{2}(c) n}} \exp \left[-\frac{1}{2 b_{2}(c) n}\left(R-a_{2}(c) n\right)^{2}\right] .
$$

Thus, in this regime, the mean record number grows linearly with $n$ with normal Gaussian fluctuations $\sim n^{1 / 2}$ around the mean.

It is interesting to see that the asymptotic behavior of $\left\langle l_{\max , n}\right\rangle$ in regime IV is qualitatively different from regime III. Here we find that $\left\langle l_{\max , n}\right\rangle$ grows only logarithmically with $n$ for $n \rightarrow \infty$ :

$$
\left\langle l_{\max , n}\right\rangle \sim C_{\mathrm{IV}} \ln n
$$

with an $n$ independent constant $C_{\mathrm{IV}}=\frac{2 \sigma^{2}}{c^{2}}$. Like in regime III, the average age of the shortest lasting record approaches a (different) constant value depending on $c$ :

$$
\left\langle l_{\min , n}\right\rangle \sim D_{\mathrm{IV}}=1-a_{2}(c)
$$

which depends continuously on $c$.

Regime V $(1<\mu \leq 2$ and $c<0)$ : In this case, the walker predominantly moves towards the negative axis due to the drift. Consequently, the events where the walker crosses the origin from the negative to the positive side become extremely rare. As a result, with a finite probability the walker stays forever on the negative side. Thus, the persistence $Q(n)$ approaches a constant for large $n$

$$
Q(n) \rightarrow \alpha_{\mu}(c)
$$

Similarly, the occurrence of the records (with positive record values) are also rare. Subsequently, we find that the mean record number also approaches a constant for large $n$

$$
\left\langle R_{n}\right\rangle \rightarrow \frac{1}{\alpha_{\mu}(c)}
$$

where the constant $\alpha_{\mu}(c)$ is given by

$$
\begin{aligned}
\alpha_{\mu}(c) & =a_{\mu}(|c|) \quad \text { for } \quad 1<\mu<2, \\
& =a_{2}(|c|) \quad \text { for } \quad \mu=2,
\end{aligned}
$$

where $a_{\mu}(c)$ and $a_{2}(c)$ are precisely the amplitude of the linear growth of the mean record number respectively in regime III and IV [respectively in Eqs. (34) and (44)]. An explicit expression for $\alpha_{\mu}(c)$ is given in Eq. (101). The record number distribution $P(R, n)$ also approaches a steady state, i.e., $n$-independent distribution as $n \rightarrow \infty$. This distribution has a purely geometric form

$$
P(R, n \rightarrow \infty)=\alpha_{\mu}(c)\left[1-\alpha_{\mu}(c)\right]^{R-1} .
$$


Physically this result is easy to understand because for $c<0$ and $\mu>1$, the walker typically moves away from the origin on the negative side with very rare and occasional excursions to the positive side caused by rare large jumps. As a result, the occurrence of a record is like a Poisson process which eventually leads to a geometric distribution as in Eq. (52).

In this regime the statistics of the longest and the shortest lasting records are particularly simple. Since the record number is finite, the longest lasting record will grow linearly in $n$ :

$$
\left\langle l_{\max , n}\right\rangle \sim C_{\mathrm{V}} n, C_{\mathrm{V}}=1 .
$$

For the shortest lasting record we find a similar behavior:

$$
\left\langle l_{\min , n}\right\rangle \sim \alpha_{\mu}(c) n,
$$

with the same $c$ dependent constant $\alpha_{\mu}(c)$ as in Eq. (48). Here, the main contributions to these quantities come from trajectories that never cross the origin and stay negative for all $n$.

The five regimes in the $(c, 0<\mu \leq 2)$ strip are depicted in Fig. 1. As mentioned above, the line $\mu=1$ is a special 'critical' line with marginal exponents that depend continuously on the drift $c$. It is not difficult to understand physically why $\mu=1$ plays a special role. Indeed, writing $y_{n}=x_{n}+c n$ where $x_{n}$ represents a symmetric random walk, we see that the two terms $x_{n}$ and $c n$ compete with each other for large $n$. Since $x_{n} \sim n^{1 / \mu}$ for $0<\mu \leq 2$ [see Eq. (7)], it is clear that for $0<\mu<1$, the term $x_{n}$ dominates over the drift and the presence of a nonzero drift only leads to subleading asymptotic effect. In contrast, for $\mu>1$, the drift term starts to play an important role in governing the asymptotic record statistics. In the region $1<\mu<2$ and $c>0$ (regime III), while the mean record number increases linearly with $n$ due to the dominance of the drift term, the typical fluctuation around the mean is still dominated by the $x_{n} \sim n^{1 / \mu}$ term [see Eq. (35)]. However when $\mu=2$ and $c>0$ (regime IV), the drift term completely dominates over the $x_{n}$ term leading to Gaussian fluctuations around the mean. This competition between $x_{n}$ and $c n$ thus leads to (i) a 'phase transition' in the asymptotic behavior of record statistics of $y_{n}$ at the critical value $\mu=1$ and (ii) an anomalous region with non-Gaussian fluctuations around the mean in the region $1<\mu<2$ and $c>0$.

\section{Record Number distribution via renewal property and the generalized Sparre Andersen theorem}

The idea of using the renewal property of random walks to compute the distribution of record number was first used in Ref. [22] for symmetric random walks and was subsequently generalized to biased random walks [26]. We briefly summarize below the main idea.

Consider the random sequence $\left\{y_{0}, y_{1}, y_{2}, \ldots,\right\}$ representing the successive positions

of a discrete-time biased random walker evolving via Eq. (11), starting from an arbitrary 
initial position $y_{0}$. Consider first the persistence $Q(n)$ defined in Eq. (17). Let us also define

$$
F(n)=\text { Proba. }\left[y_{1}<y_{0}, y_{2}<y_{0}, \ldots, y_{n-1}<y_{0}, y_{n}>y_{0}\right]
$$

which denotes the probability that the walker crosses its initial position $y_{0}$ from below for the first time at step $n$. Clearly

$$
F(n)=Q(n-1)-Q(n) .
$$

It is also useful to define the generating functions

$$
\tilde{Q}(z)=\sum_{n=0}^{\infty} Q(n) z^{n}, \quad \tilde{F}(z)=\sum_{n=1}^{\infty} F(n) z^{n} .
$$

Using the relation in Eq. (56) it follows that

$$
\tilde{F}(z)=1-(1-z) \tilde{Q}(z) .
$$

Consider now any realization of the sequence $\left\{y_{0}=0, y_{1}, y_{2}, \ldots, y_{n}\right\}$ up to $n$ steps and let $R_{n}$ be the number of records in this realization. Let $\vec{l}=\left\{l_{1}, l_{2}, \ldots, l_{R}\right\}$ denote the time intervals between successive records in this sequence (see Fig. 2). Clearly $l_{i}$ denotes the age of the $i$-th record, i.e., the time up to which the $i$-th record survives. The last record, i.e. the $R$-th record, stays a record till step $n$. Let $P(\vec{l}, R \mid n)$ denote the joint distribution of the ages and the number of records up to step $n$. Using the two probabilities $Q(n)$ and $F(n)$ defined earlier and the fact that the successive intervals between records are statistically independent due to the Markov nature of the process, it follows immediately that

$$
P(\vec{l}, R \mid n)=F\left(l_{1}\right) F\left(l_{2}\right) \ldots F\left(l_{R}\right) Q\left(l_{R}\right) \delta_{\sum_{i=1}^{R} l_{i}, N},
$$

where the Kronecker delta enforces the global constraint that the sum of the time intervals equals $n$. The fact that the last record, i.e. the $R$-th record, is still surviving as a record at step $n$ indicates that the distribution $Q\left(l_{R}\right)$ of $l_{R}$ is different from the preceding ones. It is easy to check that $P(\vec{l}, R \mid n)$ is normalized to unity when summed over $\vec{l}$ and $R$. The record number distribution $P(R, n)=\sum_{\vec{l}} P(\vec{l}, R \mid n)$ is just the marginal of the joint distribution when one sums over the interval lengths. Due to the presence of the delta function, this sum is most easily carried out by considering the generating function with respect to $n$. Multiplying Eq. (59) by $z^{n}$ and summing over $\vec{l}$ and $n$, one arrives at the fundamental relation

$$
\sum_{n=0}^{\infty} P(R, n) z^{n}=[\tilde{F}(z)]^{R-1} \tilde{Q}(z)=[1-(1-z) \tilde{Q}(z)]^{R-1} \tilde{Q}(z)
$$

where we used the relation in Eq. (58). Note that, by definition, $R \leq(n+1)$, i.e. $P(R, n)=0$ if $n<R-1$. Hence, the sum in Eq. (60) actually runs from $n=R-1$ to $\infty$. 


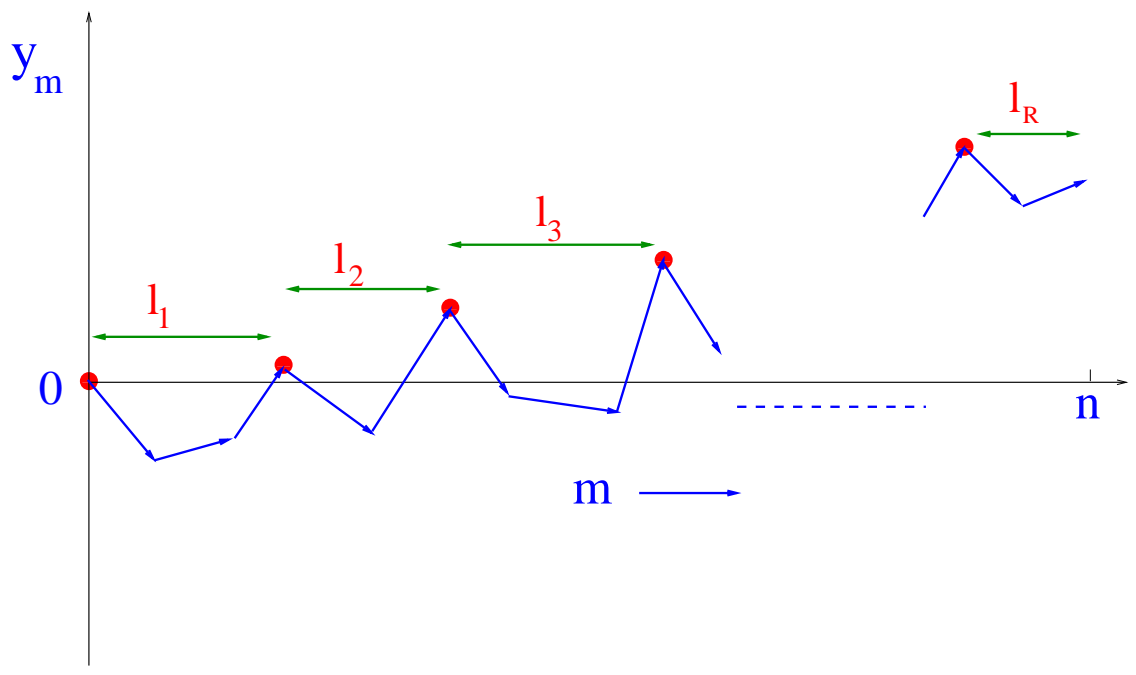

Figure 2. A typical realization of the biased random walk sequence $\left\{y_{0}=\right.$ $\left.0, y_{1}, y_{2}, \ldots, y_{n}\right\}$ of $n$ steps with $R$ records. Each record is represented by a filled circle. The set $\left\{l_{1}, l_{2}, \ldots, l_{R-1}\right\}$ represents the time intervals between the successive records and $l_{R}$ is the age of the last record which is still a record till step $n$.

Thus the basic object is the generating function $\tilde{Q}(z)$. Once this is determined, one can, at least in principle, compute other quantities such as the statistics of records or their ages using the fundamental renewal equation (60). Fortunately, there exists a beautiful combinatorial identity first derived by Sparre Andersen [23] that allows one to compute $\tilde{Q}(z)$

$$
\tilde{Q}(z)=\sum_{n=0}^{\infty} Q(n) z^{n}=\exp \left[\sum_{n=1}^{\infty} \frac{z^{n}}{n} p(n)\right],
$$

where $p(n)=$ Proba. $\left[y_{n}<0\right]$. Using the relation $y_{n}=x_{n}+c n$ where $x_{n}$ represents the symmetric random walk at step $n$ in Eq. (21) one gets, $p(n)=$ Proba. $\left[x_{n}<-c n\right]$. Then, using the pdf $P_{n}(x)$ of the symmetric walk $x_{n}$ at step $n$ in Eq. (6) , one gets

$$
p(n)=\text { Proba. }\left[x_{n}<-c n\right]=\int_{-\infty}^{-c n} P_{n}(x) d x=\int_{c n}^{\infty} P_{n}(x) d x,
$$

where, in obtaining the last equality we used the symmetry $P_{n}(x)=P_{n}(-x)$. Substituting this expression of $p(n)$ in Eq. (61) gives

$$
\tilde{Q}(z)=\sum_{n=0}^{\infty} Q(n) z^{n}=\exp \left[\sum_{n=1}^{\infty} \frac{z^{n}}{n} \int_{c n}^{\infty} P_{n}(x) d x\right] .
$$

Eq. (63), with $P_{n}(x)$ given by Eq. (6), determines $\tilde{Q}(z)$ in terms of the Fourier transform $\hat{f}(k)$ of the jump distribution $f(\eta)$. Subsequently Eq. (60) then determines, in principle, the record number distribution $P(R, n)$. In the driftless case $c=0$, great simplification occurs, since by symmetry $\int_{0}^{\infty} P_{n}(x) d x=1 / 2$. This gives, from Eq. (63), 
$\tilde{Q}(z)=1 / \sqrt{1-z}$. This is completely universal as all the dependence on the jump distribution $f(\eta)$ drops out. Subsequently, Eq. (60) provides, for $c=0$, the universal result for the record number distribution [22]

$$
\sum_{n=0}^{\infty} P(R, n) z^{n}=\frac{(1-\sqrt{1-z})^{R-1}}{\sqrt{1-z}},
$$

which, when inverted, yields 22] for large $n$ the scaling behavior in Eq. (13) with the scaling function given by the half-Gaussian form in Eq. (14).

However, in presence of a nonzero bias $c$, extraction of the precise large $n$ behavior of $P(R, n)$ from the set of equations (60), (63) and (61) is more complicated. For the special case of the Cauchy distribution, this was performed in Ref. [26] which showed nontrivial behavior. The rest of this paper is devoted precisely to this technical task of extracting the large $n$ behavior of $P(R, n)$ for a general jump distribution $f(\eta)$ and we will see that a variety of rather rich asymptotic behavior emerges depending on the value of the drift $c$ and the exponent $\mu$ characterizing the small $k$ behavior of $\hat{f}(k)$ in Eq. (44).

Before finishing this section, let us remark that from Eq. (60) one can also compute the generating functions of the moments of the number of records. For example, multiplying Eq. (60) by $R$, summing over $R$ and using the identity $\sum_{n=0}^{\infty} n x^{n-1}=$ $1 /(1-x)^{2}$ we get for the first moment

$$
\sum_{n=0}^{\infty}\left\langle R_{n}\right\rangle z^{n}=\frac{1}{(1-z)^{2} \tilde{Q}(z)} .
$$

Similarly, multiplying Eq. (60) by $R^{2}$ and summing over $R$ one gets for the second moment

$$
\sum_{n=0}^{\infty}\left\langle R_{n}^{2}\right\rangle z^{n}=\frac{2-(1-z) \tilde{Q}(z)}{(1-z)^{3} \tilde{Q}^{2}(z)} .
$$

We will use these two results later in Section IVB.

\section{Asymptotic behavior of persistence $Q(n)$ for large $n$}

The persistence $Q(n)$, i.e. the probability that the process $y_{n}$ stays below its initial value $y_{0}$ up to step $n$ and its generating function $\tilde{Q}(z)$ is the key ingredient to determine the record number distribution $P(R, n)$ via Eq. (60). Apart from its key role as an input for the record statistics, the persistence $Q(n)$ for this process is, by itself, an interesting quantity to study. We will see in this section that even for the simple stochastic process $y_{n}$, representing the position of a discrete-time random walker in presence of a drift, the persistence $Q(n)$ has a rather rich asymptotic behavior depending on the parameters $\mu$ and $c$. Before getting into the details of the derivation, it is useful to summarize these asymptotic results. We find that for large $n$, the persistence $Q(n)$ has the following 
asymptotic tails depending on $\mu>0$ and $c$

$$
\begin{aligned}
& Q(n) \sim B_{\mathrm{I}} n^{-1 / 2} \quad \text { for } \quad 0<\mu<1 \text { and } c \text { arbitrary (regime I), } \\
& \sim B_{\mathrm{II}} n^{-\theta(c)} \quad \text { for } \quad \mu=1 \text { and } c \text { arbitrary (regime II), } \\
& \sim B_{\text {III }} n^{-\mu} \text { for } 1<\mu<2 \text { and } c>0 \text { (regime III), } \\
& \sim B_{\mathrm{IV}} n^{-3 / 2} \exp \left[-\left(c^{2} / 2 \sigma^{2}\right) n\right] \text { for } \quad \mu=2 \text { and } c>0 \text { (regime IV), } \\
& \sim \alpha_{\mu}(c) \text { for } 1<\mu \leq 2 \text { and } c<0 \quad \text { (regime } \mathrm{V} \text { ), }
\end{aligned}
$$

where the prefactors $B_{\mathrm{I}}, B_{\mathrm{II}}, B_{\mathrm{III}}, B_{\mathrm{IV}}$ can be explicitly computed. In regime $\mathrm{V}, \alpha_{\mu}(c)$ is a constant independent of $n$ that can also be computed explicitly [see Eq. (101) and Appendix B for $\alpha_{2}(\mu)$ ]. The exponent $\theta(c)$ depends continuously on $c$ and is given in Eq. (16) [see also Eq. (80)]. In Fig. 3 these results are confirmed numerically for the regimes I-IV.

To derive these asymptotic behaviors of $Q(n)$ for large $n$, we start with the key result in Eq. (63). Using Cauchy's inversion formula in the complex $z$ plane one can write

$$
Q(n)=\int_{C_{0}} \frac{d z}{2 \pi i} \frac{1}{z^{n+1}} \tilde{Q}(z) \quad \text { with } \quad \tilde{Q}(z)=\exp \left[\sum_{n=1}^{\infty} \frac{z^{n}}{n} \int_{c n}^{\infty} P_{n}(x) d x\right],
$$

where the contour $C_{0}$ encircles the origin 0 and is free of any singularity of $\tilde{Q}(z)$ (see Fig. (4). Let $z^{*}$ denote the singularity of $\tilde{Q}(z)$ on the real axis closest to the origin. Then, one can deform the contour $C_{0}$ to $C_{1}$ (see Fig. (4) such that the vertical part of $C_{1}$ is located just left of $z^{*}$ and the circular part has radius $r_{1}$. By taking the $r_{1} \rightarrow \infty$ limit, it follows from Eq. (68) that for large $n$, the contribution from the circular part vanishes exponentially. Thus for large $n$, the leading contribution comes from the vertical part of $C_{1}$, i.e the imaginary axis located just left of $z^{*}$. Next we make a change of variable $z=e^{-s n}$ and define

$$
\begin{gathered}
\tilde{q}(s)=\tilde{Q}\left(z=e^{-s}\right)=\sum_{n=0}^{\infty} Q(n) e^{-s n}=\exp \left[W_{c, \mu}(s)\right], \\
\text { where } \quad W_{c, \mu}(s)=\sum_{n=1}^{\infty} \frac{e^{-s n}}{n} \int_{c n}^{\infty} P_{n}(x) d x .
\end{gathered}
$$

Using this expression in the integrand in Eq. (68) and retaining only the contribution from the vertical part of the contour $C_{1}$ for large $n$, we get

$$
Q(n) \approx \int_{s^{*}-i \infty}^{s^{*}+i \infty} \frac{d s}{2 \pi i} e^{s n} \exp \left[W_{c, \mu}(s)\right]
$$

where $W_{c, \mu}(s)$ is given in Eq. (70) and $s^{*}=-\ln \left(z^{*}\right)$ is the singularity of $\tilde{q}(s)=$ $\exp \left[W_{c, \mu}(s)\right]$ on the real axis closest to $s=0$. Identifying the integral on the rhs of Eq. (71) as a standard Bromwich integral in the complex $s$ plane, we see that for large $n, Q(n)$ is essentially given by the inverse Laplace transform of the function 


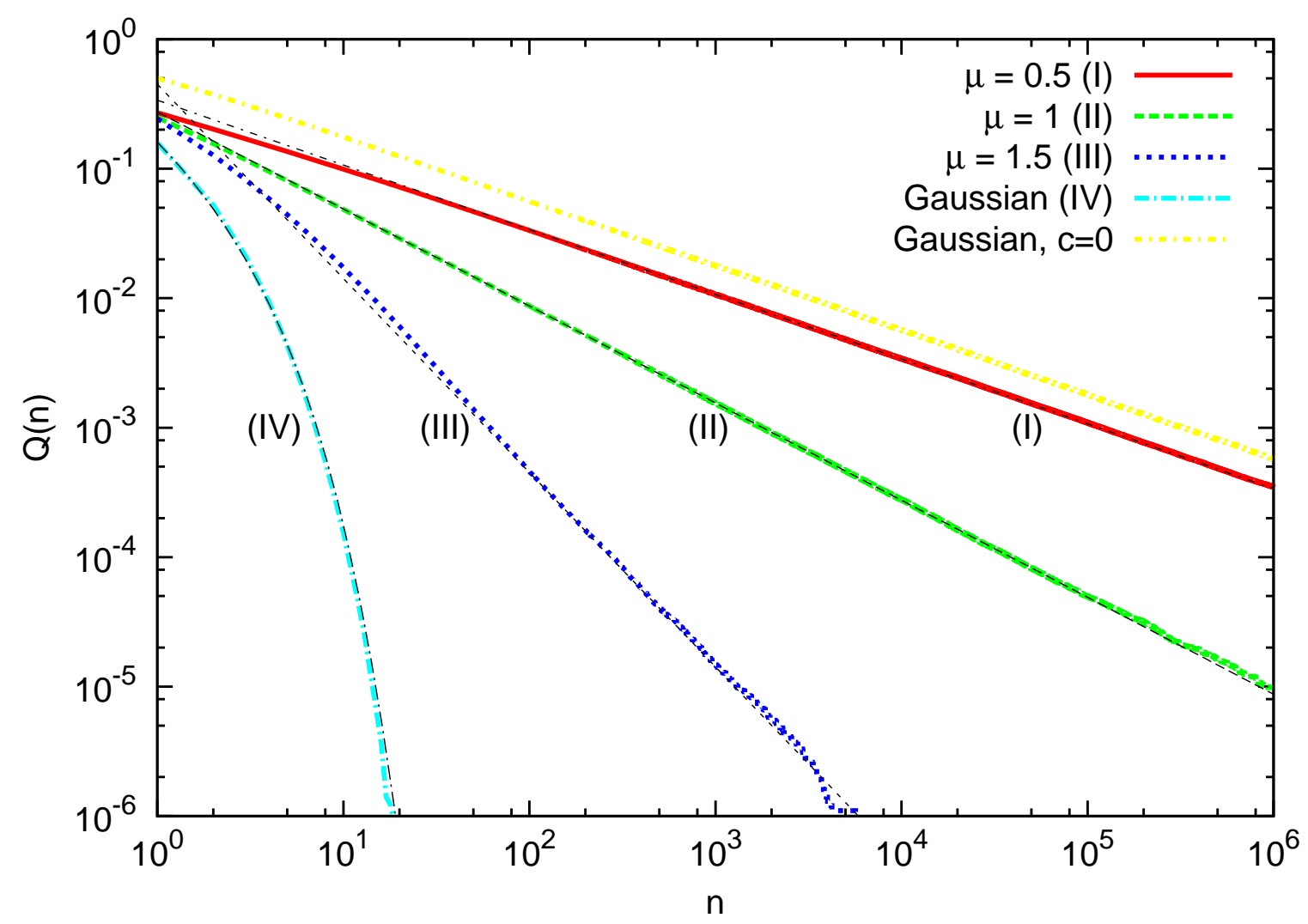

Figure 3. Numerical simulations of the persistence $Q(n)$, i.e. the probability that a random walker with a bias $c$ stays below its initial position up to step $n$. We considered 4 different Lévy-stable jump distributions characterized respectively by the Lévy index $\mu=0.5,1,1.5$ and $\mu=2$ (in the last case it is just Gaussian jump distribution). In all cases, we had a constant positive bias $c=1$ and the data were obtained by averaging over $10^{7}$ samples. For comparison, we also present the result for the unbiased case $(c=0)$ with a Gaussian jump distribution (the top curve). The thin dashed lines give our analytical predictions from Eq. (67) with fitted prefactors $B_{\mathrm{I}}, B_{\mathrm{II}}, B_{\mathrm{III}}$ and $B_{\mathrm{IV}}$. For the $\mu=1$ case we used $\theta(c=1) \approx 0.7498 \ldots$.

$\tilde{q}(s)=\exp \left[W_{c, \mu}(s)\right]$. To make further progress, we need to first identify the position of the singularity $s^{*}$ of $W_{c, \mu}(s)$ and then analyse the dominant contribution in the Bromwich integral coming from the neighborhood of $s^{*}$ for large $n$. We see below that the singular behavior of $W_{c, \mu}(s)$ as a function of $s$ depends on the parameters $c$ and $\mu>0$ and there are essentially 5 regimes in the $(c, 0<\mu \leq 2)$ strip as shown in Fig. 1. Below we discuss these regimes separately.

\subsection{Regime I: $0<\mu<1$ and c arbitrary}

To analyse the leading singularity of $W_{c, \mu}(s)$ as a function of $s$ in this regime, it is first convenient to use the normalization condition $\int_{-\infty}^{\infty} P_{n}(x) d x=1$ and the symmetry $P_{n}(x)=P_{n}(-x)$ to rewrite

$$
\int_{c n}^{\infty} P_{n}(x) d x=\frac{1}{2}-\int_{0}^{c n} P_{n}(x) d x .
$$




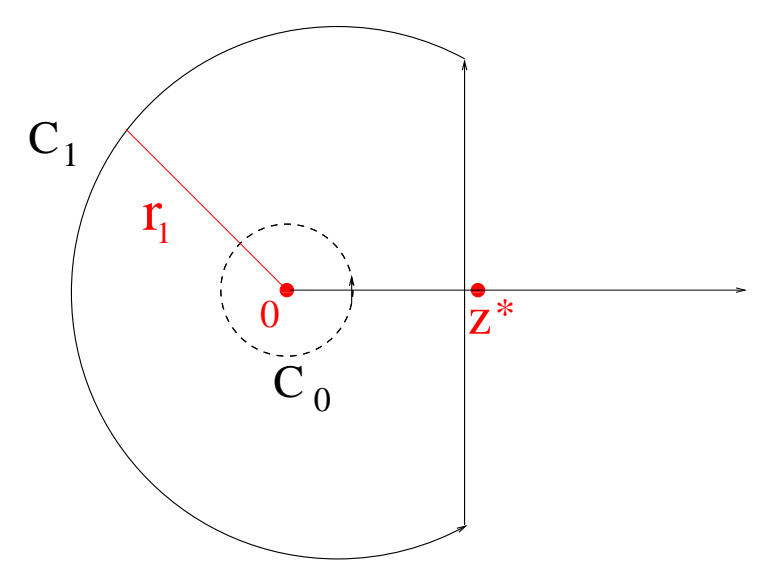

Figure 4. The contour $C_{0}$ in the complex $z$ plane can be deformed to the contour $C_{1}$. In the large $n$ limit, the dominant contribution to the Cauchy integral in Eq. (68) comes from the vertical part of $C_{1}$.

Substituting this in Eq. (70) gives

$$
W_{c, \mu}(s)=-\frac{1}{2} \ln \left(1-e^{-s}\right)-\sum_{n=1}^{\infty} \frac{e^{-s n}}{n} \int_{0}^{c n} P_{n}(x) d x .
$$

Now, as $s \rightarrow 0$, the sum in Eq. (173) converges to a constant for $0<\mu<1$

$$
S_{0}=\sum_{n=1}^{\infty} \frac{1}{n} \int_{0}^{c n} P_{n}(x) d x .
$$

To see this, let us see how the integral $\int_{0}^{c n} P_{n}(x) d x$ behaves for large $n$. For large $n$, we can use the scaling form for $P_{n}(x)$ in Eq. (17). One finds that $\int_{0}^{c n} P_{n}(x) d x \rightarrow$ $\int_{0}^{c n^{(1-1 / \mu)}} \mathcal{L}_{\mu}(y) d y$ as $n \rightarrow \infty$. For $0<\mu<1$, clearly this integral decreases leading to the convergence of the series in Eq. (174). Thus, the leading singularity of $W_{c, \mu}(s)$ occurs near $s=s^{*}=0$ where it behaves as

$$
W_{c, \mu}(s) \approx-\frac{1}{2} \ln (s)-S_{0}
$$

Substituting this result in Eq. (70) gives

$$
\tilde{q}(s)=\sum_{n=0}^{\infty} Q(n) e^{-s n} \underset{s \rightarrow 0}{\longrightarrow} \frac{e^{-S_{0}}}{\sqrt{s}} .
$$

We now substitute this singular behavior of the integrand in Eq. (171) after setting $s^{*}=0$ and perform the standard Bromwich integral (one can use the fact that the inverse Laplace transform $L T_{s \rightarrow n}^{-1}\left[s^{-1 / 2}\right]=1 / \sqrt{\pi n}$ )

$$
Q(n) \underset{n \rightarrow \infty}{\longrightarrow} \frac{B_{\mathrm{I}}}{\sqrt{n}}
$$


where the prefactor $B_{\mathrm{I}}$ is given by

$$
B_{\mathrm{I}}=\frac{e^{-S_{0}}}{\sqrt{\pi}}=\frac{1}{\sqrt{\pi}} \exp \left[-\sum_{n=1}^{\infty} \frac{1}{n} \int_{0}^{c n} P_{n}(x) d x\right] .
$$

\subsection{Regime II: $\mu=1$ and c arbitrary}

The case $\mu=1$ is rather special and marginal as we demonstrate now. Consider the sum $W_{c, 1}(s)$ in Eq. (70). In this case, it follows from Eq. (17) that $P_{n}(x) \rightarrow(1 / n) \mathcal{L}_{1}(x / n)$ as $n \rightarrow \infty$, where $\mathcal{L}_{1}(y)=1 /\left[\pi\left(1+y^{2}\right)\right]$ for all $y$ and hence is integrable. Thus the integral $\int_{c n}^{\infty} P_{n}(x) d x$ converges to a constant for large $n$

$$
\int_{c n}^{\infty} P_{n}(x) d x \underset{n \rightarrow \infty}{\longrightarrow} \int_{c}^{\infty} \mathcal{L}_{1}(y) d y=1-\theta(c),
$$

where

$$
\theta(c)=\int_{-\infty}^{c} \mathcal{L}_{1}(y) d y=\frac{1}{2}+\frac{1}{\pi} \arctan (c) .
$$

Hence, the $n$-th term of the sum in $W_{c, 1}(s)$ behaves, for large $n$, as $T_{n} \rightarrow(1-\theta(c)) e^{-s n} / n$. Consequently, the sum $W_{c, 1}(s)=\sum_{n \geq 1} T_{n}$ has a singularity at $s=s^{*}=0$. The leading asymptotic behavior near this singularity reads

$$
W_{c, 1}(s) \underset{s \rightarrow 0}{\longrightarrow}-(1-\theta(c)) \ln (s)-\gamma_{0}
$$

where $\gamma_{0}$ is a constant that depends on the details of $P_{n}(x)$, in particular on the difference between $P_{n}(x)$ and its large $n$ asymptotic form $(1 / n) \mathcal{L}_{1}(x / n)$ for finite $n$

$$
\gamma_{0}=\sum_{n=1}^{\infty}\left[1-\theta(c)-\int_{c n}^{\infty} P_{n}(x) d x\right] \text {. }
$$

Using this result on the right hand side (rhs) of Eq. (170) gives

$$
\tilde{q}(s)=\underset{s \rightarrow 0}{\longrightarrow} \frac{e^{-\gamma_{0}}}{s^{1-\theta(c)}} .
$$

Substituting this result in the Bromwich contour in Eq. (71) (after setting $s^{*}=0$ ) and performing the Bromwich integral gives

$$
Q(n) \underset{n \rightarrow \infty}{\longrightarrow} \frac{B_{\mathrm{II}}}{n^{\theta(c)}}
$$

where

$$
B_{\mathrm{II}}=\frac{e^{-\gamma_{0}}}{\Gamma[1-\theta(c)]} \quad \text { and } \quad \theta(c)=\frac{1}{2}+\frac{1}{\pi} \arctan (c),
$$

and $\gamma_{0}$ in given in Eq. (82).

Thus, for $\mu=1$, the persistence $Q(n)$ decays algebraically for large $n$ but with an exponent $\theta(c)$ that depends continuously on the drift $c$. In this sense the line $\mu=1$ is 
marginally critical. The exponent $\theta(c)$ in Eq. (85) increases continuously with $c$ from $\theta(c \rightarrow-\infty)=0$ to $\theta(c \rightarrow \infty)=1$.

Let us end this subsection with the following remark on the special case of pure Cauchy jump distribution, $f_{\text {Cauchy }}(\eta)=1 /\left[\pi\left(1+\eta^{2}\right)\right]$. As mentioned before, the record statistics for this case was studied in detail in Ref. [26]. For the Cauchy case, it is well known that $P_{n}(x)=(1 / n) f_{\text {Cauchy }}(x / n)=(1 / n) \mathcal{L}_{1}(x / n)$ for all $n$. As a result, it follows from Eq. (82) that the constant $\gamma_{0}=0$ in this case. However, in the general $\mu=1$ case (not necessarily the Cauchy case), $\gamma_{0}$ is generically nonzero. Thus, while the persistence exponent $\theta(c)=1 / 2+\frac{1}{\pi} \arctan (c)$ is universal for all jump densities belonging to the $\mu=1$ case, the amplitude $B_{\mathrm{II}}$ is nonuniversal and depends on the details of the jump density.

\subsection{Regime III: $1<\mu<2$ and $c>0$}

To analyse the singular behavior of the sum $W_{c, \mu}(s)$ in Eq. (170) in this regime, we consider the $n$-th term of the sum $T_{n}=\left(e^{-s n} / n\right) \int_{c n}^{\infty} P_{n}(x) d x$ and substitute, for large $n$, the scaling behavior of $P_{n}(x)$ in Eq. (7). This gives $T_{n} \approx\left(e^{-s n} / n\right) \int_{c n^{(1-1 / \mu)}}^{\infty} \mathcal{L}_{\mu}(y) d y$. For $1<\mu<2$, the lower limit of the integral in $T_{n}$ becomes large as $n \rightarrow \infty$ and we can use the tail behavior in Eq. (88) to estimate, $T_{n} \approx\left(A_{\mu} / \mu c^{\mu}\right) e^{-s n} / n^{\mu}$ for large $n$. Hence the sum, $W_{c, \mu}(s)=\sum_{n=1}^{\infty} T_{n}$ clearly converges to a constant $W_{c, \mu}(0)$ as $s \rightarrow 0$. For small $s$, one can replace the sum by an integral and estimate exactly the first singular correction to this constant. This gives

$$
W_{c, \mu}(s) \underset{s \rightarrow 0}{\longrightarrow} W_{c, \mu}(0)-B_{\mu} s^{\mu-1},
$$

where the constant $B_{\mu}=A_{\mu} \Gamma(2-\mu) /\left[\mu(\mu-1) c^{\mu}\right]$. Using the exact expression of $A_{\mu}$ from Eq. (8) and simplify, one finds $B_{\mu}=-1 /[2 \cos (\mu \pi / 2)]>0$ as in Eq. (40). Note also that from the definition in (70)

$$
\tilde{q}(0)=\exp \left[W_{c, \mu}(0)\right]=\exp \left[\sum_{n=1}^{\infty} \frac{1}{n} \int_{c n}^{\infty} P_{n}(x) d x\right] .
$$

Substituting the small $s$ behavior from Eq. (86) in Eq. (70) gives

$$
\tilde{q}(s) \underset{s \rightarrow 0}{\longrightarrow} \tilde{q}(0)\left[1-B_{\mu} s^{\mu-1}+\ldots\right] .
$$

Substituting this singular behavior of $\tilde{q}(s)=\exp \left[W_{c, \mu}(s)\right]$ in the Bromwich integral in Eq. (71) (upon setting $s^{*}=0$ ) and performing the integral by standard method provides the following large $n$ power law tail for $Q(n)$

$$
Q(n) \underset{n \rightarrow \infty}{\longrightarrow} \frac{B_{\mathrm{III}}}{n^{\mu}},
$$

where the prefactor $B_{\mathrm{III}}$ is given by

$$
B_{\mathrm{III}}=\frac{(\mu-1) B_{\mu}}{\Gamma(2-\mu) c^{\mu}} \tilde{q}(0)=-\frac{(\mu-1)}{2 \cos (\mu \pi / 2) \Gamma(2-\mu) c^{\mu}} \exp \left[\sum_{n=1}^{\infty} \frac{1}{n} \int_{c n}^{\infty} P_{n}(x) d x\right] .
$$




\subsection{Regime $I V: \mu=2$ and $c>0$}

In this regime, the leading singularity $s^{*}$ of $W_{c, \mu}(s)$ occurs not at $s=0$, but at $s=s^{*}=-s_{1}$ where $s_{1}=c^{2} / 2 \sigma^{2}$. To see this, let us again consider the $n$-th term of the sum $W_{c, \mu}(s)$, i.e. $T_{n}=\left(e^{-s n} / n\right) \int_{c n}^{\infty} P_{n}(x) d x$. For large $n, P_{n}(x)$ now has the Gaussian scaling form in Eq. (10) due to the central limit theorem. Substituting this Gaussian form and carrying out the integration one gets,

$$
T_{n} \rightarrow \frac{e^{-s n}}{2 n} \operatorname{erfc}\left(\frac{c}{\sigma \sqrt{2}} \sqrt{n}\right), \quad \text { where } \operatorname{erfc}(y)=\frac{2}{\sqrt{\pi}} \int_{y}^{\infty} e^{-x^{2}} d x .
$$

Using the asymptotic behavior $\operatorname{erfc}(y) \approx e^{-y^{2}} / y \sqrt{\pi}$ for large $y$, one finds that

$$
T_{n} \underset{n \rightarrow \infty}{\longrightarrow} \frac{\sigma}{c \sqrt{2 \pi}} \frac{e^{-\left(s+s_{1}\right) n}}{n^{3 / 2}}, \quad \text { where } \quad s_{1}=\frac{c^{2}}{2 \sigma^{2}} .
$$

Consequently, the sum $W_{c, \mu}(s)=\sum_{n \geq 1} T_{n}$ actually, while perfectly analytic near $s=0$, has a singularity near $s=s^{*}=-s_{1}$. Close to this singular value, by taking the limit $s+c^{2} / 2 \sigma^{2} \rightarrow 0$ whereby replacing the sum by an integral over $n$, one finds the following leading singular behavior of $W_{c, \mu}(s)$ near $s=-s_{1}$

$$
W_{c, \mu}(s) \underset{s \rightarrow-s_{1}}{\longrightarrow} W_{c, \mu}\left(-s_{1}\right)-\sqrt{2} \frac{\sigma}{c} \sqrt{s+s_{1}},
$$

where $W_{c, \mu}\left(-s_{1}\right)$ is just a constant. Substituting this leading singular behavior on the rhs of Eq. (70) gives

$$
\tilde{q}(s) \underset{s \rightarrow 0}{\longrightarrow} e^{W_{c, \mu}\left(-s_{1}\right)}\left[1-\sqrt{2} \frac{\sigma}{c} \sqrt{s+s_{1}}+\ldots\right] .
$$

We set $s^{*}=-s_{1}$ in the Bromwich contour in Eq. (71), substitute the singular behavior of $\tilde{q}(s)$ in Eq. (94) and perform the Bromwich integral to get

$$
Q(n) \underset{n \rightarrow \infty}{\longrightarrow} \frac{B_{\mathrm{IV}}}{n^{3 / 2}} e^{-s_{1} n} \quad \text { where } \quad s_{1}=\frac{c^{2}}{2 \sigma^{2}}
$$

and the prefactor

$$
B_{\mathrm{IV}}=\frac{\sigma e^{W_{c, \mu}\left(-s_{1}\right)}}{c \sqrt{2 \pi}}=\frac{\sigma}{c \sqrt{2 \pi}} \exp \left[\frac{e^{s_{1} n}}{n} \int_{c n}^{\infty} P_{n}(x) d x\right] .
$$

Thus, contrary to regimes I, II and III, here the persistence $Q(n)$ has a leading exponential tail (modulated by a power law $n^{-3 / 2}$ ).

\subsection{Regime $V: 1<\mu \leq 2$ and $c<0$}

In this regime $c=-|c|<0$ and $\mu>1$. It is convenient, using the normalization condition $\int_{-\infty}^{\infty} P_{n}(x) d x=1$, to first reexpress the sum $W_{c, \mu}(s)$ in Eq. (70) as

$$
W_{c, \mu}(s)=\sum_{n=1}^{\infty} \frac{e^{-s n}}{n} \int_{-|c| n}^{\infty} P_{n}(x) d x=\sum_{n=1}^{\infty} \frac{e^{-s n}}{n}\left[1-\int_{|c| n}^{\infty} P_{n}(x) d x\right] .
$$


Performing the sum, and using the definition of $W_{c, \mu}(s)$ in Eq. (70) one gets

$$
W_{c, \mu}(s)=-\ln \left(1-e^{-s}\right)-W_{|c|, \mu}(s) .
$$

For $\mu>1, W_{|c|, \mu}(0)$ is a constant as was demonstrated in the previous two subsections. Hence, one gets from Eq. (98), the leading singular behavior for small $s$

$$
W_{c, \mu}(s) \underset{s \rightarrow 0}{\longrightarrow}-\ln (s)-W_{|c|, \mu}(0)
$$

which yields, via Eq. (70)

$$
\tilde{q}(s) \underset{s \rightarrow 0}{\longrightarrow} \frac{\exp \left[-W_{|c|, \mu}(0)\right]}{s} .
$$

Thus, in this regime, the leading singularity of $\tilde{q}(s)$ occurs at $s=s^{*}=0$. Setting $s^{*}=0$ and the result (100) in the Bromwich integral in Eq. (171) gives

$$
Q(n) \underset{n \rightarrow \infty}{\longrightarrow} \alpha_{\mu}(c)=\exp \left[-W_{|c|, \mu}(0)\right]=\exp \left[-\sum_{n=1}^{\infty} \frac{1}{n} \int_{|c| n}^{\infty} P_{n}(x) d x\right] .
$$

The fact that the persistence $Q(n)$ approaches to a constant for large $n$ in this regime can be understood physically because for $c<0$ and $\mu>1$, a finite fraction of trajectories escape to $-\infty$ as $n \rightarrow \infty$.

\section{Asymptotic Record Number distribution $P(R, n)$ for large $n$}

In this section, we analyse the asymptotic large $n$ properties of the mean record number $\left\langle R_{n}\right\rangle$ and its full distribution $P(R, n)$ for arbitrary $c$ by analysing the set of equations (6), (60), (63) and (65) with arbitrary jump distribution $f(\eta)$. Consider first the mean record number. As in Section IV, we invert Eq. (65) by using the Cauchy inversion formula, deform the contour (as in Fig. (4), keep only the vertical part of the contour $C_{1}$ for large $n$ and finally make the substitution $z=e^{-s}$ to obtain the following Bromwich formula

$$
\left\langle R_{n}\right\rangle \approx \int_{s^{*}-i \infty}^{s^{*}+i \infty} \frac{d s}{2 \pi i} e^{s n} \frac{1}{\left(1-e^{-s}\right)^{2} \tilde{q}(s)},
$$

where $\tilde{q}(s)$ is given in Eqs. (69) and (70) and its small $s$ properties have already been analysed in section IV in different regimes in the $(c, 0<\mu \leq 2)$ strip. As in section IV, $s^{*}$ denotes the singularity of $\tilde{q}(s)$ on the real line in the complex plane that is closest to the origin at $s=0$.

Similarly, the record number distribution is obtained by inverting Eq. (601) in the same way

$$
P(R, n) \approx \int_{s^{*}-i \infty}^{s^{*}+i \infty} \frac{d s}{2 \pi i} e^{s n} \tilde{q}(s)\left[1-\left(1-e^{-s}\right) \tilde{q}(s)\right]^{R-1} .
$$

In this section, we use the already derived results for $\tilde{q}(s)$ in Section IV and analyse the asymptotic behavior of $\left\langle R_{n}\right\rangle$ and $P(R, n)$ respectively in Eqs. (102) and (103) in different regimes of the $(c, 0<\mu \leq 2)$ strip and on the critical line $\mu=1$. 

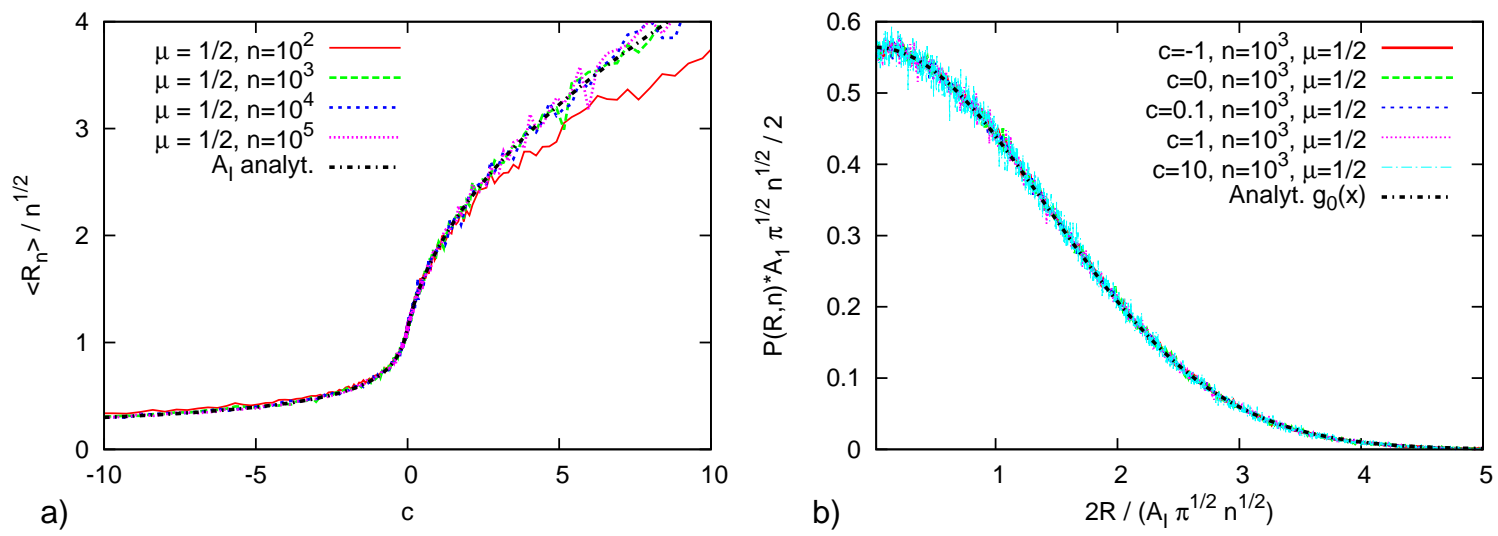

Figure 5. a): Rescaled mean record number $\left\langle R_{n}\right\rangle / \sqrt{n}$ for a Lévy-stable distribution with Lévy index $\mu=1 / 2$ and different series length $n=10^{2}, 10^{3}, 10^{4}$ and $10^{5}$. For each $n$ the average was performed over $10^{3}$ samples. For $n \gg 1$ the results collapse and agree with the predicted analytical behavior for $A_{\mathrm{I}}(c)$ in Eq. (104). b): Rescaled distribution $A_{\mathrm{I}} \sqrt{\pi n} P(R, n) / 2$ as a function of $2 R / A_{\mathrm{I}} \sqrt{\pi n}$ of the record number $R_{n}$ after $n$ steps for a random walk with a Lévy-stable jump distribution of Lévy index $\mu=1 / 2, n=10^{3}$ and different values of the drift $c=-1,0,0.1,1$ and 10 . We also plotted the asymptotic analytical result $g_{0}(x)$ given in Eq. (25). All curves collapse nicely. In regime I, the record number has a half-gaussian distribution.

\subsection{Regime I: $0<\mu<1$ and c arbitrary}

Let us first consider the asymptotic behavior of the mean number of records $\left\langle R_{n}\right\rangle$ for large $n$ in this regime. Consider the Bromwich integral in Eq. (102). For large $n$, this integral can be shown to be dominated by the small $s$ region of the integrand. Taking $s \rightarrow 0$ limit in the integrand, substituting the result (76) on the rhs of Eq. (102), and performing the Bromwich integral we get the leading asymptotic behavior for large $n$

$$
\left\langle R_{n}\right\rangle \approx A_{\mathrm{I}} \sqrt{n}, \quad \text { where } A_{\mathrm{I}}=\frac{2}{\sqrt{\pi}} e^{S_{0}}=\frac{2}{\sqrt{\pi}} \exp \left[\sum_{n=1}^{\infty} \frac{1}{n} \int_{0}^{c n} P_{n}(x) d x\right] .
$$

Comparing this to the amplitude of persistence in Eq. (78) we see that the two prefactors are related simply via $B_{\mathrm{I}}=2 /\left(\pi A_{\mathrm{I}}\right)$. The prefactor $A_{\mathrm{I}}$ can further be expressed explicitly in terms of the Fourier transform of the jump distribution $\hat{f}(k)$ as in Eq. (24). This is shown in Appendix A where we also compute the asymptotic behavior of $A_{\mathrm{I}}$ for large $|c|$ [see Eq. (A.9)]. In Fig. (5 a) we compare this result for $\left\langle R_{n}\right\rangle$ to numerical simulations. The numerical results for $n \gg 1,\left\langle R_{n}\right\rangle / \sqrt{n}$ agree nicely with our analytical values for $A_{\mathrm{I}}(c)$.

Next we turn to $P(R, n)$ in the limit of large $n$. To extract the scaling behavior of $P(R, n)$ from Eq. (103), we substitute on the rhs the small $s$ behavior of $\tilde{q}(s)$ from Eq. (76) and use the notation $e^{-S_{0}}=(2 / \sqrt{\pi}) A_{\mathrm{I}}$. The appropriate scaling limit is clearly $R \rightarrow \infty, s \rightarrow 0$ but keeping the product $\sqrt{s} R$ fixed. Taking this limit in Eq. (103) gives,

$$
P(R, n) \approx \int_{-i \infty}^{+i \infty} \frac{d s}{2 \pi i} e^{s n} \frac{2}{A_{\mathrm{I}} \sqrt{\pi s}} \exp \left[-\frac{2}{A_{\mathrm{I}} \sqrt{\pi}} \sqrt{s} R\right] .
$$




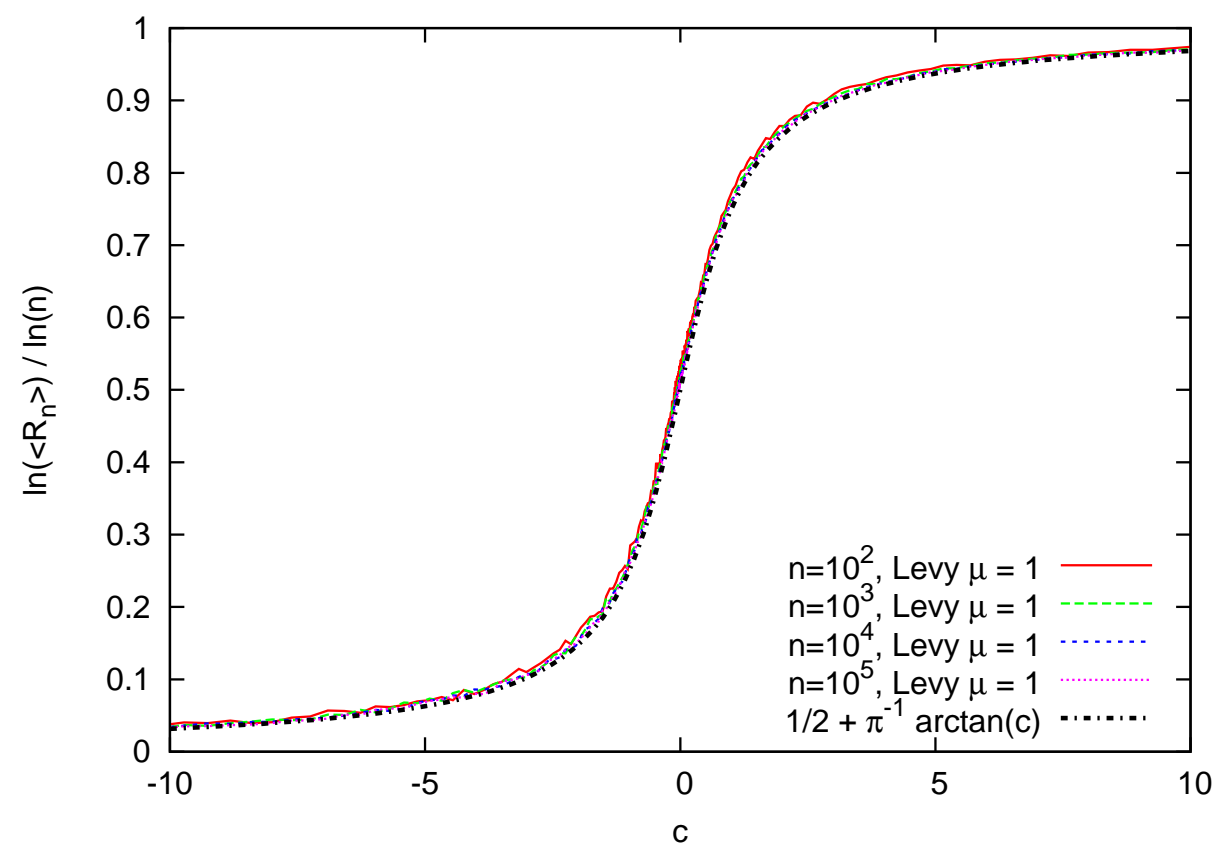

Figure 6. $\ln \left\langle R_{n}\right\rangle / \ln n$ as a function of the drift $c$ for the Cauchy distribution with Lévy index $\mu=1$ and for different values of $n=10^{2}, 10^{3}, 10^{4}$ and $10^{5}$. For each $n$ and $c$, the average was performed over $10^{3}$ samples. The results from the numerical simulations collapse and agree with the predicted analytical behavior of $\ln \left\langle R_{n}\right\rangle / \ln n=\theta(c)$ and $\theta(c)=\frac{1}{2}+\frac{1}{\pi} \arctan (c)$ as in Eq. (85).

One can simply evaluate the Bromwich integral by using the identity, $L T_{s \rightarrow n}^{-1}\left[e^{-b R \sqrt{s}} / \sqrt{s}\right]=$ $e^{-b^{2} R^{2} / 4 n} / \sqrt{\pi n}$. This leads to the asymptotic result announced in Eq. (25) in the scaling limit $n \rightarrow \infty, R \rightarrow \infty$ with the ratio $R / \sqrt{n}$ fixed. In Fig. (5b) we computed numerically the rescaled distribution $A_{\mathrm{I}} \sqrt{\pi n} P(R, n) / 2$ as a function of $2 R / A_{\mathrm{I}} \sqrt{\pi n}$ and compared it with $g_{0}(x)$ Eq. (25). The figure confirms that in regime $\mathrm{I}$, the record number has a half-Gaussian distribution with a width that depends non-trivially on the drift $c$ and the Lévy-index $\mu$.

In summary, for $0<\mu<1$, the drift is not strong enough to change the $\sqrt{n}$ growth of the mean record number. The presence of drift just modifies the prefactor of the $\sqrt{n}$ growth. Similarly, the distribution of the record number in Eq. (25) in presence of a drift, when appropriately scaled, remains unchanged from the universal half-Gaussian form in the driftless case.

\subsection{Regime II: $\mu=1$ and c arbitrary}

As mentioned in the introduction, on the critical line $\mu=1$, the record statistics was investigated in detail in Ref. 26] for the special case of Cauchy jump distribution $f_{\text {Cauchy }}(\eta)=1 /\left[\pi\left(1+\eta^{2}\right)\right]$. For a general jump distribution with $\mu=1$ (not necessarily of the Cauchy form), the record statistics has a very similar mathematical structure that can be derived from the general framework developed in this paper.

Let us first consider the growth of the mean record number $\left\langle R_{n}\right\rangle$ in Eq. (102). 
Substituting the small $s$ behavior of $\tilde{q}(s)$ from Eq. (83) and performing the Bromwich integral upon setting $s^{*}=0$ we get for large $n$

$$
\left\langle R_{n}\right\rangle \approx \frac{A_{\mathrm{II}}}{\Gamma(1+\theta(c))} n^{\theta(c)} \quad \text { where } \quad A_{\mathrm{II}}=e^{\gamma_{0}} .
$$

Note that $\gamma_{0}$ is a distribution dependent constant while the exponent $\theta(c)=$ $\int_{-\infty}^{c} \mathcal{L}_{1}(y) d y=1 / 2+\frac{1}{\pi} \arctan (c)$ is universal. In Fig. (6) this exponent is plotted and compared with numerical simulations of random walks with a Cauchy jump distribution $(\mu=1)$.

Turning now to the distribution $P(R, n)$ in Eq. (103), as before, we substitute the small $s$ expansion of $\tilde{q}(s)$ from Eq. (83). It turns out that the appropriate scaling limit for $P(R, n)$ is $n \rightarrow \infty, R \rightarrow \infty$ but keeping the ratio $R / n^{\theta(c)}$ fixed. To see this, we first set $s^{*}=0$, set $R$ large but fixed, and keep the leading terms for small $s$ to get

$$
P(R, n) \approx e^{-\gamma_{0}} \int_{-i \infty}^{+i \infty} \frac{d s}{2 \pi i} e^{s n} \frac{1}{s^{1-\theta(c)}} \exp \left[-e^{-\gamma_{0}} s^{\theta(c)} R\right]
$$

Rescaling $s n \rightarrow s$ and keeping the scaled variable $R / n^{\theta(c)}$ fixed gives the asymptotic scaling distribution

$$
P(R, n) \approx \frac{1}{A_{\mathrm{II}} n^{\theta(c)}} g_{c}\left(\frac{R}{A_{\mathrm{II}} n^{\theta(c)}}\right)
$$

where the scaling function $g_{c}(u)$, which depends continuously on $c$, is given by the formal Bromwich integral

$$
g_{c}(u)=\int_{-i \infty}^{+i \infty} \frac{d s}{2 \pi i} s^{\theta(c)-1} e^{s-u s^{\theta(c)}} \quad \text { with } \quad u \geq 0
$$

where we recall that $0 \leq \theta(c) \leq 1$

One can easily extract the tail behavior of the scaling function $g_{c}(u)$ by analysing the integral in Eq. (109). For instance, when $u \rightarrow 0, g_{c}(u)$ approaches a constant

$$
g_{c}(0)=\int_{-i \infty}^{+i \infty} \frac{d s}{2 \pi i} s^{\theta(c)-1} e^{s}=\frac{1}{\pi} \Gamma[\theta(c)] \sin [\pi \theta(c)]=\frac{1}{\Gamma[1-\theta(c)]} .
$$

The integral in Eq. (110) can be performed by wrapping the contour around the branch cut on the negative real $s$ axis.

In the opposite limit, when $u \rightarrow \infty$, the integral in Eq. (109) can be performed using the standard steepest descent method. Skipping details and using the shorthand notation $\theta=\theta(c)$ we get

$g_{c}(u \rightarrow \infty) \approx\left[2 \pi(1-\theta) \theta^{(1-2 \theta) /(1-\theta)}\right]^{-1 / 2} u^{-(1-2 \theta) / 2(1-\theta)} \exp \left[-(1-\theta) \theta^{\theta /(1-\theta)} u^{1 /(1-\theta)}\right]$.

Thus the distribution has a non-Gaussian tail. The function $g_{c}(u)$ can be expressed in terms of the one-sided Lévy distribution, which was discussed for instance in Ref. [32]. In some particular cases, the Bromwich integral in Eq. (109) can be evaluated explicitly. 


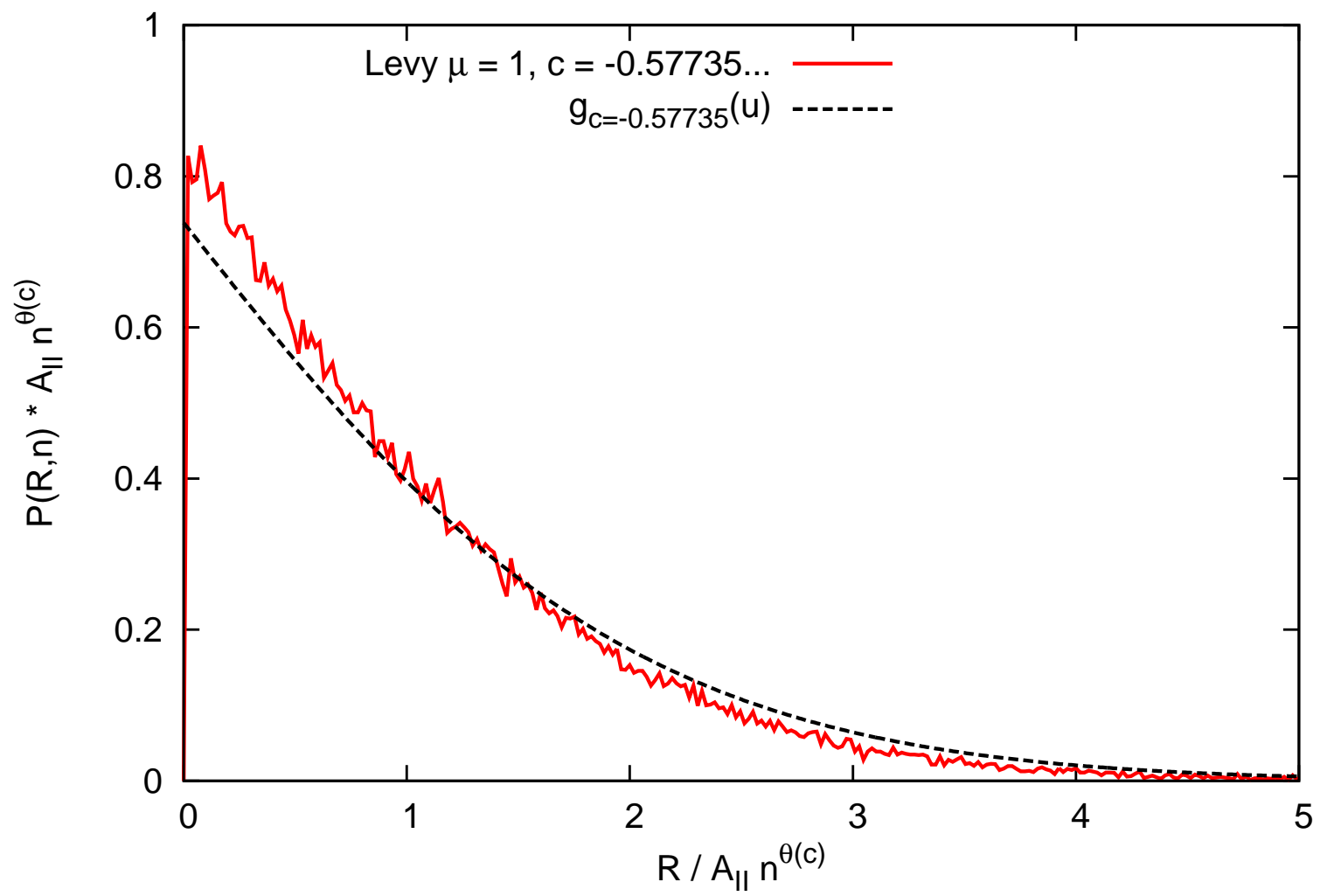

Figure 7. Rescaled plot of $A_{\mathrm{II}} n^{\theta(c)} P(R, n)$ as a function of $R / A_{\mathrm{II}} n^{\theta(c)}$ for $\mu=1$ and $c=-1 / \sqrt{3}=-0.57735$, and $\theta(c)=1 / 3$ (regime II). These data have been obtained for $n=10^{5}$ and averaged over $10^{5}$ samples. The dotted line corresponds to our exact result in Eq. (112).

For rational values of $\theta(c), g_{c}(u)$ can be expressed as a finite sum of hypergeometric functions. A very special case corresponds to $c=-1 / \sqrt{3}$ where one has $\theta=1 / 3$, such that

$$
g_{c=-1 / \sqrt{3}}(u)=3^{2 / 3} \operatorname{Ai}\left(\frac{u}{3^{1 / 3}}\right), u \geq 0 .
$$

where $\operatorname{Ai}(x)$ is the Airy function. Its asymptotic behaviors are then given by

$$
\begin{aligned}
& g_{c=-1 / \sqrt{3}}(u) \sim 1 / \Gamma(2 / 3), u \rightarrow 0 \\
& g_{c=-1 / \sqrt{3}}(u) \sim \frac{3^{3 / 4}}{2 \sqrt{\pi}} u^{-1 / 4} \exp \left(-\frac{2}{3 \sqrt{3}} u^{3 / 2}\right),
\end{aligned}
$$

which agree with the general analysis presented above (110, 111). In Fig. 7 we show a plot of the rescaled probability $A_{\mathrm{II}} n^{\theta(c)} P(R, n)$ as a function of $R / A_{\mathrm{II}} n^{\theta(c)}$ computed numerically for $c=-1 / \sqrt{3}$, which agrees reasonably well with our exact analytical result in Eq. (112). 


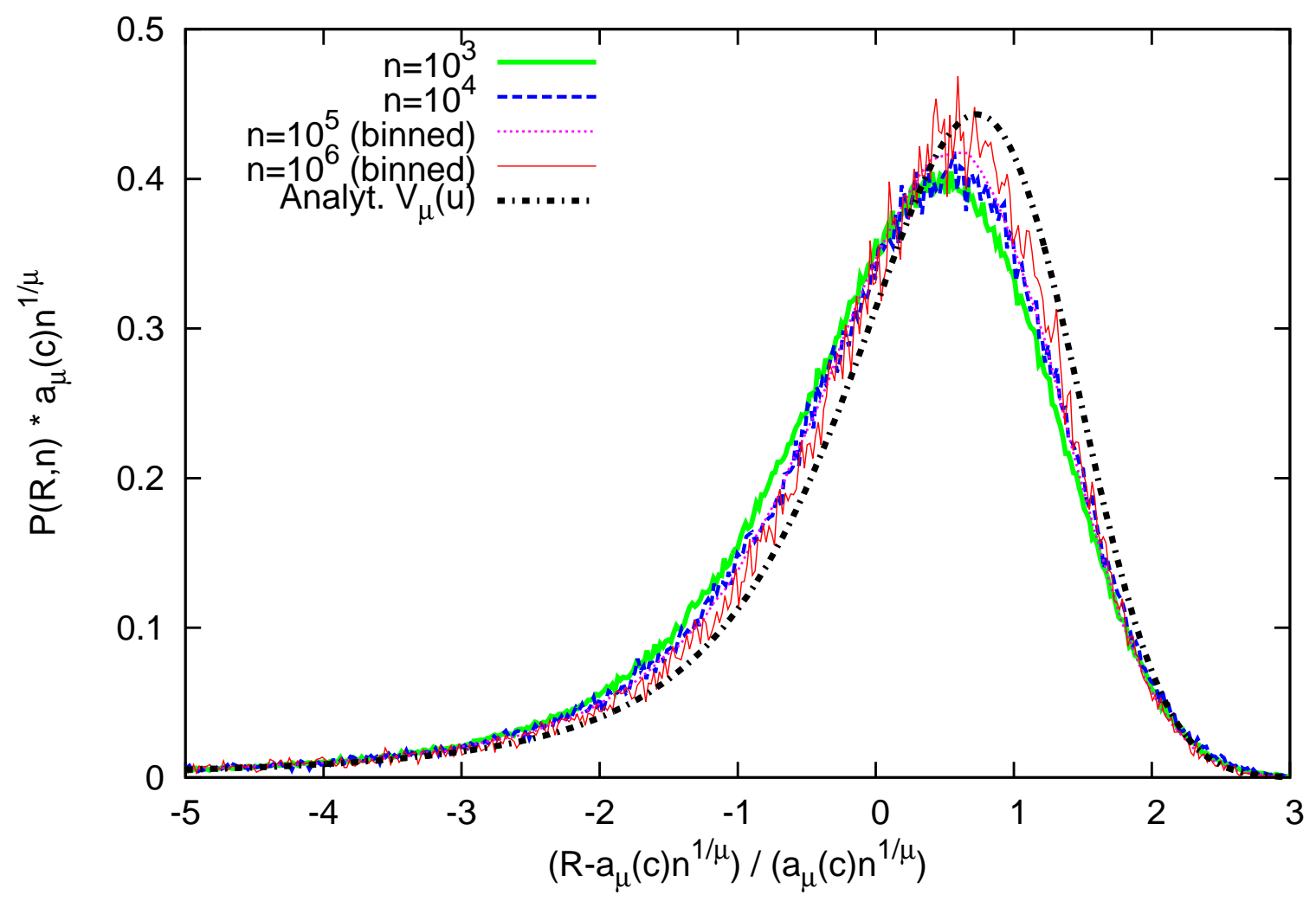

Figure 8. Rescaled distribution $a_{\mu}(c) n^{1 / \mu} P(R, n)$ of the record number $R_{n}$ after $n$ steps for a random walk with a Lévy-stable jump distribution of Lévy index $\mu=1.5$. The data are plotted as a function of the shifted and scaled variable $u=\left(R-a_{\mu}(c) n\right) /\left(a_{\mu}(c) n^{1 / \mu}\right)$. The different curves correspond to different values of $n=10^{3}, 10^{4}, 10^{5}$ and $10^{6}$ and for a drift $c=1$. They were obtained by averaging over $10^{6}$ samples. For $n=10^{5}$ and $n=10^{6}$ the numerical results were binned for technical reasons. We also plotted our analytical results for the scaling function $V_{\mu}(u)$ given by Eq. (121). While for smaller values of $n$, there is still a significant difference between the simulations and our analytical result, it converges to the behavior in Eq. (121) when $n$ increases.

\subsection{Regime III: $1<\mu<2$ and $c>0$}

We first compute the asymptotic growth of the mean number of records in this regime. Substituting the leading singular behavior of $\tilde{q}(s)$ from Eq. (88) on the rhs of Eq. (102) and performing the Bromwich integral gives

$$
\left\langle R_{n}\right\rangle \approx a_{\mu}(c) n \quad \text { where } \quad a_{\mu}(c)=\frac{1}{\tilde{q}(0)}=\exp \left[-\sum_{n=1}^{\infty} \frac{1}{n} \int_{c n}^{\infty} P_{n}(x) d x\right] .
$$

Note that we used above the expression of $\tilde{q}(0)$ in Eq. (87). We have checked numerically this linear growth and in Fig. 10 the bottom curve shows a plot of $\left\langle R_{n}\right\rangle / n$ as a function of $c$, although we have not tried to evaluate $a_{\mu}(c)$ numerically.

We next consider the distribution $P(R, n)$ in Eq. (103). We substitute the small $s$ behavior of $\tilde{q}(s)$ from Eq. (88) on the rhs of Eq. (103), set $s^{*}=0, R$ large and keep 
only leading small $s$ terms to get

$$
P(R, n) \approx \tilde{q}(0) \int_{-i \infty}^{+i \infty} \frac{d s}{2 \pi i} \exp \left[-s(\tilde{q}(0) R-n)+B_{\mu} \tilde{q}(0) R s^{\mu}\right] .
$$

Next we set

$$
R=a_{\mu}(c) n+a_{\mu}(c) n^{1 / \mu} u,
$$

where $a_{\mu}(c)=1 / \tilde{q}(0)$ and take the limit $R \rightarrow \infty, n \rightarrow \infty$ but keeping the scaled variable $u$ above fixed. We substitute Eq. (117) on the rhs of Eq. (116). Keeping only the two leading terms for large $n$ and fixed $u$ gives

$$
P(R, n) \approx \tilde{q}(0) \int_{-i \infty}^{+i \infty} \frac{d s}{2 \pi i} \exp \left[-s n^{1 / \mu} u+B_{\mu} n s^{\mu}\right]
$$

Note that for fixed $u$, both terms inside the exponential are of the same order. In fact, the scaling in Eq. (117) is chosen so as to make the two leading terms precisely of the same order for large $n$. Rescaling $s$ by $n^{1 / \mu}$, i.e., $s n^{1 / \mu} \rightarrow s$ and using $a_{\mu}(c)=1 / \tilde{q}(0)$ reduces Eq. (118) to a nicer scaling form announced in Eq. (35)

$$
P(R, n) \approx \frac{1}{a_{\mu}(c) n^{1 / \mu}} V_{\mu}(u), \quad \text { where } \quad u=\frac{R-a_{\mu}(c) n}{a_{\mu}(c) n^{1 / \mu}},
$$

and the scaling function $V_{\mu}(u)$ is formally given by the Bromwich integral

$$
V_{\mu}(u)=\int_{-i \infty}^{i \infty} \frac{d s}{2 \pi i} e^{-u s+B_{\mu} s^{\mu}}
$$

where the constant $B_{\mu}>0$ is given in Eq. (40).

Interestingly, the same scaling function $V_{\mu}(u)$ also appeared in Ref. [31] in the context of the partition function of the zero range process on a ring. The asymptotic tails of the function $V_{\mu}(u)$ were analysed in great detail in [31] (see Eqs. (78)-(83) and Fig. 5 in Ref. [31] and note that in [31], the index $\mu$ was denoted by $\gamma-1)$. We do not repeat the computations here, but just quote the results. It was found that $V_{\mu}(u)$ has highly asymmetric tails. For $u \rightarrow-\infty$, it decays as a power law, $V_{\mu}(u) \rightarrow K_{\mu}|u|^{-\mu-1}$ where the prefactor $K_{\mu}=B_{\mu} \Gamma(1+\mu) \sin [\pi(\mu+1)] / \pi$. Using our expression $B_{\mu}=-1 /(2 \cos (\mu \pi / 2))$ from Eq. (40), it is easy to show that $K_{\mu}=A_{\mu}$ where the constant $A_{\mu}$ is defined in Eq. (8). This leads to Eq. (36). In contrast, when $u \rightarrow \infty, V_{\mu}(u)$ has a faster than Gaussian tail as described precisely in Eq. (37). To plot this scaling function, a convenient real space representation can be used from Ref. [31. Replacing $\gamma-1$ by $\mu$ in Eq. (84) of Ref. [31] and using $B_{\mu}=-1 / 2 \cos (\mu \pi / 2)$, we obtain

$$
V_{\mu}(u)=\frac{1}{\pi} \int_{0}^{\infty} d y e^{-y^{\mu} / 2} \cos \left[\frac{1}{2} \tan (\mu \pi / 2) y^{\mu}+y u\right] .
$$

We compared this result for a Lévy index of $\mu=1.5$ to numerical simulations in Fig. 8 . Even though the convergence of the numerically obtained distributions is slow, it is clear 


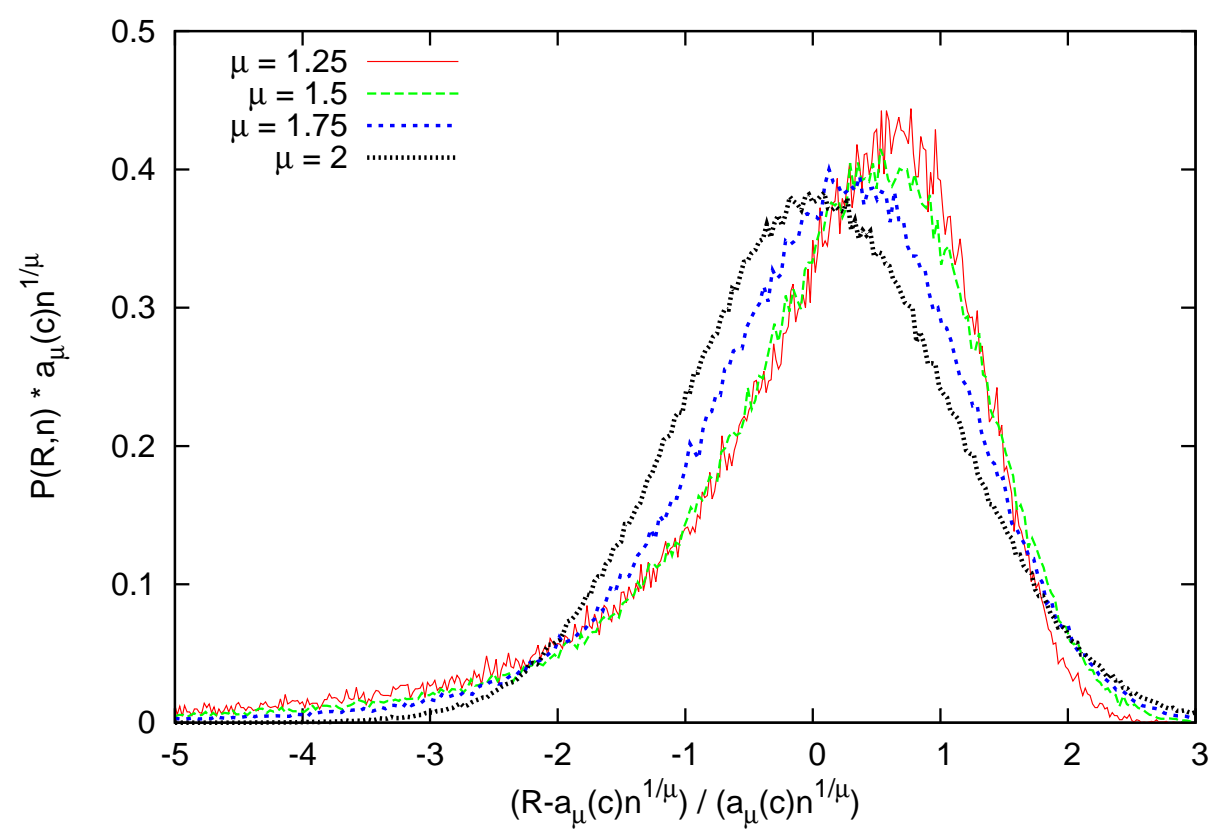

Figure 9. Rescaled distribution $a_{\mu}(c) n^{1 / \mu} P(R, n)$ of the record number $R_{n}$ after $n=10^{4}$ steps for a random walk with a Lévy-stable jump distribution with different Lévy indices $\mu=1.25,1.5,1.75$ and $\mu=2$. The data are plotted as a function of the shifted and scaled variable $u=\left(R-a_{\mu}(c) n\right) /\left(a_{\mu}(c) n^{1 / \mu}\right)$. For all these data, the value of the drift is $c=1$ and they have been obtained by averaging over $10^{6}$ samples. The figure shows that for $\mu \rightarrow 2$ this rescaled distribution approaches the Gaussian form given in Eq. (134).

that the asymptotic distribution $V_{\mu}(u)$ is approached for $n \rightarrow \infty$. In Fig. 9 we plotted numerical simulations of the rescaled record number distribution for different values of $\mu$. One finds both numerically and by taking the limit in Eq. (134) that, for $\mu \rightarrow 2$, this rescaled distribution approaches a Gaussian form (see regime IV).

To summarize, in this regime the mean record number increases linearly with increasing $n$, but the typical fluctuations around the mean are anomalously large of $\mathcal{O}\left(n^{1 / \mu}\right)$ (superdiffusive) as described in Eq. (117). In addition, the probability distribution of these typical fluctuations around the mean are described by a highly non-Gaussian form described precisely in Eq. (119).

\subsection{Regime $I V: \mu=2$ and $c>0$}

In this regime, as explained in section IV.C, $\tilde{q}(s)=\exp \left[W_{c, \mu}(s)\right]$ in Eqs. (69) and (70) is analytic at $s=0$. This can be seen by expanding the sum $W_{c, \mu}(s)$ in Eq. (70) in a Taylor series in $s$

$$
W_{c, \mu}(s)=\sum_{m=0}^{\infty} d_{m} s^{m}, \quad \text { where } \quad d_{m}=\frac{(-1)^{m}}{m !} \sum_{n=1}^{\infty} n^{m-1} \int_{c n}^{\infty} P_{n}(x) d x .
$$




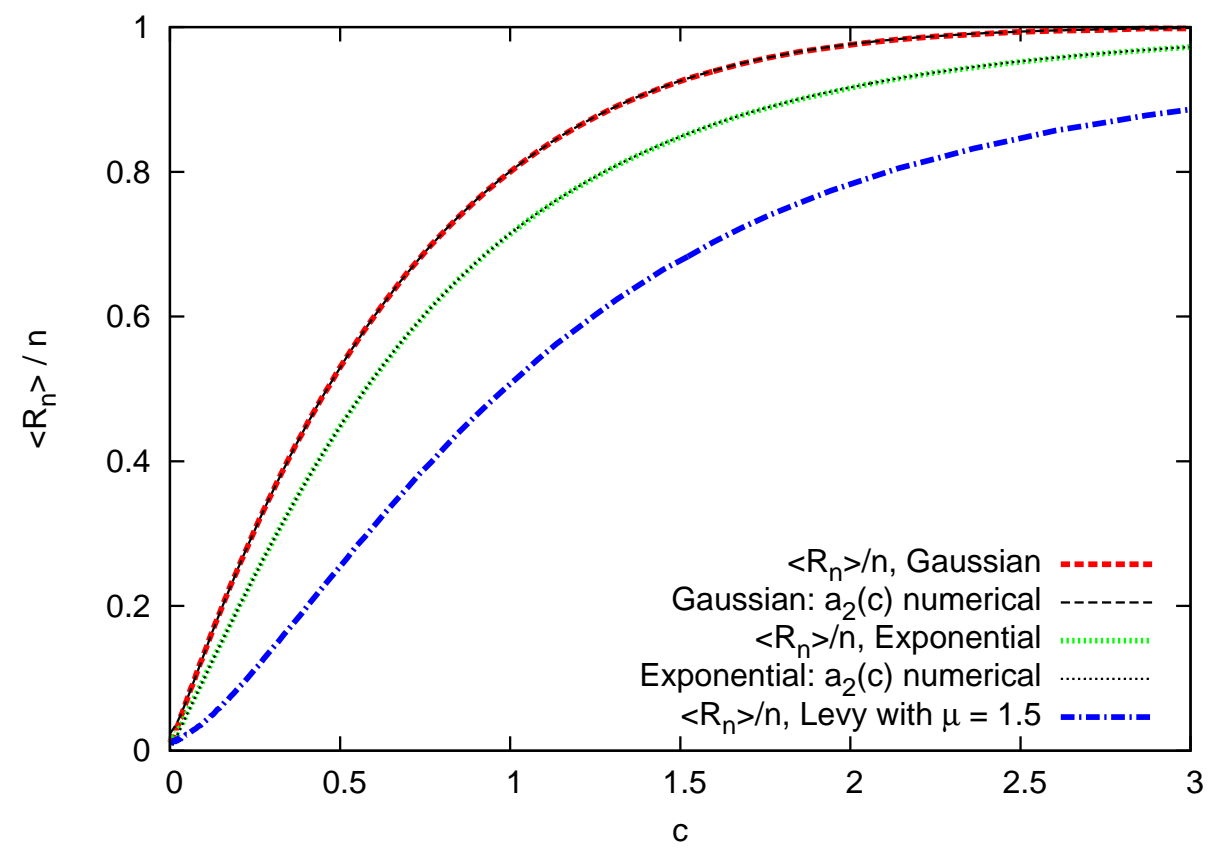

Figure 10. Numerical simulations of $\left\langle R_{n}\right\rangle / n$ for random walks with a Gaussian (with variance $\sigma=1$ ), an exponential [with parameter $b=1$, see its definition below Eq. (126)], both regime IV, and a Lévy-stable jump distribution with $\mu=1.5$, in regime III, with positive drift $c>0$. For each distribution we show data for $n=10^{4}$ which were obtained by averaging over $10^{4}$ samples. For the Gaussian and the exponential distribution we also plotted a numerical evaluation of our exact formula for $a_{2}(c)$ using Eq. (126) for the Gaussian case and Eq. (B.12) for the exponential case. Those curves agree perfectly with the numerical simulations.

The coefficient $d_{m}$, for each $m$, is finite as the sum over $n$ is convergent since the integral $\int_{c n}^{\infty} P_{n}(x) d x$ decreases with $n$ faster than exponentially for large $n$ (see section IV.C), as long as $\mu=2$ and $c>0$. Consequently, for small $s, \tilde{q}(s)$ also has a Taylor series expansion

$$
\tilde{q}(s)=\tilde{q}(0)+\tilde{q}^{\prime}(0) s+\frac{1}{2} \tilde{q}^{\prime \prime}(0) s^{2}+\ldots
$$

Let us start with the asymptotic behavior of the mean record number $\left\langle R_{n}\right\rangle$ in Eq. (102). Once again, the dominant contribution to the integral in Eq. (102) for large $n$ comes from the small $s$ region. Taking the $s \rightarrow 0$ limit in the integrand and using the small $s$ expansion in Eq. (123), keeping only the leading terms and performing the Bromwich integral term by term one gets for large $n$

$$
\left\langle R_{n}\right\rangle \approx \int_{s^{*}-i \infty}^{s^{*}+i \infty} \frac{d s}{2 \pi i} e^{s n} \frac{1}{\tilde{q}(0) s^{2}}\left[1+\left(1-\frac{\tilde{q}^{\prime}(0)}{\tilde{q}(0)}\right) s+O\left(s^{2}\right)\right] \approx a_{2}(c) n+\kappa_{2}(c)+\mathcal{O}(1 / n)
$$

where

$$
a_{2}(c)=\frac{1}{\tilde{q}(0)}=\exp \left[-\sum_{n=1}^{\infty} \frac{1}{n} \int_{c n}^{\infty} P_{n}(x) d x\right]
$$

and $\kappa_{2}(c)=\left[1-\tilde{q}^{\prime}(0) / \tilde{q}(0)\right] / \tilde{q}(0)$. 
For example, for a Gaussian jump distribution $f(\eta)=\left(2 \pi \sigma^{2}\right)^{-1 / 2} e^{-\eta^{2} / 2 \sigma^{2}}$, we have $P_{n}(x)=\left(2 \pi n \sigma^{2}\right)^{-1 / 2} e^{-x^{2} / 2 \sigma^{2} n}$ and hence $a_{2}(c)$ in Eq. (125) is given by the explicit formula

$$
a_{2}(c)=\exp \left[-\sum_{n=1}^{\infty} \frac{1}{2 n} \operatorname{erfc}\left(\frac{c \sqrt{n}}{\sigma \sqrt{2}}\right)\right] \text {. }
$$

For instance, for $c=1$ and $\sigma=1$, one gets $a_{2}(c=1)=0.800543 \ldots$ Another example is the exponential jump distribution $f(\eta)=(2 b)^{-1} \exp (-|x| / b)$. In this case, one can also compute (see the Appendix B) the constant $a_{2}(c)=\lambda$ where $\lambda$ is given by the solution of the transcendental equation $\exp (-\lambda c / b)=1-\lambda^{2}$. For example, for $c=1$, $b=1$, one gets $\lambda=0.714556 \ldots$. For these two examples, we have confirmed the leading asymptotic result for the mean record number in Eq. (124) with the exactly computed prefactors $a_{2}(c)$ (as discussed above) in our numerical simulations (see Fig. 10).

In a similar way, one can also analyse Eq. (66) for the large $n$ behavior of the second moment $\left\langle R_{n}^{2}\right\rangle$. Skipping details, we get the following leading large $n$ behavior

$$
\left\langle R_{n}^{2}\right\rangle \approx a_{2}^{2}(c) n^{2}+\rho_{2}(c) n+\mathcal{O}(1), \quad \text { where } \quad \rho_{2}(c)=\frac{1}{\tilde{q}^{2}(0)}\left[3-\tilde{q}(0)-4 \frac{\tilde{q}^{\prime}(0)}{\tilde{q}(0)}\right]
$$

Consequently, the variance of the record number grows for large $n$ as

$$
\left\langle R_{n}^{2}\right\rangle-\left\langle R_{n}\right\rangle^{2} \approx b_{2}(c) n \quad \text { where } \quad b_{2}(c)=\frac{1}{\tilde{q}^{2}(0)}\left[1-\tilde{q}(0)-2 \frac{\tilde{q}^{\prime}(0)}{\tilde{q}(0)}\right] .
$$

Thus, in this regime, the mean record number grows linearly with $n$ for large $n$ while the size of typical fluctuations around this mean grows as $\sim \sqrt{n}$.

How are these typical fluctuations around the mean distributed? To answer this, we need to analyse $P(R, n)$ in Eq. (103) in the scaling limit where both $n$ and $R$ are large, but the ratio $\left(R-a_{2}(c) n\right) / \sqrt{n}$ is fixed. To proceed, we set $s^{*}=0$ and substitute the small $s$ expansion of $\tilde{q}(s)$ in Eq. (123) on the rhs of Eq. (103), take $R$ large but fixed to get

$$
P(R, n) \approx \tilde{q}(0) \int_{-i \infty}^{+i \infty} \frac{d s}{2 \pi i} \exp \left[-s(\tilde{q}(0) R-n)+(1 / 2) b_{2}(c) \tilde{q}^{3}(0) R s^{2}\right]
$$

where $b_{2}(c)$ is given in Eq. (128). Next we set

$$
R=a_{2}(c) n+\sqrt{b_{2}(c)} \sqrt{n} u
$$

where $a_{2}(c)=1 / \tilde{q}(0)$ is given in Eq. (125) and take the scaling limit where $R \rightarrow \infty$, $n \rightarrow \infty$ but keeping the scaled variable $u$ above fixed. Substituting $R$ from Eq. (130) into Eq. (129) and keeping only the two leading terms for large $n$ gives

$$
P(R, n) \approx \tilde{q}(0) \int_{-i \infty}^{+i \infty} \frac{d s}{2 \pi i} \exp \left[-\sqrt{b_{2}(c)} \tilde{q}(0) \sqrt{n} s u+(1 / 2) b_{2}(c) \tilde{q}^{2}(0) n s^{2}\right]
$$

Note that for fixed $u$, both terms inside the exponential are of the same order. Indeed, as in the section VB, the scaling in Eq. (130) is chosen so as to make the two leading 


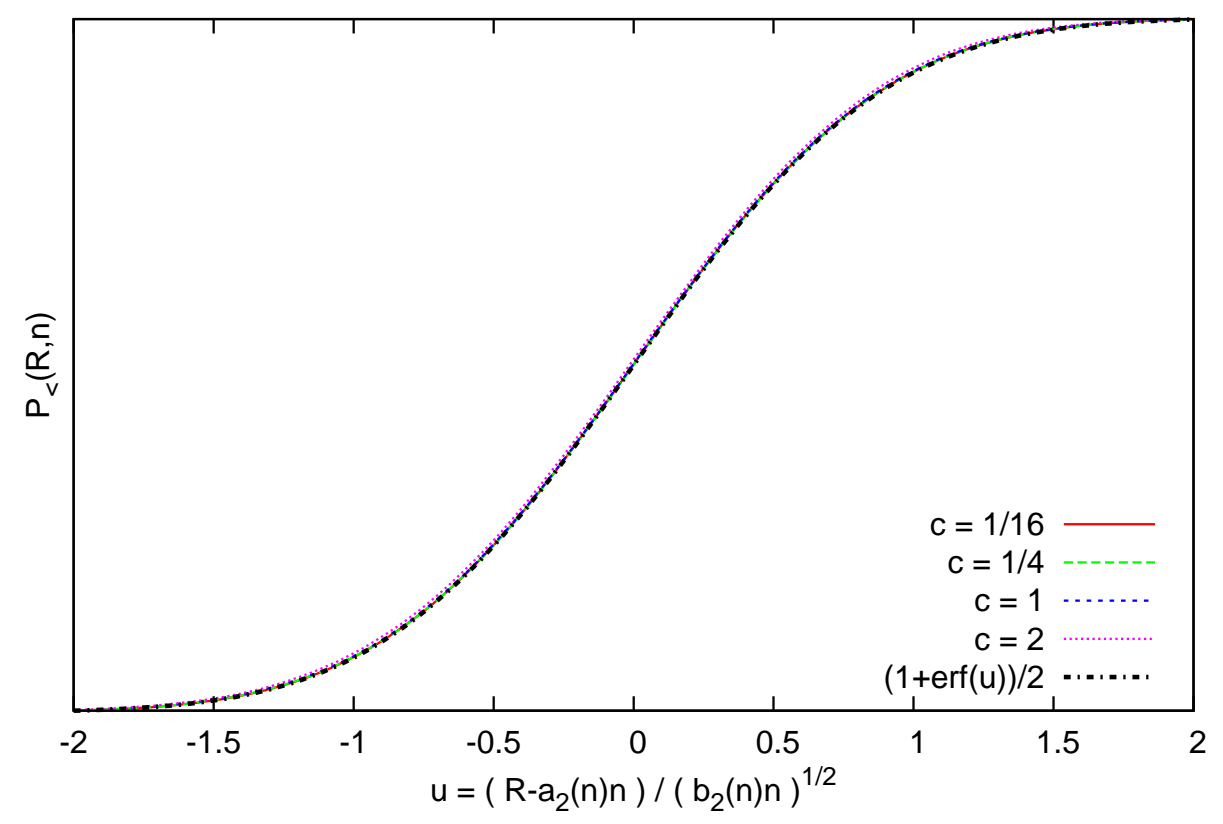

Figure 11. Plot of the cumulative distribution of record numbers $P_{<}(R, n)=$ Proba. $\left[R_{n} \leq R\right]$ as a function of the shifted and scaled variable $u=$ $\left(R-a_{2}(c) n\right) /\left(\sqrt{\left.b_{2}(c) n\right)}\right.$ for a random walk with Gaussian jump distribution (with $\sigma=1$ ) of $n=10^{4}$ steps. The different curves correspond to different values of positive drift $c=1 / 16,1 / 4,1$ and 2 . For each $c$ the data were obtained by averaging over $10^{6}$ samples. We compared the numerical results to the cumulative distribution of $V_{2}(\mu)$, which we obtained analytically (Eq. (134)). All curves collapse nicely, confirming that the asymptotic record number of a biased Gaussian random walk with a positive drift has the Gaussian distribution given by Eq. (132).

terms precisely of the same order for large $n$. Rescaling $\sqrt{b_{2}(c)} \tilde{q}(0) \sqrt{n} s \rightarrow s$ simplifies to

$$
P(R, n) \approx \frac{1}{\sqrt{b_{2}(c) n}} V_{2}(u) \text { where } u=\frac{R-a_{2}(c) n}{\sqrt{b_{2}(c) n}},
$$

and the scaling function $V_{2}(u)$ is given by the Bromwich integral

$$
V_{2}(u)=\int_{-i \infty}^{i \infty} \frac{d s}{2 \pi i} e^{-u s+s^{2} / 2},
$$

which can be exactly computed (since it is a Gaussian integral) to give

$$
V_{2}(u)=\frac{1}{\sqrt{2 \pi}} \exp \left[-u^{2} / 2\right] .
$$

This then proves that $P(R, n)$ is asymptotically Gaussian as announced in Eq. (45). Fig. 11]confirms this result numerically. We plotted the cumulative distribution of record numbers $P_{<}(R, n)=$ Proba. $\left[R_{n} \leq R\right]$ as a function of the shifted and scaled variable $u=\left(R-a_{2}(c) n\right) /\left(\sqrt{\left.b_{2}(c) n\right)}\right.$ after $n=10^{4}$ steps for different values of positive drift $c$ and compared them to a Gaussian cdf (cumulative distribution function). All numerical results collapsed perfectly on the analytical curve. 


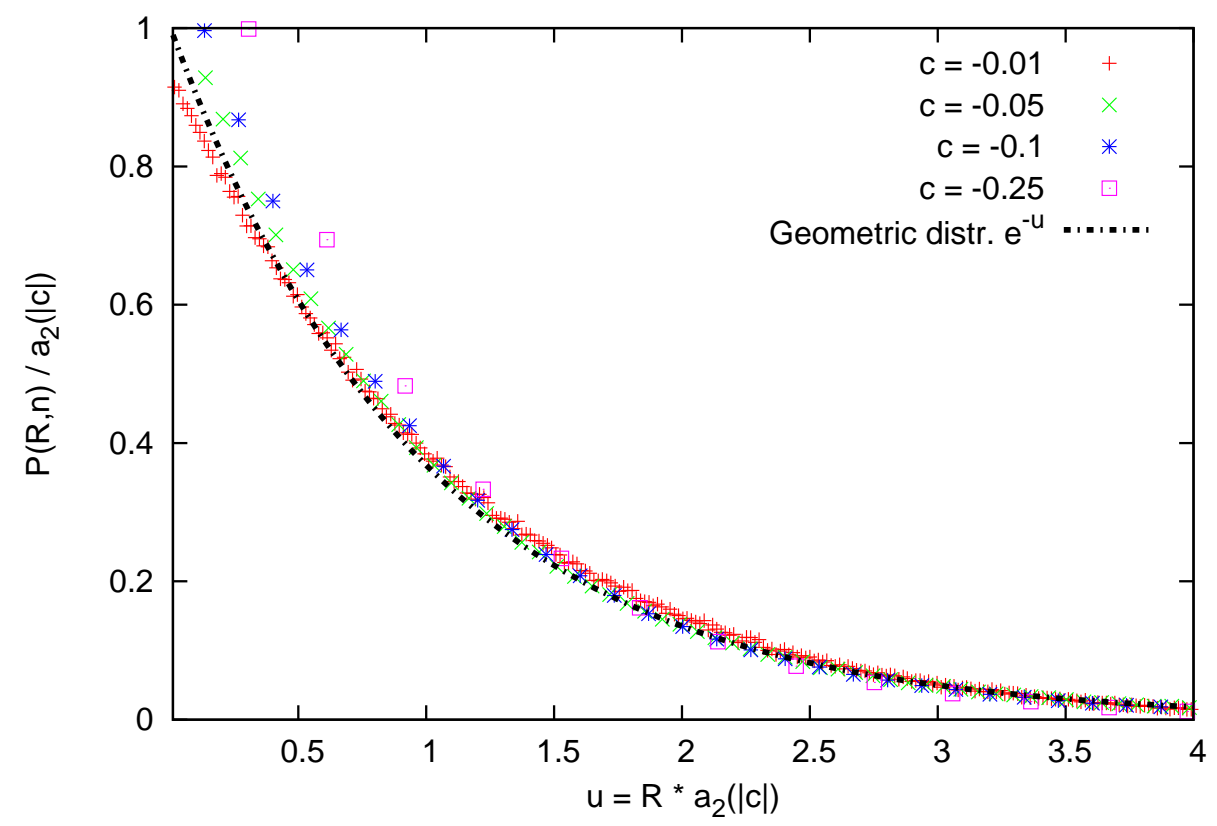

Figure 12. Rescaled distribution $a_{2}(|c|) P(R, n)$ of the record number $R_{n}$ after $n=10^{4}$ steps for a random walk with a Gaussian jump distribution, of variance $\sigma=1$, with different negative values of the drift $c=-0.01, c=-0.05,-0.1$ and -0.25 . The data are plotted as a function of the rescaled variable $u=R a_{2}(|c|)$. For each value of $c$ the data were obtained by averaging over $10^{4}$ samples. We compared the numerical results with a simple geometric distribution. The good agreement confirms our analytical findings given by Eq. (136).

\subsection{Regime $V: 1<\mu \leq 2$ and $c<0$}

In this regime, we set $s^{*}=0$ in Eq. (103) and substitute on its rhs the small $s$ expansion of $\tilde{q}(s)$ from Eq. (100). Keeping only leading order behavior for small $s$ gives, for large $n$,

$$
P(R, n) \approx \alpha_{\mu}(c)\left[1-\alpha_{\mu}(c)\right]^{R-1} \int_{s^{*}-i \infty}^{s^{*}+i \infty} \frac{d s}{2 \pi i} e^{s n} \frac{1}{s},
$$

where the constant $\alpha_{\mu}(c)=\exp \left[-W_{|c|, \mu}(0)\right]=\exp \left[-\sum_{n=1}^{\infty} \frac{1}{n} \int_{|c| n}^{\infty} P_{n}(x) d x\right]$ as given in Eq. (101).

Using the fact that $L T_{s \rightarrow n}^{-1}[1 / s]=1$ gives the large $n$ (but $R$ fixed) behavior of $P(R, n)$

$$
P(R, n) \underset{n \rightarrow \infty}{\longrightarrow} \alpha_{\mu}(c)\left[1-\alpha_{\mu}(c)\right]^{R-1} .
$$

Thus, the distribution becomes independent of $n$ for large $n$ and has a simple geometric form with mean $\left\langle R_{n}\right\rangle \rightarrow 1 / \alpha_{\mu}(c)$. Comparing the expression of $\alpha_{\mu}(c)$ as given in Eq. (101) and those of $a_{\mu}(c)$ in Eq. (115) and $a_{2}(c)$ in Eq. (125) for $c>0$, one immediately finds that $\alpha_{\mu}(c)=a_{\mu}(|c|)$ for $1<\mu<2$ while $\alpha_{2}(c)=a_{2}(|c|)$, the results mentioned respectively in Eqs. (501) and (51).

In Fig. 12 we compared Eq. (136) to numerical simulations of negatively biased 
Gaussian random walks with different values of $c$. For large $n$ the rescaled distribution of $u=R a_{2}(|c|)$ approaches the geometric (exponential) distribution $e^{-u}$.

\section{Extreme statistics of the age of a record}

From the previous study of the mean number of records $\left\langle R_{n}\right\rangle$, one deduces that the typical age (see Fig. 2) ) of a record is given by $l_{\text {typ }} \sim n /\left\langle R_{n}\right\rangle$. However, following Ref. [22] for the unbiased case, it turns out that the extreme ages of records do not share the typical behavior. In this section, we probe such atypical extremal statistics by considering the longest and shortest lasting records characterized by their respective ages (durations) $l_{\max , n}$ and $l_{\min , n}$. We focus on their mean values $\left\langle l_{\max , n}\right\rangle,\left\langle l_{\min , n}\right\rangle$ and find rather different asymptotic behaviors in the five regimes in the $(c, 0<\mu \leq 2)$ strip mentioned before (Fig. 11).

\subsection{Age of the longest lasting record $l_{\max , n}$}

We first consider the longest lasting record whose age $l_{\max , n}$ is given by (see Fig. 2)

$$
l_{\max }=\max \left(l_{1}, l_{2}, \cdots, l_{R}\right) .
$$

The cumulative distribution $\mathcal{F}_{n}(m)=$ Proba. $\left(l_{\max , n} \leq m\right)$ was studied in Ref. [22], where an explicit formula for its generating function (GF) was obtained:

$$
\sum_{n=0}^{\infty} \mathcal{F}_{n}(m) z^{n}=\frac{\sum_{l=1}^{m} Q(l) z^{l}}{1-\sum_{l=1}^{m} F(l) z^{l}}
$$

where $F(l)=Q(l-1)-Q(l)$, from which one deduces the generating function of the mean $\left\langle l_{\max , n}\right\rangle=\sum_{m=1}^{\infty}\left[1-\mathcal{F}_{n}(m)\right]$

$$
\begin{aligned}
& \sum_{n=0}^{\infty} z^{n}\left\langle l_{\max , n}\right\rangle=\sum_{m=1}^{\infty}\left[\frac{1}{1-z}-\frac{\sum_{l=1}^{m} Q(l) z^{l}}{1-\sum_{l=1}^{m} F(l) z^{l}}\right] \\
& =\frac{1}{1-z} \sum_{m=1}^{\infty} \frac{\sum_{l=m}^{\infty} F(l) z^{l}+(1-z) \sum_{l=m}^{\infty} Q(l) z^{l}}{(1-z) \tilde{Q}(z)+\sum_{l=m}^{\infty} F(l) z^{l}}
\end{aligned}
$$

where we have used that $\tilde{F}(z)=1-(1-z) \tilde{Q}(z)$ (58).

In the absence of drift, $c=0$, it was shown in Ref. [22] that $\left\langle l_{\max , n}\right\rangle$ behaves, for large $n$, linearly with $n$ with a non trivial coefficient, independently of the jump distribution $f(\eta)$

$$
\left\langle l_{\max , n}\right\rangle \sim C_{0} n, C_{0}=\int_{0}^{\infty} d y \frac{1}{1+y^{1 / 2} e^{y} \int_{0}^{y} d x x^{-1 / 2} e^{-x}}=0.626508 \ldots
$$

Interestingly, this constant $C_{0}$ appears also in the study of the longest excursion of Brownian motion [33, 34]. Note that to obtain the large $n$ behavior of $\left\langle l_{\max , n}\right\rangle$ from Eq. (139) one has to analyse the above formula (139) in the limit $z \rightarrow 1$. We will see that in this limit the above sum over $m$ is dominated by the large values of $m$, which thus depends crucially on the large $m$ behavior of the persistence probability $Q(m)$. 
Consequently $\left\langle l_{\max , n}\right\rangle$ behaves quite differently in the five regimes in the $(c, 0<\mu \leq 2)$ strip in Fig. 1 and are summarized as follows:

$$
\begin{aligned}
\left\langle l_{\max , n}\right\rangle & \sim n \quad \text { for } 0<\mu<1 \text { and } c \text { arbitrary } \quad \text { (regime } \mathrm{I}), \\
& \sim n \text { for } \mu=1 \text { and } c \text { arbitrary } \quad(\text { regime } \mathrm{II}), \\
& \sim n^{\frac{1}{\mu}} \text { for } 1<\mu<2 \text { and } c>0 \quad(\text { regime } \mathrm{III}) \\
& \sim \ln n \text { for } \quad \mu=2 \text { and } c>0 \quad(\text { regime } \mathrm{IV}) \\
& \sim n \text { for } 1<\mu \leq 2 \text { and } c<0 \quad(\text { regime } \mathrm{V}) .
\end{aligned}
$$

In the following we will discuss the behavior of $\left\langle l_{\max , n}\right\rangle$ separately for the five regimes.

6.1.1. Regime I: $0<\mu<1$, c arbitrary: In this regime, we remind that $Q(m)$ behaves, for large $m$, as

$$
Q(m) \sim \frac{B_{\mathrm{I}}}{\sqrt{m}}, F(m) \sim \frac{B_{\mathrm{I}}}{2 m^{3 / 2}},
$$

where $B_{\mathrm{I}}$ is given in Eq. (178). Setting $z=e^{-s}$ we are interested in the limit $s \rightarrow 0$ in

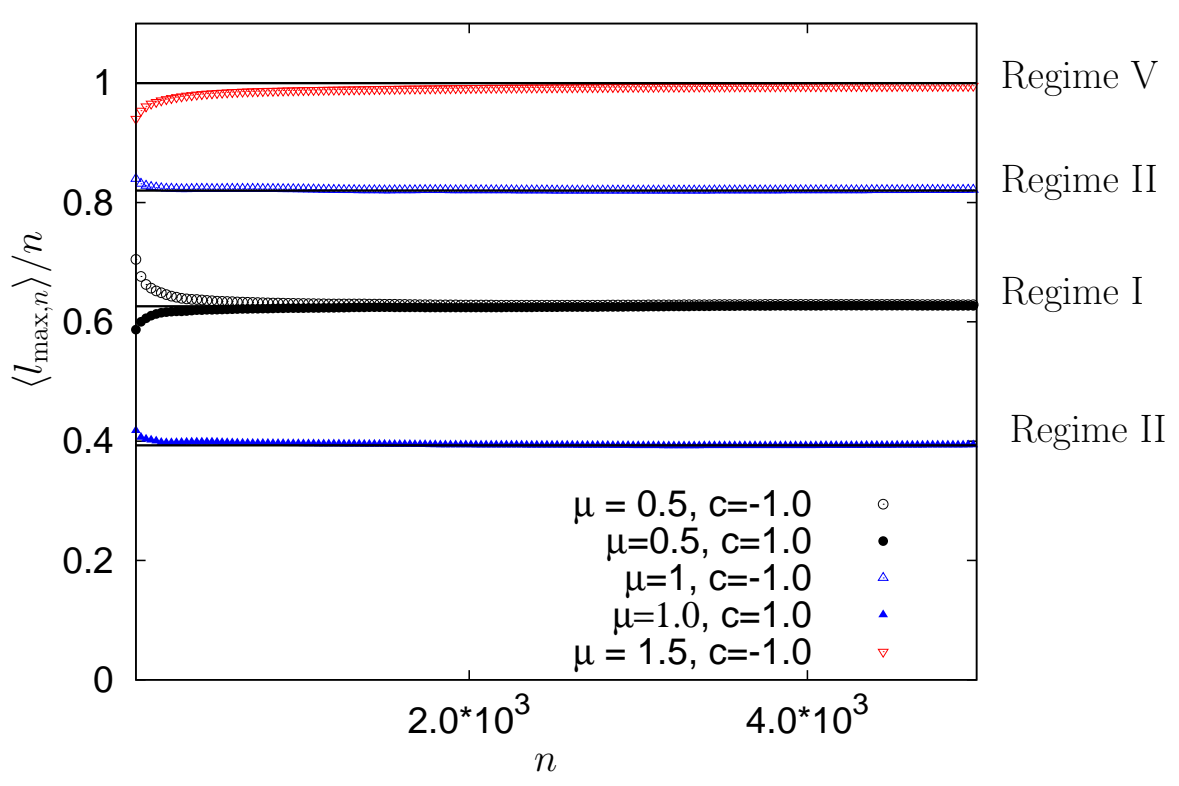

Figure 13. Plot of $\left\langle l_{\max , n}\right\rangle / n$ in the different regimes I, II and V: the points are the results of our numerical simulations. For regime II $(\mu=1)$, we present two curves, one with a negative drift $(c=-1)$ (the second curve from top) and one with a positive drift $(c=1)$ (the bottom curve). These data indicate that in all these cases $\left\langle l_{\max , n}\right\rangle \propto n$, for large $n$, with an amplitude which agree quite well with our analytical results, which are represented in solid line for each of these cases and corresponds to the formula given in Eq. (145, 147, 158). 
the formula in Eq. (139) where one can replace $F(m)$ and $Q(m)$ by their asymptotic behaviors

$$
\sum_{n=0}^{\infty}\left\langle l_{\max , n}\right\rangle e^{-s n} \sim \frac{1}{s} \sum_{m=1}^{\infty} \frac{\frac{1}{2} \sum_{l=m}^{\infty} l^{-3 / 2} e^{-s l}+s \sum_{l=m}^{\infty} l^{-1 / 2} e^{-s l}}{\sqrt{\pi} s^{1 / 2}+\frac{1}{2} \sum_{l=m}^{\infty} l^{-3 / 2} e^{-s l}},
$$

where we have used $\tilde{q}(s) \sim \sqrt{\pi} B_{\mathrm{I}} / \sqrt{s}$ when $s \rightarrow 0$ (76, 78). In the limit $s \rightarrow 0$, the discrete sums over $l$ and $m$ can be replaced by integrals and one finds that the right hand side in Eq. (144) behaves like $1 / s^{2}$ when $s \rightarrow 0$ with a prefactor which we can compute to obtain the large $n$ behavior of $\left\langle l_{\max , n}\right\rangle$ as

$$
\left\langle l_{\max , \mathrm{n}}\right\rangle \sim C_{\mathrm{I}} n, C_{\mathrm{I}}=\int_{0}^{\infty} d y \frac{y^{-1 / 2} e^{-y}}{\sqrt{\pi}+\frac{1}{2} \int_{y}^{\infty} d x x^{-3 / 2} e^{-x}}=C_{0},
$$

where $C_{0}$ is given above (141) and where the last equality is simply obtained by performing an integration by part in the integral over $x$ in the denominator. In Fig. 13, we have plotted the results of our numerical estimate of $\left\langle l_{\max , n}\right\rangle$ (obtained by averaging over $10^{4}$ different realizations of random walks) for $\mu=0.5$ and two different values of $c= \pm 1.0$. This plot shows that $\left\langle l_{\max , n}\right\rangle / n$ saturates rather quickly to the constant $C_{0}$, independently of $c$, in agreement with Eq. (145).

Thus in this regime the large $n$ behavior of $\left\langle l_{\max , n}\right\rangle$ is unaffected by the presence of the drift $c$. This result could have been anticipated as $l_{\max , n}$ can be considered as the longest excursion between two consecutive zeros of a renewal process with a persistence exponent 1/2. This quantity was studied in Ref. [34] and its average was computed, yielding the large $n$ behavior obtained in Eq. (145).

6.1.2. Regime $I I: \mu=1$ and c arbitrary: In this regime, we recall that the persistence probability $Q(m)$ behaves algebraically for large $m$ with an exponent $\theta(c)$ which depends continuously on $c$

$$
Q(m) \sim \frac{B_{\mathrm{II}}}{m^{\theta(c)}}, \quad \theta(c)=\frac{1}{2}+\frac{1}{\pi} \arctan (c),
$$

where the amplitude $B_{\mathrm{II}}$ is given in Eq. (85). Here again we can use the result obtained in Ref. [34] for the longest excursion between consecutive zeros of a renewal process with a persistence exponent $\theta(c)$ to obtain

$$
\left\langle l_{\max , n}\right\rangle \sim C_{\mathrm{II}} n, \quad C_{\mathrm{II}}=\int_{0}^{\infty} d y \frac{1}{1+y^{\theta(c)} e^{y} \int_{0}^{y} d x x^{-\theta(c)} e^{-x}},
$$

which depends continuously on $c$ and is independent of the non-universal amplitude $B_{\text {II }}$ (146). In Fig. 14 we show a comparison of $C_{\mathrm{II}}$ obtained numerically (the squares symbols) and from our exact formula (solid line), which shows a very good agreement between both.

6.1.3. Regime III: $1<\mu<2$ and $c>0$ : In this regime the persistence probability $Q(m)$ behaves for large $m$ as

$$
Q(m) \sim \frac{B_{\mathrm{III}}}{m^{\mu}},
$$




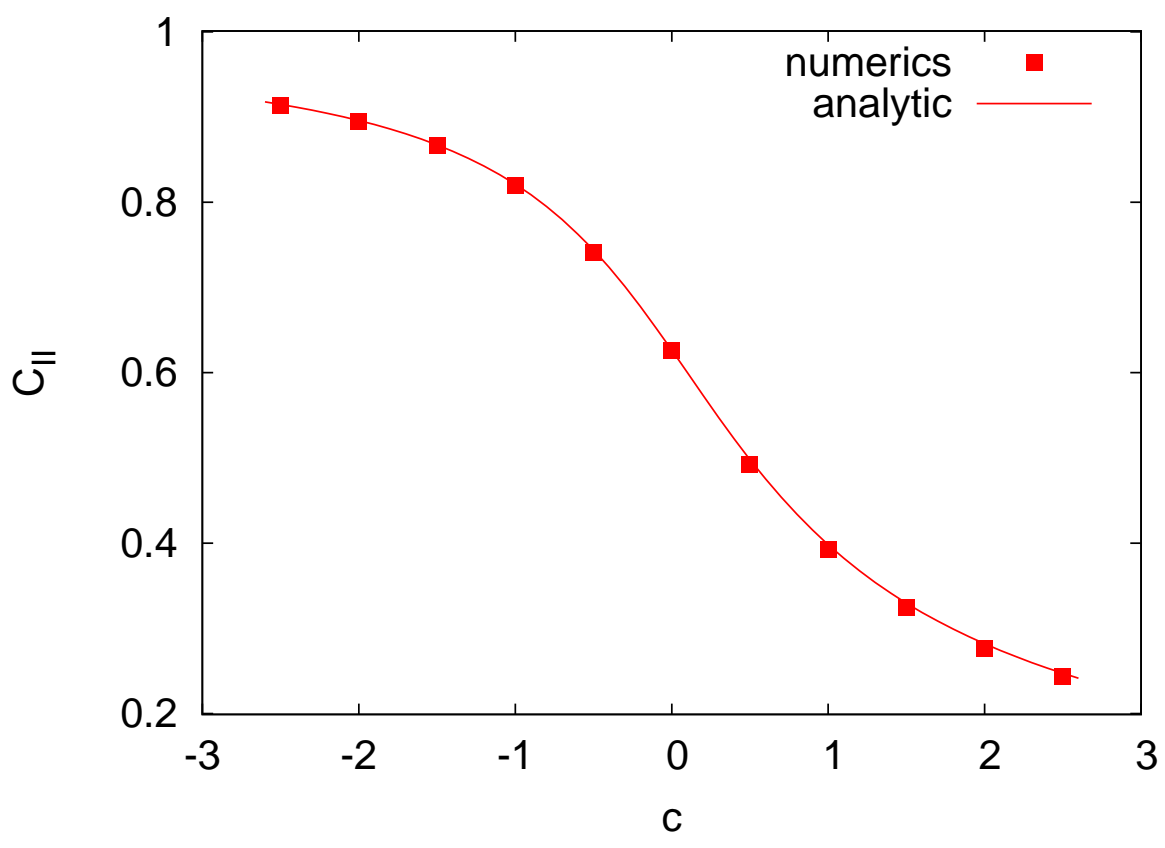

Figure 14. Plot of $C_{\mathrm{II}}$ as a function of $c$. The red squares correspond to numerical data while the solid line corresponds to our analytical result in Eq. (147) together with Eq. (146).

where the amplitude $B_{\mathrm{III}}$ is given in Eq. (90). Using again the results obtained in Ref. [34] one obtains that

$$
\left\langle l_{\max , n}\right\rangle \sim C_{\text {III }} n^{1 / \mu},
$$

where, however, the amplitude $C_{\mathrm{III}}$ was not given in Ref. [34]. A careful analysis of the above formula (139) allows to obtain the amplitude $C_{\text {III }}$ as

$$
C_{\mathrm{III}}=\frac{1}{c} \Gamma(1-1 / \mu)\left[\frac{1}{\pi} \sin \left(\frac{\mu \pi}{2}\right) \Gamma(\mu)\right]^{1 / \mu},
$$

which diverges as $C_{\mathrm{III}} \sim(\pi(\mu-1))^{-1}$ when $\mu \rightarrow 1$ and vanishes as $C_{\mathrm{III}} \sim \sqrt{\pi(2-\mu) / 2}$ when $\mu \rightarrow 2$. In Fig. 15 we show a plot of our numerical data for $\left\langle l_{\max , n}\right\rangle$ (averaged again over $10^{4}$ different realizations) for different values of $\mu=1.4,1.5,1.7,1.9$ and for a fixed value of the drift $c=5.0$. The solid lines indicate the corresponding exact asymptotic behaviors in Eq. (149, 150): the agreement between the two is quite good although the convergence to the asymptotic behavior gets slower as $\mu$ decreases to 1 .

6.1.4. Regime $I V: \mu=2$ and $c>0$ : In this case the persistence $Q(m)$ behaves quite differently as it vanishes exponentially for large $m$ as

$$
Q(n) \sim \frac{B_{\mathrm{IV}}}{n^{3 / 2}} e^{-s_{1} n} \quad \text { where } \quad s_{1}=\frac{c^{2}}{2 \sigma^{2}},
$$


$*[\mathrm{ht}]$

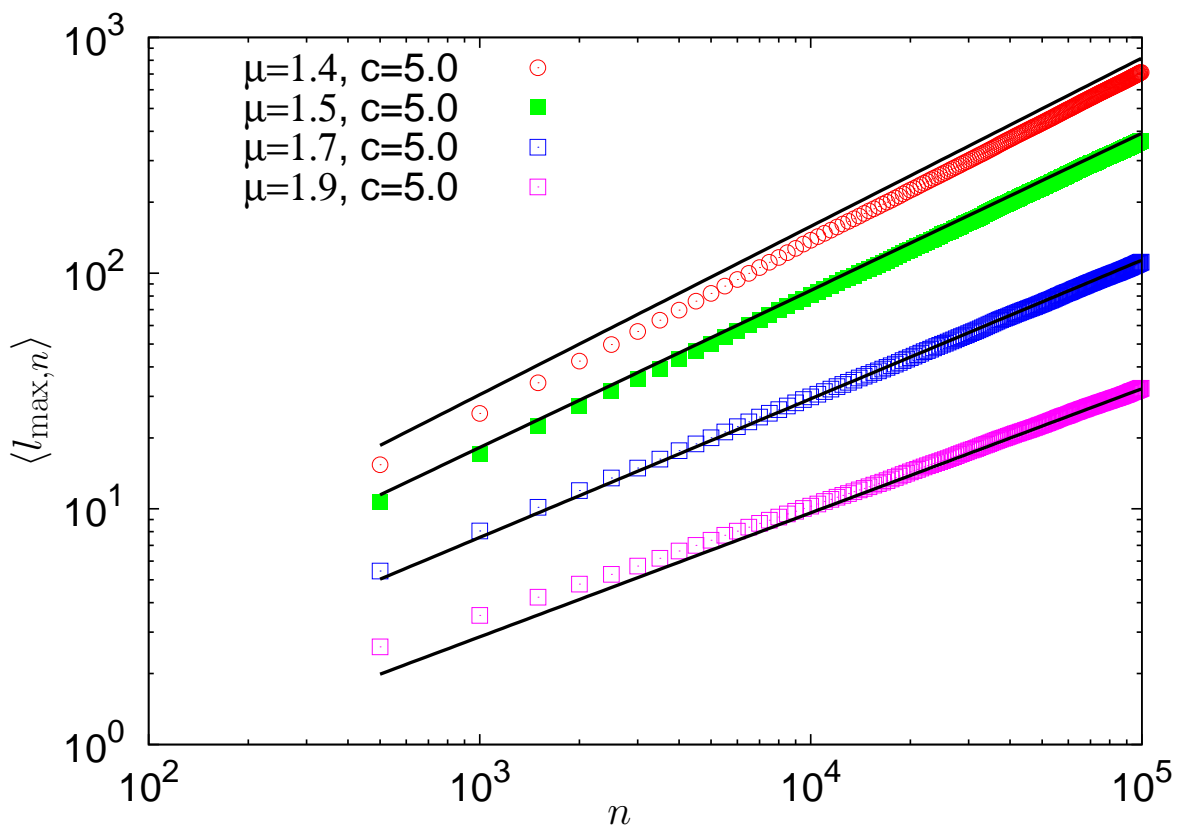

Figure 15. Plot, in a $\log -\log$ scale, of $\left\langle l_{\max , \mathrm{n}}\right\rangle$ as a function of $n$ in regime III: the different curves correspond to different values of $\mu=1.4,1.5,1.7,1.9$ with a fixed value of $c=5.0$. The solid line are the exact results given in Eqs (149, 150), without any fitting parameter.

where the amplitude $B_{\mathrm{IV}}$ is given in Eq. (96). This case was not analyzed in Ref. [34]. From Eq. (139) one has in this case

$$
\sum_{n=0}^{\infty}\left\langle l_{\max , n}\right\rangle e^{-s n} \sim \frac{1}{s} \sum_{m=1}^{\infty} \frac{\sum_{l=m}^{\infty} F(l)}{s \tilde{q}(0)+\sum_{l=m}^{\infty} F(l)}=\frac{1}{s} \sum_{m=1}^{\infty} \frac{Q(m)}{s \tilde{q}(0)+Q(m)} .
$$

Therefore in the limit when $s \rightarrow 0$ one can estimate the leading behavior of the sum over $m$ as

$$
\sum_{n=0}^{\infty}\left\langle l_{\max , n}\right\rangle e^{-s n} \sim \frac{m^{*}}{s},
$$

where $m^{*}$ is such that

$$
Q\left(m^{*}\right) \sim s \tilde{q}(0) .
$$

From the asymptotic behavior above (151) one finds that $m^{*} \sim-\frac{\sigma^{2}}{2 c^{2}} \ln s$ so that finally

$$
\left\langle l_{\max , n}\right\rangle \sim C_{\mathrm{IV}} \ln n, C_{\mathrm{IV}}=\frac{2 \sigma^{2}}{c^{2}},
$$

which is in sharp contrast with the algebraic growth obtained above in Eq. (149) for $1<\mu<2$ and $c>0$. In Fig. 16 we show a plot of $\left\langle l_{\max , n}\right\rangle$ as a function of $\ln n$ : the straight line suggests indeed a logarithmic growth, in agreement with our analytic result (155). However, a more precise comparison with this exact asymptotic result, as 


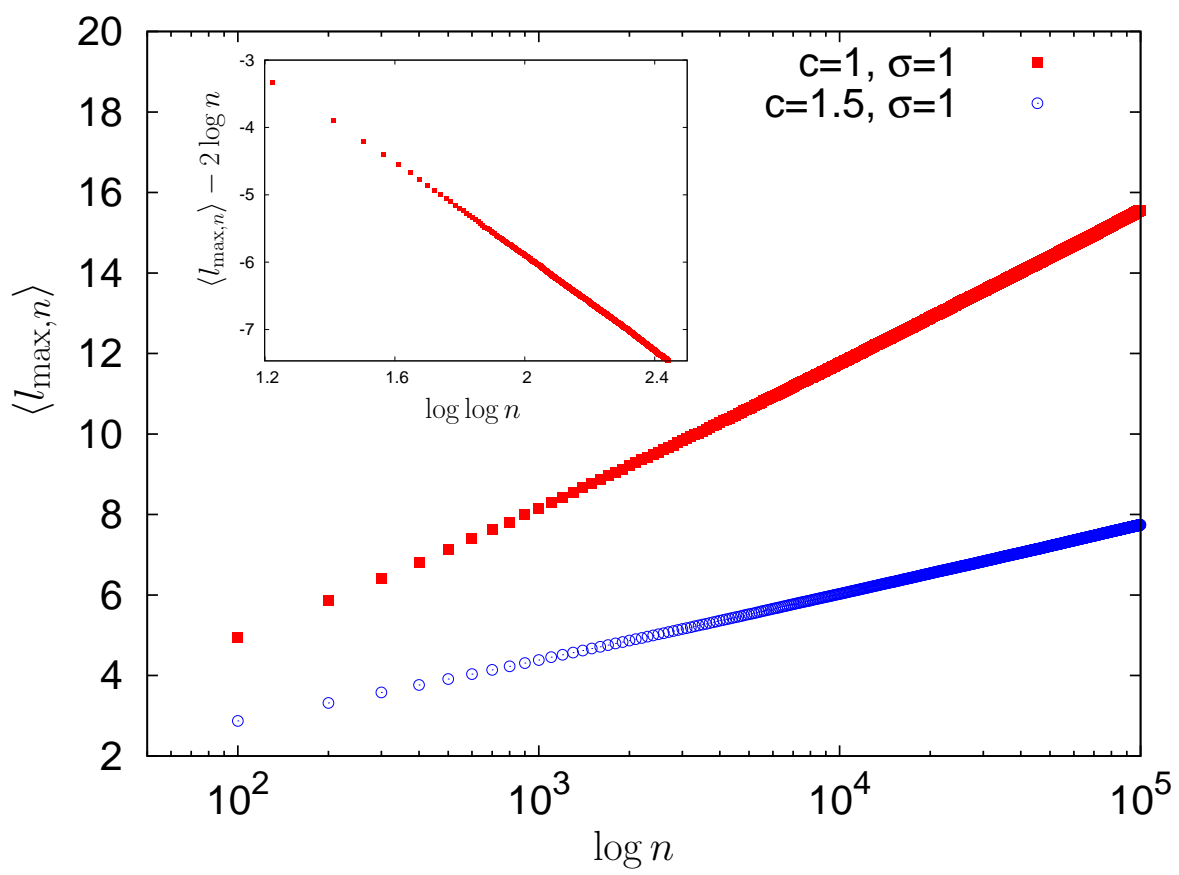

Figure 16. Plot of $\left\langle l_{\max , n}\right\rangle$ as a function of $\ln n$ in the regime IV: here $\mu=2$ and the two curves correspond to $c=1$ and $c=1.5$ ( $\sigma=1$ in both cases). The two curves suggest a logarithmic growth, as expected from Eq. (155). Inset: Plot of $\left\langle l_{\max , n}\right\rangle-2 \ln n$ where $2 \ln n$ is the exact asymptotic result from Eq. (155) and $2 \sigma^{2} / c^{2}=2$. This plot suggests rather strong corrections $\propto \ln \ln n$ to the leading logarithmic growth of $\left\langle l_{\max , n}\right\rangle$.

shown in the inset of Fig. 16, suggests that the leading corrections are proportional to $\ln \ln n$, and hence quite strong.

6.1.5. Regime $V: 1<\mu \leq 2$ and $c<0$ : In this case the persistence probability $Q(m)$ tends asymptotically to a constant (101):

$$
Q(m) \underset{m \rightarrow \infty}{\longrightarrow} \alpha_{\mu}(c)=\exp \left[-W_{|c|, \mu}(0)\right]=\exp \left[-\sum_{n=1}^{\infty} \frac{1}{n} \int_{|c| n}^{\infty} P_{n}(x) d x\right] .
$$

In addition from (86) one has that $Q(m)-\alpha_{\mu}(c) \propto n^{\mu-1}$ so that $F(m) \propto m^{-\mu}$ for large $m$. Therefore, the terms entering into the sum in Eq. (139) are given, to leading order when $1-z=e^{-s} \rightarrow 0$ and large $m$ (which are terms which give the leading contribution to this sum over $m$ )

$$
\frac{\sum_{l=m}^{\infty} F(l) z^{l}+(1-z) \sum_{l=m}^{\infty} Q(l) z^{l}}{(1-z) \tilde{Q}(z)+\sum_{l=m}^{\infty} F(l) z^{l}} \sim \frac{\alpha_{\mu}(c)}{\tilde{q}(0)} e^{-s m}=e^{-s m} .
$$

Therefore this yields

$$
\left\langle l_{\max , n}\right\rangle \sim C_{\mathrm{V}} n, C_{\mathrm{V}}=1 .
$$


This result, which is corroborated by our numerical simulations (see Fig. 13), can be physically understood as in this regime where $c<0$ and $\mu>1$ the number of records is finite and these records typically occur during the first steps of the random walks, where the walker might stay positive for a short while before it escapes to negative values when $n \rightarrow \infty$, and no record happens any more.

\subsection{Age of shortest lasting record $l_{\min , n}$}

We now consider the shortest lasting record whose age $l_{\min , n}$ is given by (see Fig. 2)

$$
l_{\min , n}=\min \left(l_{1}, l_{2}, \cdots, l_{R}\right) .
$$

Note that, given that the final incomplete interval $l_{R}$ is taken into consideration above, $l_{\min , n}$ can be zero: this happens when a record has been broken at the last step, such that $l_{R}=0$.

The cumulative distribution $\mathcal{G}_{n}(m)=$ Proba. $\left(l_{\min , n} \geq m\right)$ was studied in Ref. 22] and an explicit formula was obtained for its generating function:

$$
\sum_{n=0}^{\infty} \mathcal{G}_{n}(m) z^{n}=\frac{\sum_{l=m}^{\infty} Q(l) z^{l}}{1-\sum_{l=m}^{\infty} F(l) z^{l}},
$$

from which one gets the generating function of the average value $\left\langle l_{\min , \mathrm{n}}\right\rangle$ as

$$
\sum_{n=0}^{\infty} z^{n}\left\langle l_{\min , n}\right\rangle=\sum_{m=1}^{\infty} \frac{\sum_{l=m}^{\infty} Q(l) z^{l}}{1-\sum_{l=m}^{\infty} F(l) z^{l}} .
$$

In the absence of drift, $c=0$, it was shown in Ref. 22] that

$$
\left\langle l_{\min , n}\right\rangle \sim D \sqrt{n}, D=\frac{1}{\sqrt{\pi}} .
$$

As for $\left\langle l_{\max , n}\right\rangle$ we will see that the behavior of $\left\langle l_{\min , n}\right\rangle$, in the presence of non zero drift $c \neq 0$, is quite different in the five different regimes discussed above. Again we start by giving a brief summary of our results for $\left\langle l_{\min , n}\right\rangle$ :

$$
\begin{aligned}
& \left\langle l_{\min , n}\right\rangle \sim \sqrt{n} \text { for } 0<\mu<1 \text { and } c \text { arbitrary (regime I), } \\
& \sim n^{1-\theta(c)} \text { for } \quad \mu=1 \text { and } c \text { arbitrary (regime II), } \\
& \sim \text { const. for } 1<\mu<2 \text { and } c>0 \text { (regime III), } \\
& \sim \text { const. for } \mu=2 \text { and } c>0 \quad \text { (regime IV), } \\
& \sim n \text { for } 1<\mu \leq 2 \text { and } c<0 \text { (regime } \mathrm{V} \text { ), }
\end{aligned}
$$

again with $\theta(c)$ as defined in Eq. (16). In the following we discuss the behavior of $\left\langle l_{\min , n}\right\rangle$ in more detail for each of the five regimes.

6.2.1. Regime I: $0<\mu<1$ and $c$ arbitrary In this case the persistence probability decays algebraically as given in Eq. (143) and the analysis of $\left\langle l_{\min , n}\right\rangle$ can be obtained by noticing that, in the limit $z \rightarrow 1$, the denominator in Eq. (161) can be simply replaced 


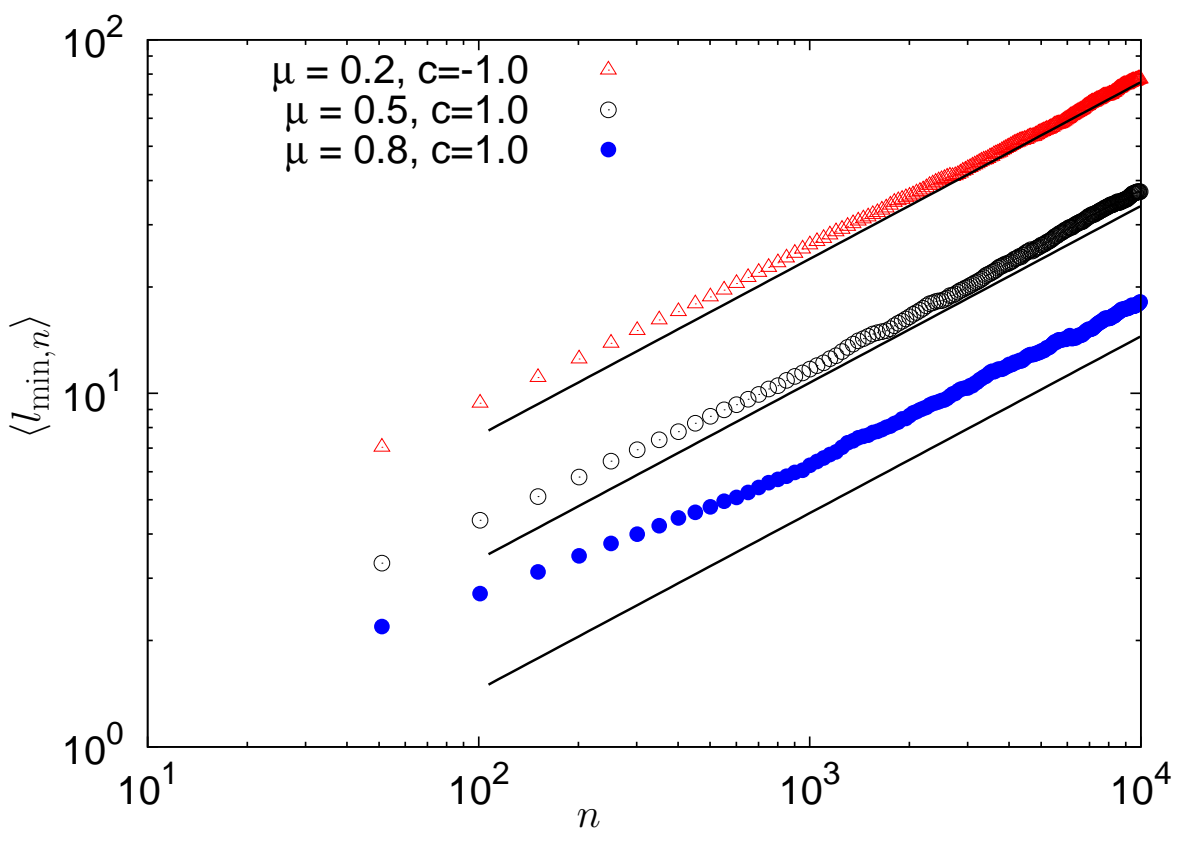

Figure 17. Plot, on $\log$-log scale, of $\left\langle l_{\min , n}\right\rangle$ as a function of $n$, for different values of $\mu<1$ and $c$ (regime I). The points are the results of numerical simulations while solid lines correspond to our exact analytic result given in Eq. (164). These data indicate that in this regime $\left\langle l_{\min , n}\right\rangle \propto \sqrt{n}$, although the corrections to the exact asymptotic behavior are clearly visible, in particular for $\mu=0.8, c=1.0$.

by 1 while the remaining sums over $l$ (in the numerator) and over $m$ can be replaced by integrals. This yields straightforwardly

$$
\begin{aligned}
& \left\langle l_{\text {min }, n}\right\rangle \sim D_{\mathrm{I}} \sqrt{n}, \\
& D_{\mathrm{I}}=B_{\mathrm{I}}=\frac{1}{\sqrt{\pi}} \exp \left[-\frac{1}{\pi} \int_{0}^{\infty} \frac{d k}{k} \arctan \left(\frac{\hat{f}(k) \sin (k c)}{1-\hat{f}(k) \cos (k c)}\right)\right],
\end{aligned}
$$

where the expression of $B_{\mathrm{I}}$ is given in Eq. (78). In Fig. 17, we show the results of our numerical simulations which are in a rather good agreement with Eq. (164), although the corrections to this exact asymptotic behavior are clearly visible, in particular for $\mu=0.8, c=1$.0. In Fig. 18, we show a plot of the numerical computation of $\left\langle l_{\min , n}\right\rangle$ for $\mu=1$ and different values of $c=-1,0.5$ and $c=1$ : these data are in good agreement with the power law growth in Eq. (164), although we have not attempted to estimate numerically the prefactor $D_{\mathrm{I}}$.

6.2.2. Regime II: $\mu=1$ and c arbitrary In this regime where the persistence probability $Q(m)$ decays algebraically as in Eq. (84), $\left\langle l_{\min , n}\right\rangle$ can be analyzed as in the regime I where in the limit $z \rightarrow 1$, the denominator in Eq. (161) can be simply replaced by 1 while the remaining sums over $l$ (in the numerator) and over $m$ can be replaced by 


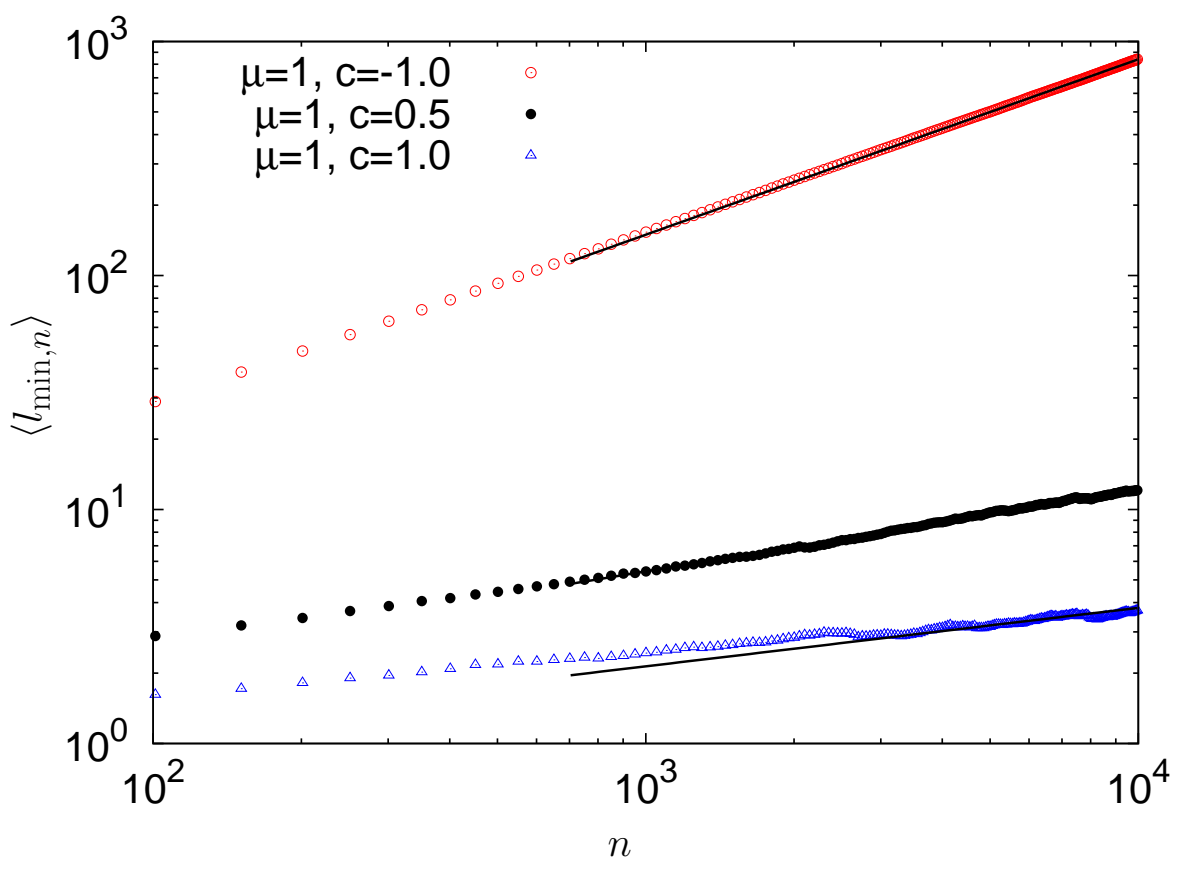

Figure 18. Plot, on a $\log -\log$ scale, of $\left\langle l_{\min , n}\right\rangle$ as a function of $n$ for $\mu=1$ and different values of $c=-1,0.5$ and $c=1$. The solid line corresponds to the algebraic growth $n^{1-\theta(c)}$, from Eq. (166).

integrals. This yields straightforwardly:

$$
\sum_{n=1}^{\infty} e^{-s n}\left\langle l_{\min , n}\right\rangle \sim \frac{B_{\mathrm{II}}}{s^{2-\theta(c)}} \int_{0}^{\infty} d y \int_{y}^{\infty} d x x^{-\theta(c)} e^{-y}=\frac{B_{\mathrm{II}}}{s^{2-\theta(c)}} \Gamma[2-\theta(c)],
$$

which yields

$$
\left\langle l_{\text {min }, n}\right\rangle \sim D_{\text {II }} n^{1-\theta(c)}, D_{\mathrm{II}}=B_{\mathrm{II}}
$$

where $B_{\mathrm{II}}$ is given in Eq. (85) and $\theta(c)=1 / 2+\frac{1}{\pi} \arctan (c)$.

6.2.3. Regime III: $1 \leq \mu<2$ and $c>0$ In this case we write the above formula (161) as

$$
\sum_{n=0}^{\infty} z^{n}\left\langle l_{\min , n}\right\rangle=\frac{1}{1-z}\left(1-\frac{1}{\tilde{q}(0)}\right)+\sum_{m=2}^{\infty} \frac{\sum_{l=m}^{\infty} Q(l) z^{l}}{1-\sum_{l=m}^{\infty} F(l) z^{l}},
$$

where we have simply isolated the term $m=1$ and used $1-\tilde{F}(0)=(1-z) \tilde{Q}(0)$ (58). Now the above sum (168), which starts with $m=2$, is dominated by the large values of $m$. Because of the algebraic decay of $Q(m) \sim m^{-\mu}$ in this case (148) and $\mu>1$ in this regime one gets that this second term behaves like $(1-z)^{\mu-2}$, which is then subleading, compared to the first term which behaves like $(1-z)^{-1}$. Therefore one gets in this case

$$
\left\langle l_{\mathrm{min}, n}\right\rangle \sim D_{\mathrm{III}}, D_{\mathrm{III}}=1-\frac{1}{\tilde{q}(0)}=1-\exp \left[-\sum_{n=1}^{\infty} \frac{1}{n} \int_{c n}^{\infty} P_{n}(x) d x\right],
$$




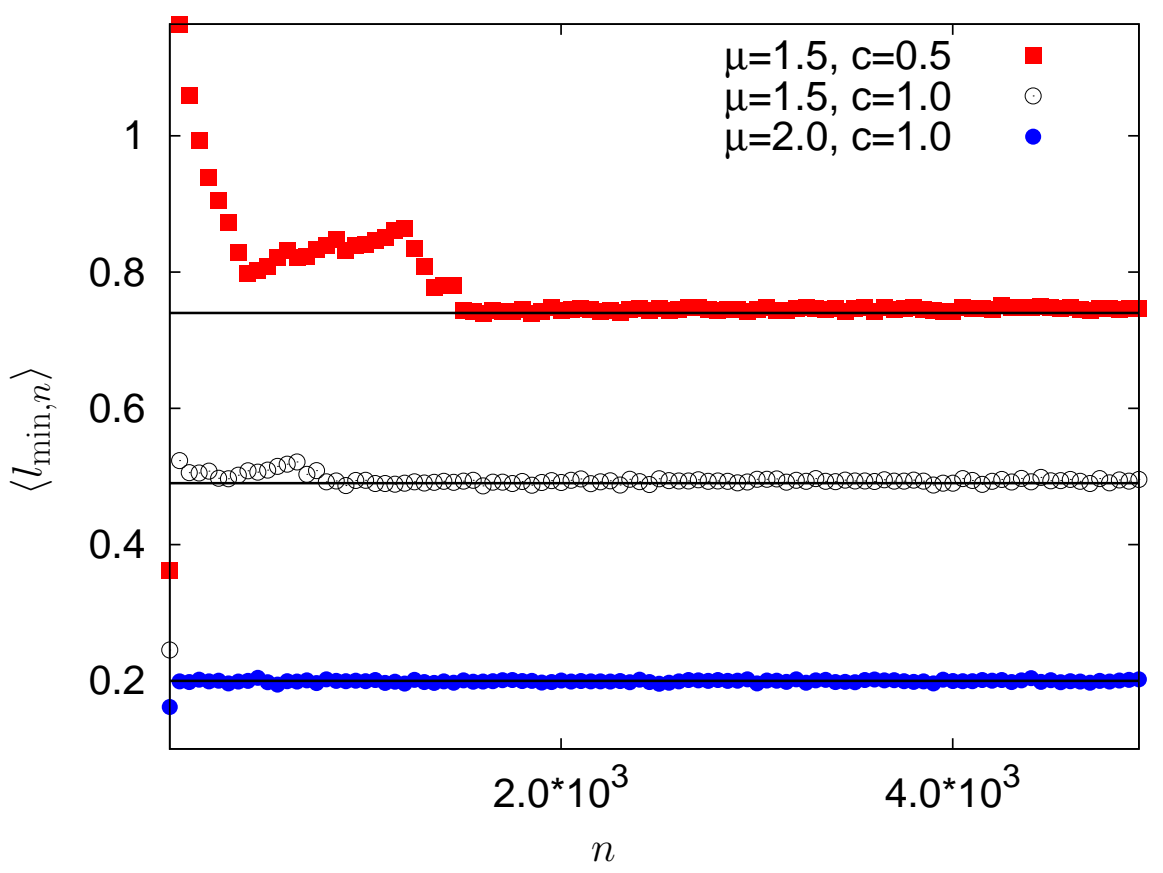

Figure 19. Plot of $\left\langle l_{\min , n}\right\rangle$ as a function of $n$ for $\mu=1.5$ and $\mu=2$ and different values of $c>0$, therefore corresponding to regime III and IV. The solid line corresponds to the exact result, from Eq. (169, 170).

where we have used the expression for $1 / \tilde{q}(0)$ given in Eq. (115). In Fig. 19 we show a plot of the numerical computation $\left\langle l_{\min , n}\right\rangle$ for $\mu=1.5$ and different values of $c=0.5$ and $c=1$, which is in very good agreement with Eq. (169). Note that we have extracted the value of $1 / \tilde{q}(0)$ which enters into the expression of $D_{\text {III }}$ from the linear growth of the mean record number $\left\langle R_{n}\right\rangle$, according to (115).

6.2.4. Regime $I V: \mu=2$ and $c>0$ A similar analysis can be carried out in this case, starting from the same formula (168). In this case, in the above sum (168), which starts with $m=2$, one can safely put $z=1$, because of the behavior of the exponential decay of $Q(m)$ in this case (151). Therefore one gets immediately

$$
\left\langle l_{\mathrm{min}, n}\right\rangle \sim D_{\mathrm{IV}}, D_{\mathrm{IV}}=1-\frac{1}{\tilde{q}(0)}=1-\exp \left[-\sum_{n=1}^{\infty} \frac{1}{n} \int_{c n}^{\infty} P_{n}(x) d x\right],
$$

where we have used the expression for $1 / \tilde{q}(0)$ given in Eq. (125). In Fig. 19 we show a plot of the numerical computation $\left\langle l_{\min , n}\right\rangle$ for $\mu=2$ and $c=1$, which is good agreement with Eq. (170). Note that we have extracted the value of $1 / \tilde{q}(0)$ which enters into the expression of $D_{\mathrm{IV}}$ from the linear growth of the mean record number $\left\langle R_{n}\right\rangle$, according to Eq. (125). 


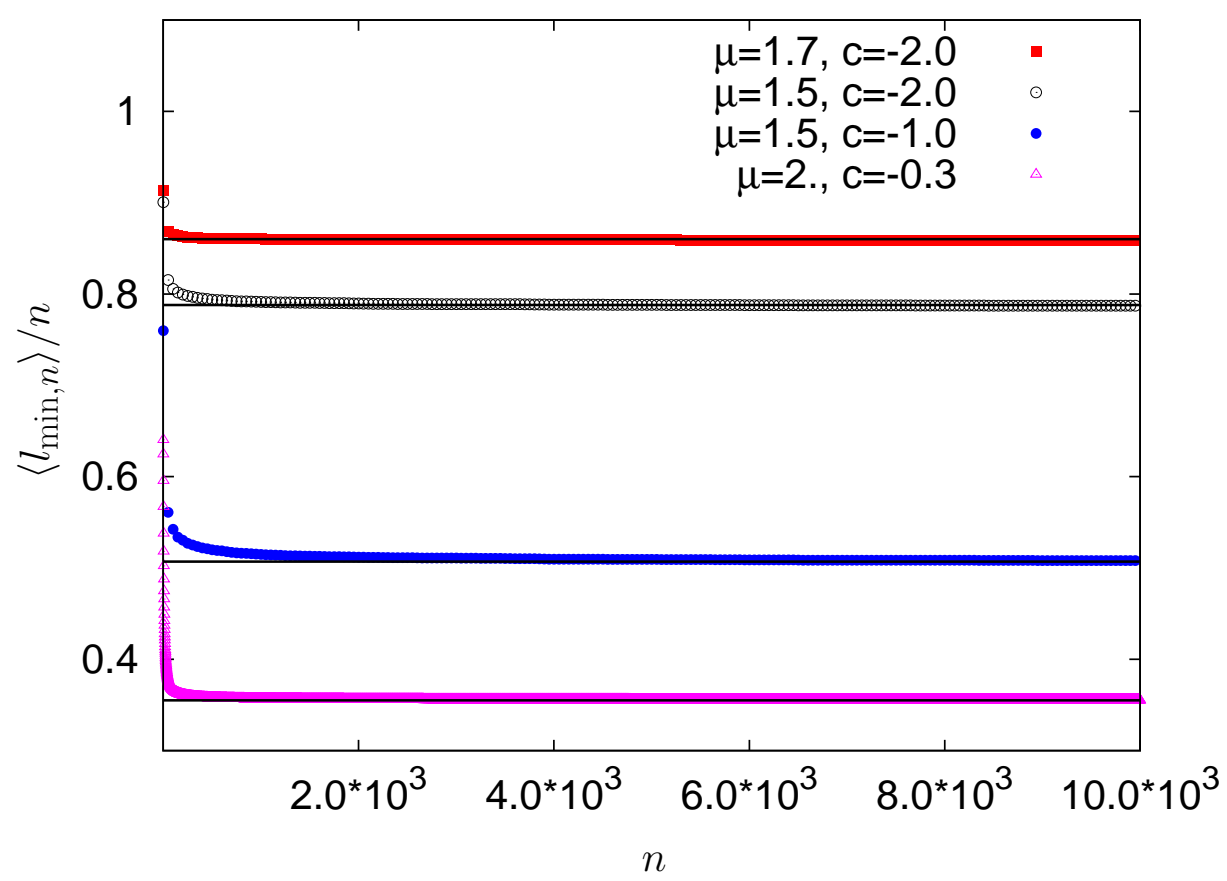

Figure 20. Plot of $\left\langle l_{\min , n}\right\rangle / n$ as a function of $n$ for different values of $1<\mu \leq 2$ and different values of $c<0$, corresponding to regime $\mathrm{V}$.

6.2.5. Regime $V: 1<\mu \leq 2$ and $c<0$ In this regime where the persistence goes to a constant $Q(m) \rightarrow \alpha_{\mu}(c)$, for $m \gg 1$, one can simply replace $Q(l)$ by this constant value in the sum of the numerator in Eq. (161) while the denominator can be simply approximated by 1 in the limit $1-z=e^{-s} \rightarrow 0$. This yields straightforwardly

$$
\left\langle l_{\min , n}\right\rangle \sim \alpha_{\mu}(c) n .
$$

In Fig. 20 we show a plot of $\left\langle l_{\min , n}\right\rangle / n$ which we have computed numerically for different values of $\mu=1.7,1.5$ and $\mu=2$ and also for different values of the drift. These results are in very good agreement with our exact asymptotic result in Eq. (171), where the value of $\alpha_{\mu}(c)$ have been extracted from the mean record number $\left\langle R_{n}\right\rangle \sim 1 / \alpha_{\mu}(c)$ (49). This result (171) can be easily understood by realizing that $l_{\min , n}=n$ if the whole trajectory is on the negative side, which happens with probability $\alpha_{\mu}(c)$ while $l_{\min , n}$ is of order $\mathcal{O}(1)$ if the walker makes an excursion on the positive side. One also notices that, in this case, $l_{\text {typ }} \sim\left\langle l_{\min , n}\right\rangle$.

\section{Conclusion}

In this paper we considered a very simple model of a one dimensional discrete-time random walk in presence of a constant drift $c$. At each time step the particle jumps by a random distance $c+\eta$ where the noise $\eta$ is drawn from a continuous and symmetric jump distribution $f(\eta)$, characterized by a Lévy index $0<\mu \leq 2$. The jump has a 


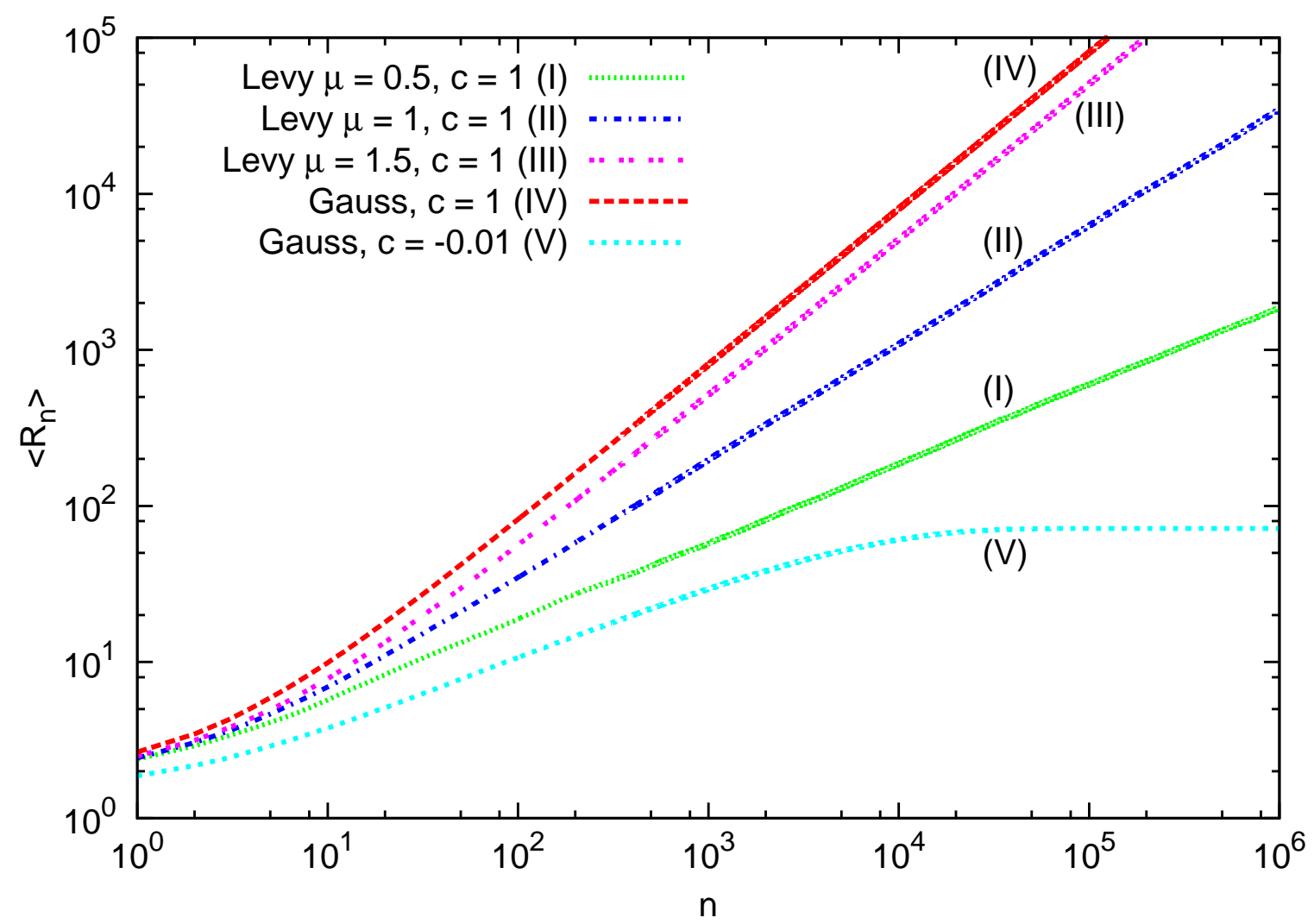

Figure 21. The figure shows numerical results for the mean record number $\left\langle R_{n}\right\rangle$ for biased random walks from all five regimes. For regimes I to IV we used a positive bias of $c=1$, in regime $\mathrm{V}$ we simulated a Gaussian random walk (with $\sigma=1$ ) with a negative bias of $c=-0.01$. For each jump distribution we averaged over $10^{4}$ samples. In all these cases, as shown in detail in the previous figures, the asymptotic behavior agree very well with our analytical predictions (which are not shown on this figure for clarity).

finite second moment for $\mu=2$, while for $0<\mu<2$ the second moment diverges. For this discrete-time series consisting of the successive positions of the biased walker, we presented complete analytical studies of the persistence and the record statistics. For the later, we studied the mean and the full distribution of the number of records up to step $n$ and also the statistics of the duration of records, in particular those for the longest and shortest lasting records. As a function of the two parameters $c$ and $0<\mu \leq 2$, we found that it is necessary to distinguish between five different universal regimes, as shown in the basic phase diagram in Fig. 1. In these 5 regimes, the persistence and the record statistics exhibit very different asymptotic behaviors that are summarized in Section 2 and we do not repeat them here. For instance, the growth of the mean record number with $n$ in all five regimes is summarized in the simulation results in Fig. 21, in complete agreement with our analytical predictions. The main conclusion is that even though this is a rather simple model, it exhibits very rich and varied universal behaviors for record statistics and persistence depending on the two parameters $c$ and $0<\mu \leq 2$.

Our results provide a simple yet nontrivial, but fully solvable model for the record 
statistics, a subject which has gained considerable interest over the last few years. Our results provide one generalization of the previous results for record statistics for symmetric random walks [22]. However, it is important to note that this extension does not yet cover all possible kinds of discrete-time random walks. In principle one could consider more complicated asymmetries of the jump distribution. It might be interesting to consider a jump distribution that has different tail-exponents in the left and in the right tail. Also a generalization of these results to an asymmetric lattice random walk is still missing. In [22] a symmetric lattice random was also considered. It should be possible to compute the record statistics of a lattice random walk that has a higher probability to jump in one direction than in the other.

It might be interesting to see if our results can be applied to financial data, similar to the analysis in [11, 12]. Daily stock data however proved not to be useful for comparison because the asymptotic limit is hardly achieved in the available observational data. An application to stock data with a higher temporal resolution however should be possible and might provide new insights. Such an analysis is definitely an interesting subject for future research. Also the distribution of records in stock prices has not been analysed in detail before and it would be interesting to see if such an analysis for available data can be fitted to our theoretical distributions.

Acknowledgments: SNM and GS acknowledge support by ANR grant 2011-BS04013-01 WALKMAT and in part by the Indo-French Centre for the Promotion of Advanced Research under Project 4604-3. GW is grateful for the kind hospitality of the Laboratoire de Physique Théorique et Modèles Statistiques during the completion of this work and for the financial support provided by DFG within the Bonn Cologne Graduate School of Physics and Astronomy.

\section{Appendix A. The constant $A_{\mathrm{I}}$}

The constant $A_{\mathrm{I}}$ in Eq. (104) can be directly expressed in terms of $\hat{f}(k)$ as announced in Eq. (24). To derive this, we use the explicit expression of $P_{n}(x)$ from Eq. (6) in the expression for $A_{\mathrm{I}}$ and integrate over $x$ to get

$$
A_{\mathrm{I}}=\frac{2}{\sqrt{\pi}} \exp \left[\sum_{n=1}^{\infty} \frac{1}{n} \int_{-\infty}^{\infty} \frac{d k}{2 \pi}[\tilde{f}(k)]^{n} \frac{1-e^{-i k c n}}{i k}\right] .
$$

Next we use the symmetry $\hat{f}(k)=\hat{f}(-k)$ which leads to

$$
A_{\mathrm{I}}=\frac{2}{\sqrt{\pi}} \exp \left[\frac{1}{\pi} \int_{0}^{\infty} \frac{d k}{k} \sum_{n=1}^{\infty} \frac{\sin (k c n)}{n}[\tilde{f}(k)]^{n}\right] .
$$

The sum on the rhs can be explicitly evaluated using the identity

$$
\sum_{n=1}^{\infty} \frac{x^{n}}{n} \sin (a n)=\arctan \left[\frac{x \sin (a)}{1-x \cos (a)}\right]
$$


which then leads to the exact expression in Eq. (24).

We then analyze the behavior of $A_{\mathrm{I}}$ when $|c|$ is large and in the case where $\hat{f}(k)=\exp \left(-|k|^{\mu}\right)$, with $\mu<1$. In that case one has $P_{n}(x)=n^{-1 / \mu} \mathcal{L}_{\mu}\left(x / n^{1 / \mu}\right)$ for all $n$ and it is easier to start from the formula given in the text in Eq. (104)

$$
A_{\mathrm{I}}=\frac{2}{\sqrt{\pi}} e^{S_{0}}, S_{0} \equiv S_{0}(c)=\sum_{n=1}^{\infty} \frac{1}{n} \int_{0}^{c n} \mathcal{L}_{\mu}\left(x / n^{1^{1 / \mu}}\right) d x / n^{1 / \mu} .
$$

Note that, given that $P_{n}(x)=P_{n}(-x)$ one has $S_{0}(c)=S_{0}(-c)$ and we thus present the analysis for $c>0$. Performing the change of variable $y=x / n^{1 / \mu}$ in the integral above (A.4) we write

$$
S_{0}(c)=\sum_{n=1}^{\infty} \frac{1}{n} \int_{0}^{c n^{\frac{\mu-1}{\mu}}} \mathcal{L}_{\mu}(y) d y,
$$

and take the derivative with respect to $c$

$$
S_{0}^{\prime}(c)=\sum_{n=1}^{\infty} n^{-\frac{1}{\mu}} \mathcal{L}_{\mu}\left(\frac{c}{n^{\frac{1-\mu}{\mu}}}\right) .
$$

In this expression, one notices that $c / n^{\frac{1-\mu}{\mu}}=\left(n / c^{\frac{\mu}{1-\mu}}\right)^{\frac{\mu-1}{\mu}}$ so that when $c \rightarrow \infty$ the discrete sum over $n$ in Eq. (A.6) can be replaced by an integral (we recall that $\mu<1$ here), which leads to

$$
S_{0}^{\prime}(c) \sim \frac{1}{c} \int_{0}^{\infty} \mathcal{L}_{\mu}\left(y^{\frac{\mu-1}{\mu}}\right) y^{-1 / \mu} d y .
$$

Finally, performing the change of variable $z=y^{\frac{\mu-1}{\mu}}$ in Eq. (A.7) yields

$$
S_{0}^{\prime}(c) \sim \frac{1}{c} \frac{\mu}{1-\mu} \int_{0}^{\infty} \mathcal{L}_{\mu}(z) d z=\frac{1}{c} \frac{\mu}{2(1-\mu)},
$$

so that one gets

$$
A_{\mathrm{I}}=\frac{2}{\sqrt{\pi}} e^{S_{0}} \propto c^{\frac{\mu}{2(1-\mu)}}, c \rightarrow \infty .
$$

This power law behavior (A.9) can be understood from the following scaling argument. We are indeed interested in the records statistics of the variables $y_{n}$, with $y_{n}=$ $x_{n}+c n$ (11) where $x_{n}$ behaves for large $n$ as $x_{n}=\mathcal{O}\left(n^{1 / \mu}\right)$. Therefore for small $n, n<n^{*}$ when $c$ is large, $y_{n}$ is dominated by the drift term and $n^{*}$ is such that $c n^{*} \sim n^{* 1 / \mu}$, which yields

$$
n^{*} \sim c^{\frac{\mu}{1-\mu}} .
$$

On the other hand, for small $n, n<n^{*}, y_{n}$ is dominated by the (positive) drift and hence is almost deterministic which yields $\left\langle R_{n}\right\rangle \sim n$, for $n<n^{*}$ while $\left\langle R_{n}\right\rangle \sim A_{\mathrm{I}} \sqrt{n}$ for $n>n^{*}$. By matching these two behaviors for $n=n^{*}$ one obtains

$$
A_{\mathrm{I}} \sim \sqrt{n^{*}} \propto c^{\frac{\mu}{2(1-\mu)}},
$$

which yields the result obtained above (A.7).

Note finally that, by using $S_{0}(c)=-S_{0}(-c)$ one obtains

$$
A_{\mathrm{I}} \sim(-c)^{\frac{-\mu}{2(1-\mu)}}, c \rightarrow-\infty .
$$




\section{Appendix B. Computation of $\alpha_{2}(c)=a_{2}(|c|), c<0$ for exponential jump distribution with $c<0$}

The expression for the amplitude $\alpha_{2}(c)$ in regime $\mathrm{V}$ (with $c<0$ ) and for a general jump distribution is given in Eq. (101). By comparing with Eq. (125) we see that $\alpha_{2}(c<0)=a_{2}(|c|)$ where $a_{2}(|c|)$ is the prefactor of the leading linear growth of mean record number in regime IV with drift positive $|c|$. For a general jump distribution $f(\eta)$, we then have

$$
\alpha_{2}(c)=\exp \left[-\sum_{n=1}^{\infty} \frac{1}{n} \int_{|c| n}^{\infty} P_{n}(x) d x\right],
$$

where we recall that $P_{n}(x)=\int_{-\infty}^{\infty} \frac{d k}{2 \pi}[\hat{f}(k)]^{n} e^{-i k x}$ and $\hat{f}(k)=\int_{-\infty}^{\infty} f(\eta) e^{i k \eta} d \eta$ is the Fourier transform of the jump distribution. Thus, in general, computing the prefactor $\alpha_{2}(c)=a_{2}(|c|)$ explicitly is difficult for arbitrary $f(\eta)$. It can be done explicitly for Gaussian distribution where $P_{n}(x)=\left(2 \pi n \sigma^{2}\right)^{-1 / 2} \exp \left[-x^{2} / 2 n \sigma^{2}\right]$ itself is Gaussian and $\alpha_{2}(c)=a_{2}(|c|)$ is then given by the formula in Eq. (126). In this appendix, we show that $\alpha_{2}(c)=a_{2}(|c|)$ can also be computed explicitly for the symmetric exponential distribution $f(\eta)=(2 b)^{-1} \exp (-|x| / b)$.

For this exponential jump distribution, the Fourier transform has the Lorentzian form, $\hat{f}(k)=1 /\left[\pi\left(b^{2} k^{2}+1\right)\right]$. One can then substitute this in the expression for $P_{n}(x)$ and eventually in Eq. (B.1). After a quite convoluted computation involving contour integration in the complex plane, one can find $\alpha_{2}(c)$ explicitly. However, as we show below, for the exponential case, there is an alternative simpler way to compute $\alpha_{2}(c)$ directly (without going through the formula in Eq. (B.1).

The first observation is that $\alpha_{2}(c)$ is just the limiting value of the persistence probability $Q(n)$ (the probability that the walker stays below 0 up to $n$ steps starting at 0 ) when $n \rightarrow \infty$ in presence of a negative drift $c<0$. By symmetry, $Q(n)$ is then also the probability that the walker, starting at the origin, stays above the origin up to $n$ steps, but in presence of a positive drift $|c|>0$. So, the idea is to compute this probability $Q(n)$ directly for the exponential jump distribution and then take the limit $n \rightarrow \infty$ to compute $\alpha_{2}(c)=Q(n \rightarrow \infty)$.

To compute $Q(n)$, we first define

$q_{n}^{+}(y)=$ Proba. that the random walker, starting at $y \geq 0$ stays positive up to step $n$.

If we can compute $q_{n}^{+}(y)$, then $Q(n)$ is simply obtained by putting the starting position to be 0 , i.e., $Q(n)=q_{n}^{+}(0)$. To compute $q_{n}^{+}(y)$, we can write a backward recurrence relation for $q_{n}^{+}(y)$ by considering the jump that happens at the first step from $y$ to $y^{\prime} \geq 0$

$$
\begin{aligned}
& q_{n}^{+}(y)=\int_{0}^{\infty} q_{n-1}^{+}\left(y^{\prime}\right) f\left(y+|c|-y^{\prime}\right) d y^{\prime}, \\
& q_{0}^{+}(y)=1 \text { for } y \geq 0 .
\end{aligned}
$$


In the limit of large $n$, we expect that $q_{n}^{+}(y)$ approaches to an $n$ independent stationary value, $q_{n}^{+}(y) \rightarrow q^{+}(y)$, that just denotes the eventual probability with which the walker escapes to infinity (starting from $y$ ) in presence of a positive drift $|c|$. Taking $n \rightarrow \infty$ limit on both sides of Eq. (B.3) gives the integral equation for $y \geq 0$

$$
q^{+}(y)=\int_{0}^{\infty} q^{+}\left(y^{\prime}\right) f\left(y+|c|-y^{\prime}\right) d y^{\prime}
$$

Note that this equation is valid for arbitrary jump distribution $f(\eta)$. This half-space Wiener-Hopf type integral equation with asymmetric kernel can not be solved in general. However, for the special case of the exponential distribution, $f(\eta)=1 /(2 b) \exp (-|\eta| / b)$, this integral equation (B.5) can be transformed into a differential equation using

$$
f^{\prime \prime}(\eta)=-\frac{1}{b^{2}} \delta(\eta)+\frac{1}{b^{2}} f(\eta) .
$$

By differentiating twice Eq. (B.5) with respect to $y$ one then obtains [using Eq. (B.6)]

$$
\frac{d^{2} q^{+}(y)}{d y^{2}}=-\frac{1}{b^{2}} q^{+}(y+|c|)+\frac{1}{b^{2}} q^{+}(y) \text {. }
$$

Note that the solution $q^{+}(y)$ must approach to 1 as $y \rightarrow \infty: q^{+}(y \rightarrow \infty)=1$. This follows from the fact that if the particle starts at the positive infinity, it escapes to positive infinity with probability 1 in presence of any positive drift $|c|>0$.

Note that the differential equation (B.7), though linear, is actually nonlocal in $y$ due to the first term on the rhs and hence is still not completely trivial to solve. Fortunately, it turns out that it admits a solution of the form

$$
q^{+}(y)=1-F \exp (-\lambda y / b),
$$

where $F$ and $\lambda$ are two dimensionless constants (independent of $y$ ) that are yet to be determined. Note that this ansatz manifestly satisfies the boundary condition $q^{+}(y \rightarrow \infty)=1$. Substituting this ansatz in Eq. (B.7) we see that indeed Eq. (B.8) is a solution provided $\lambda$ satisfies the equation

$$
\exp (-\lambda|c| / b)=1-\lambda^{2} ; \text { with } \lambda>0 .
$$

The transcendental equation has a unique positive solution which then determines $\lambda$ uniquely. For example, for $b / c=1$, we get using Mathematica the root $\lambda=0.714556 \ldots$ But we still need to determine the prefactor $F$ in the ansatz in Eq. (B.8). The amplitude $F$ in Eq. (B.8) is obtained by injecting this solution back into the integral equation (B.7) and performing the integral. Indeed, one finds that Eq. (B.8) is a solution of the integral equation provided

$$
F=1-\lambda .
$$

This then uniquely determines the solution of the integral equation (B.7)

$$
q^{+}(y)=1-(1-\lambda) \exp (-\lambda y / b)
$$

where $\lambda$ is the unique positive solution of the transcendental equation (B.9). 
Noting finally that $\alpha_{2}(c)=Q(n \rightarrow \infty)=q^{+}(0)$ gives

$$
\alpha_{2}(c)=a_{2}(|c|)=q^{+}(0)=\lambda \text {, }
$$

where $\lambda>0$ is the solution of Eq. (B.9). We have checked that we indeed get exactly the same expression by evaluating the original general expression in Eq. (B.1) for the

exponential jump distribution, though this was not completely trivial to check (we do not give details of this check here).

\section{References}

[1] D. Gembris, J.G. Taylor, and D. Suter, Nature 417506 (2002).

[2] D. Gembris, J.G. Taylor, and D. Suter, J. Appl. Stat. 34529 (2007).

[3] R. Redner and M.R. Petersen, Phys. Rev. E 74061114 (2006).

[4] G.A. Meehl, C. Tebaldi, G. Walton, D. Easterling, and L. McDaniel, Geophys. Res. Lett. 36 L23701 (2009).

[5] G. Wergen and J. Krug, Europhys. Lett. 92, 30008 (2010).

[6] A. Anderson and A. Kostinski, J. Appl. Meteo. and Climat. 50, 1859 (2011).

[7] J. Krug and K. Jain, Physica A 358, 1 (2005).

[8] L.P. Oliveira, H.J. Jensen, M. Nicodemi, and P. Sibani, Phys. Rev. B 71, 104526 (2005).

[9] P. Sibani P, G.F. Rodriguez, and G.G. Kenning, Phys. Rev. B 74, 224407 (2006).

[10] C. Godrèche and J. M. Luck, J. Stat. Mech. P11006 (2008).

[11] G. Wergen, M. Bogner, and J. Krug, Phys. Rev. E 83, 051109 (2011).

[12] G. Wergen, S. N. Majumdar, and G. Schehr, Phys. Rev. E 86, 011119 (2012).

[13] F. G. Foster and A. Stuart, J. Roy. Stat. Soc. 16, 1 (1954).

[14] B. C. Arnold, N. Balakrishnan, and H. N. Nagaraja, Records, Wiley (1998).

[15] V. B. Nevzorov, Records: Mathematical Theory, Am. Math. Soc. (2001).

[16] R. Ballerini and S. Resnick, J. Appl. Prob. 22, 487 (1985).

[17] J. Franke, G. Wergen, and J. Krug, J. Stat. Mech. P10013 (2010).

[18] G. Wergen, J. Franke, and J. Krug, J. Stat. Phys. 144, 1206 (2011).

[19] J. Franke, G. Wergen, and J. Krug, Phys. Rev. Lett. 108, 064101 (2012).

[20] J. Krug, J. Stat. Mech. P07001 (2007).

[21] G.H. Weiss, Aspects and applications of the random walk, (North-Holland, 1994).

[22] S. N. Majumdar and R. M. Ziff, Phys. Rev. Lett. 101, 050601 (2008).

[23] E. Sparre Andersen, Matematica Scandinavica, 2: 195-223 (1954).

[24] S. N. Majumdar, Physica A 389, 4299 (2010) (lecture notes for the summer school "Fundamental Problems in Statistical Physics: XII" held at Leuven, Belgium (2009)).

[25] S. Sabhapandit, Europhys. Lett. 94, 20003 (2011).

[26] P. Le Doussal and K. J. Wiese, Phys. Rev. E 79, 051105 (2009).

[27] Y. Edery, A. Kostinski, and B. Berkowitz, Geophys. Res. Lett. 38, L16403 (2011).

[28] S. N. Majumdar, Curr. Sci. 77, 370 (1999).

[29] J.-P. Bouchaud and A. Georges, Phys. Rep. 195, 127 (1990).

[30] R. Metzler and J. Klafter, Phys. Rep. 339, 1 (2000).

[31] M. R. Evans, S. N. Majumdar, R. K. P. Zia, J. Stat. Phys. 123, 357 (2006).

[32] G. Schehr, P. Le Doussal, J. Stat. Mech. P01009 (2010).

[33] J. Pitman, M. Yor, Ann. Probab. 25, 855 (1997).

[34] C. Godrèche, S. N. Majumdar, G. Schehr, Phys. Rev. Lett. 102, 240602 (2009). 